Article

\title{
Petrographical and Geochemical Characteristics of Magmatic Rocks in the Northwestern Siberian Traps Province, Kulyumber River Valley. Part II: Rocks of the Kulyumber Site
}

\author{
Nadezhda Krivolutskaya ${ }^{1, *(\mathbb{D})}$, Boris Belyatsky ${ }^{2}{ }^{-}$, Bronislav Gongalsky $^{3}$, Alexander Dolgal ${ }^{4}$, \\ Andrey Lapkovsky ${ }^{5}$ and Tamara B. Bayanova ${ }^{6}$ \\ 1 Vernadsky Institute of Geochemistry and Analytical Chemistry, Russian Academy of Sciences, \\ Kosygin st. 19, 119991 Moscow, Russia \\ 2 A.P. Karpinsky Russian Geological Research Institute, Sredny Prospect, 74, 199106 St. Petersburg, Russia; \\ bbelyatsky@mail.ru \\ 3 Institute Geology of Ore Deposits, Petrography, Mineralogy and Geochemistry, Russian Academy of \\ Sciences, Staromonetny per., 35, 119109 Moscow, Russia; brgon@mail.ru \\ 4 Geological Department, Perm State University, 15 Bukireva, 614990 Perm, Russia; Dolgal@mi-perm.ru \\ 5 Geological Institute of Kola Scientific Center, Russian Academy of Sciences, Fersman st., 14, \\ 184209 Apatity, Russia; alapkovsky@gmail.com \\ 6 Norilskgeology Ltd., Grazhdansky pr., 11, 195220 St. Petersburg, Russia; tamara@geoksc.apatity.ru \\ * Correspondence: nakriv@mail.ru; Tel.:+7-926-543-4787
}

Received: 3 April 2020; Accepted: 3 May 2020; Published: 7 May 2020

\begin{abstract}
The origin of the Siberian trap province is under discussion even though numerous models of its formation have been created over the last three decades. This situation is mainly due to lack of modern geochemical data on magmatic rocks around the province. These data are a very important tool to reconstruct of magmatic evolution within the province in time and space and to understand a mechanism of province formation. Geochemical study has only been carried out so far for the Norilsk and Meimecha-Kotuy areas. For the first time, we have studied the geochemical and mineralogical characteristics of magmatic rocks at the Kulyumber river valley located $150 \mathrm{~km}$ to south from the Norilsk ore district, in the junction of the Tunguska syneclise and Norilsk-Igarka zone. It comprises three sites, i.e., Khalil, Kaya, and Kulyumber. The geochemical data on the magmatic rocks of the Khalil and Kaya sites were published earlier (Part I). This article (Part II) regards geochemical and mineralogical data on igneous rocks at the Kulyumber site. Seventeen intrusive bodies (41 samples) and six samples of sedimentary rocks were studied by X-ray fluorescence (XRF) and inductively coupled plasma mass spectrometry (ICP-MS). Isotopes analyses $(\mathrm{Sr}, \mathrm{Nd}, \mathrm{Pb})$ were conducted for 12 samples. These data were compared with data for intrusions of the Norilsk area, the Dzhaltulsky massif, Kureyka river, and intrusions in Angara river valley published earlier. The whole list of analyses includes 102 items. Three groups of intrusive rocks were recognized: (1) Mafic rocks with elevated $\mathrm{K}_{2} \mathrm{O}$ without negative Ta- $\mathrm{Nb}$ and $\mathrm{Pb}$-positive anomalies, with $(\mathrm{Gd} / \mathrm{Yb}) \mathrm{n}=2.0$ and $\varepsilon \mathrm{Nd}=-1.0$; attributed to a new Kulyumbinsky complex; (2) subalkaline rocks with elevated $\mathrm{SiO}_{2}, \mathrm{TiO}_{2}, \mathrm{P}_{2} \mathrm{O}_{5}$, and $\mathrm{K}_{2} \mathrm{O}$ with small negative $\mathrm{Ta}-\mathrm{Nb}$ and positive $\mathrm{Pb}$ anomalies and $(\mathrm{Gd} / \mathrm{Yb}) \mathrm{n}=1.8$, $\varepsilon \mathrm{Nd}=-3.8$; Ergalakhsky complex; and (3) mafic rocks with strong $\mathrm{Ta}-\mathrm{Nb}$ and $\mathrm{Pb}$ anomalies and $(\mathrm{Gd} / \mathrm{Yb}) \mathrm{n}=1.2-1.4, \varepsilon \mathrm{Nd}=+0.4-+2.2$. The third group is rather nonhomogeneous and includes intrusions of the Norilsk, Kuryesky, Katangsky, Ogonersky, and Daldykansky complexes differing in $\mathrm{MgO}$ content and trace element distribution (values of $\mathrm{Ta}-\mathrm{Nb}, \mathrm{Pb}$, and $\mathrm{Sr}$ anomalies). Three groups of intrusive bodies had different magma sources and different condition of crystallization reflecting their origin in rift and platform regimes.
\end{abstract}


Keywords: Siberian traps; Norilsk-Igarka paleorift; Kulyumber river valley; magmatism; geochemistry

\section{Introduction}

The origin of the Siberian traps province, the largest continental flood basalt province on Earth (SFBP), is still under discussion, even though it has been studies for many years [1-17]. A huge volume of magmatic rocks and related extra-large PGE-Cu-Ni deposits are the main points of interest for geologists [18-29]. Many different models have been suggested for its formation and the source of mineralization [5,21,30-32]. However, none of these models satisfies the requirements for the quality of modern constraints. The main problem arises from the lack of geological data since the SFBP is vast and comprises many remote and inaccessible areas. Nevertheless, advances in the use of geochemical data can help to improve our understanding of magmatic rocks around the Siberian province and also to reconstruct the history of their formation and evolution. By this moment, precious geochemical data were obtained for two regions of the province, Norilsk and Meimecha-Kotuy [33-44]. These data are mostly from basaltic rocks. Investigation of intrusive rocks comprised mainly the Norilsk ore-bearing intrusions [34,35,45-50], while the intrusions from other parts of the province have almost not been analyzed yet.

The research of igneous rocks around the Siberian province is an ongoing project and the results will be published in a series of articles. The first area that was studied was the Kulyumber river valley (KRV) located $150 \mathrm{~km}$ to the south of the Norilsk area [50] (Figure 1). Its tectonic structures and magmatic rocks are very similar to that of the unique Norilsk ore district. The KRV is located between the Tunguska syneclise and Norilsk-Igarka paleorift tectonic zone and provide information on magmatic activities within both structures. Additionally, unlike poorly mineralized nearby areas, the KRV includes some disseminated and massive sulfide ore potentials. We studied igneous rocks at three sites within the Kulyumber river valley including two northern sites (Khalil and Kaya) and one southern site, Kulyumber site.

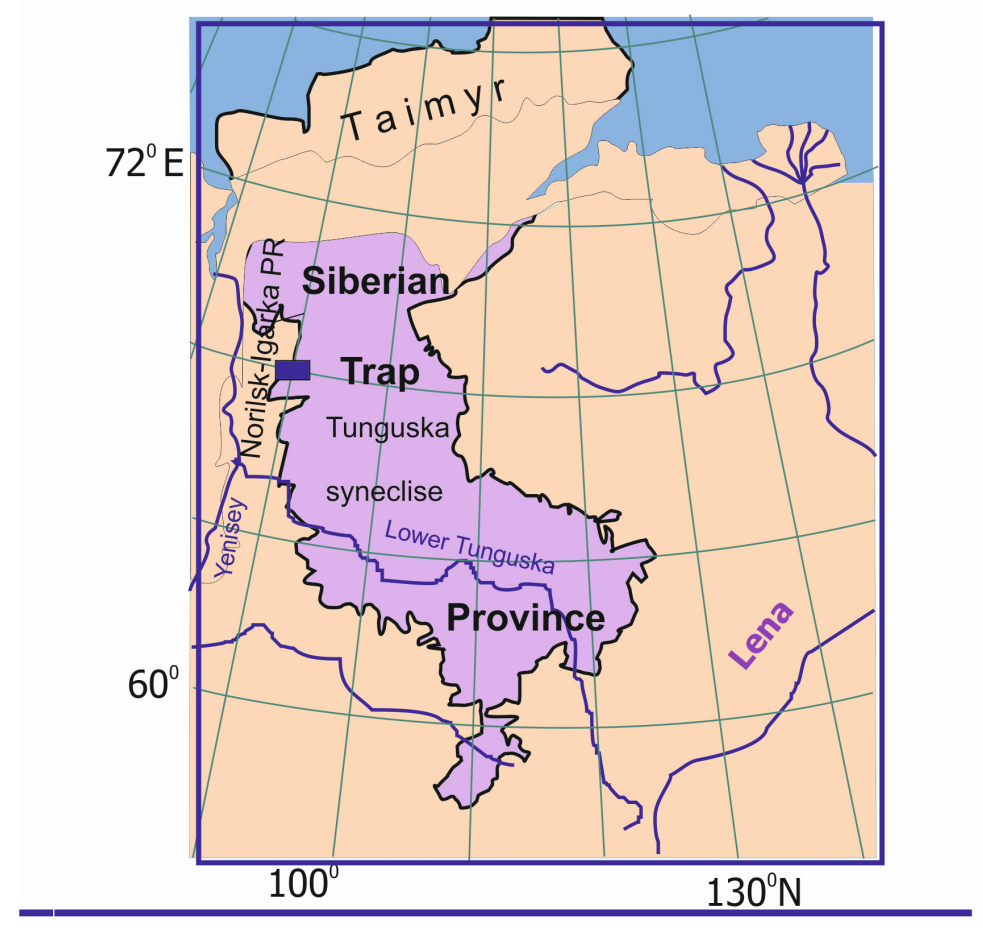

Figure 1. Scheme of the Siberian traps province with position of the Kulyumber river valley (dark rectangle). Norilsk-Igarka PR—paleorift zone. 
This article briefly describes the geology of the KRV and petrological and geochemical characteristics of rocks (mostly, intrusive rocks) of the Kulyumber site, whereas, magmatic rocks in the northern part of this area were described earlier [51]. For the first time, the authors gathered a large amount of modern geochemical and mineralogical data on magmatic rocks of the Kulyumber river valley. That allowed us to subdivide intrusive rocks into complexes more correctly than was done before.

\section{Brief Geological Characteristics of the Kulyumber River Valley}

The study area is located in the Northwestern Siberian platform (Figure 1) where the Tunguska syneclise joins with the Norilsk-Igarka paleorift. The tectonic structure of the area is described in [51] on the basis of geophysical data (1:200,000 gravity mapping and aeromagnetic data of 1:100,000 scale). It is characterized by a submeridional band of the gravitational field positive values and low values of the magnetic field stretching along the Yenisey river (100 km wide) and bordering the ancient Siberian craton. It has three sublatitude branches, one of them coincides with the stretch of the Kulyumber river valley.

The local tectonic structures include the Nirungdinsky trough and the Mogen-Khalil anticline (Figure 2). The Lower Ordovician-Lower Carboniferous formations in the area consist of carbonate-terrigenous rocks, whereas the Tunguska Group $\left(\mathrm{C}_{2}-\mathrm{P}_{2}\right)$ sediments are composed of sandstones and coal. The Late Permian-Early Triassic magmatic rocks belong to the Siberian flood basalts province. Like the Norilsk area [52], volcanic rocks from the Nirungdinsky trough are divided into several formations, including Syverminsky, Gudchikhinsky, Khakanchansky, Tuklonsky, and Nadezhdinsky, that were comprehensively described in the first part of the article [51]. The two lower formations contain trachybasalts and olivine basalts with $\mathrm{TiO}_{2}$ content $>1.5 \mathrm{wt} . \%$ and high $\mathrm{Gd} / \mathrm{Yb}$ ratio, which evidences presence of garnet in magmas' sources. The upper formations consist of tholeiitic basalts with $\mathrm{TiO}_{2}<1$ wt. $\%$ and low $\mathrm{Gd} / \mathrm{Yb}$ ratio.

Intrusive bodies are mostly enclosed by sedimentary rocks, and are exposed in the western part of the area (Figure 1). During the geological mapping (1:200,000 scale) these rocks were classified into several complexes on the basis of their textures and structure as well as a few X-ray fluorescence (XRF) analyses. They were attributed to the intrusive complexes typical of the Tunguska syneclise (Katangsky, Kuzmovsky, [53]), the Norilsk area (Ergalakhsky, Norilsk, Daldykansky, Ogonersky) [52], and the local Kureysky complex. However, this classification does not take into account modern geochemical techniques that allow distinguishing different types of intrusive rocks more precisely.

The intrusions occur as sill-like bodies (with a thickness up to 5-6 to $100 \mathrm{~m}$ and a length of up to 10-15 km). Rare dykes cut volcanic rocks. The magma intrusion was mainly controlled by Imangdinsky-Letninsky and Khalilsky faults (Figure 2). Intrusive bodies compositionally vary from olivine gabbro-dolerite to gabbro and leucogabbro. Magnesium-rich rocks ( $\mathrm{MgO}=9$ wt. $\%)$ rather occur as separate horizons within differentiated intrusions than forming separate bodies. 


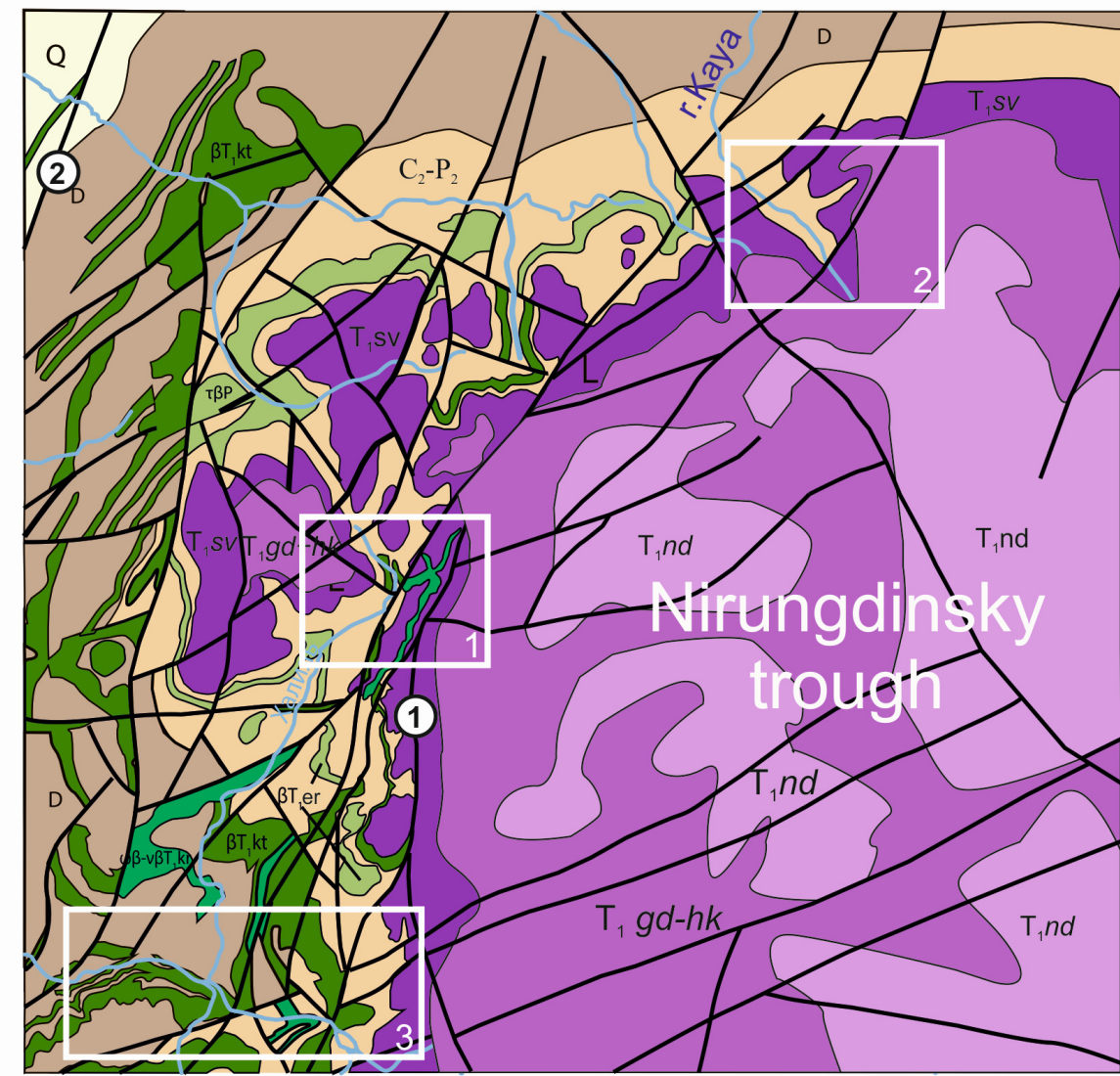

$$
\mathrm{m} 2500 \quad 0 \quad 2500 \quad 5000 \mathrm{~m}
$$

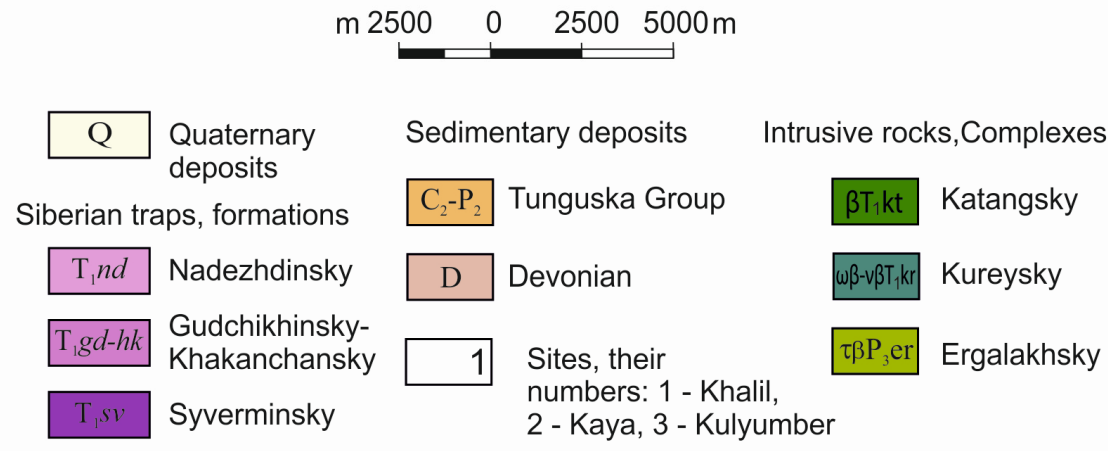

(1) Faults and their numbers: 1 - Khalil, 2 - Mogen

Figure 2. Geological map of the Kulyumber river valley (after Norilskgeology Ltd. data with the authors' corrections).

\section{Materials and Methods}

The authors studied igneous rocks sampled from the outcrops in the Kulyumber river valley including three sites, i.e., the (1) Khalil, (2) Kaya, and (3) Kulyumber (samples labeled X, 3.21-3.22, and Kul, respectively) (Figures 2 and 3 [51]) and from the cores of the boreholes PR-1, PR-4, and PR-11 drilled by Norilskgeology Ltd. and shown in Figure 4. The last area is located immediately to the west from the Kulyumber site. Its eastern boundary coincides with the western boundary of the area shown on Figure 3. We named it the western Kulyumber site. The data on magmatic rocks from two first areas were considered in Part I of the article [51]. Some samples of Devonian and Carboniferous-Permian sedimentary rocks were analyzed as well. In order to compare the magmatic rocks with the rocks of the Norilsk district, we also studied gabbro-dolerites of the Daldykansky and 
Ogonersky complexes (outcrops). Furthermore, we used geochemical data published by us earlier [51] for the ore-bearing Norilsk 1 intrusion (borehole DM-27), the Dzhaltulsky massif in the Kureyka river valley, and Padunsky sill in the Angara river valley, regarded as reference intrusions for the Norilsk, Kureysky, and Katangsky complexes, respectively.

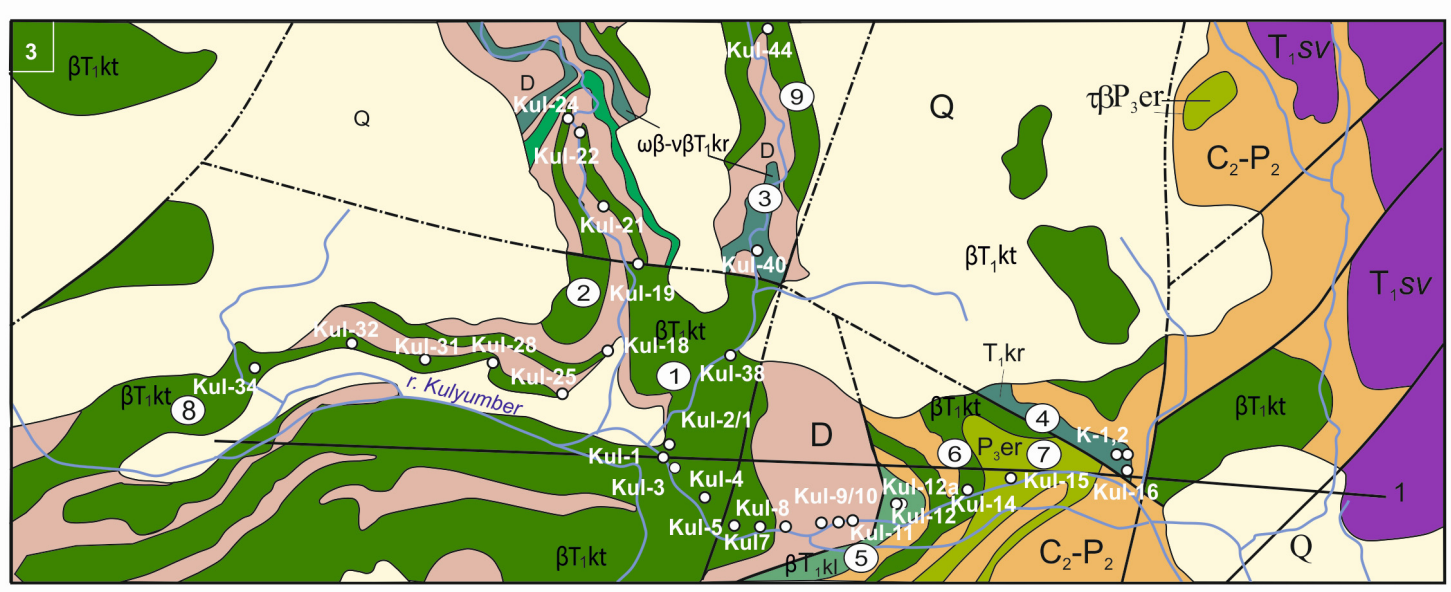

m $500 \quad 0 \quad 50010001500$ m

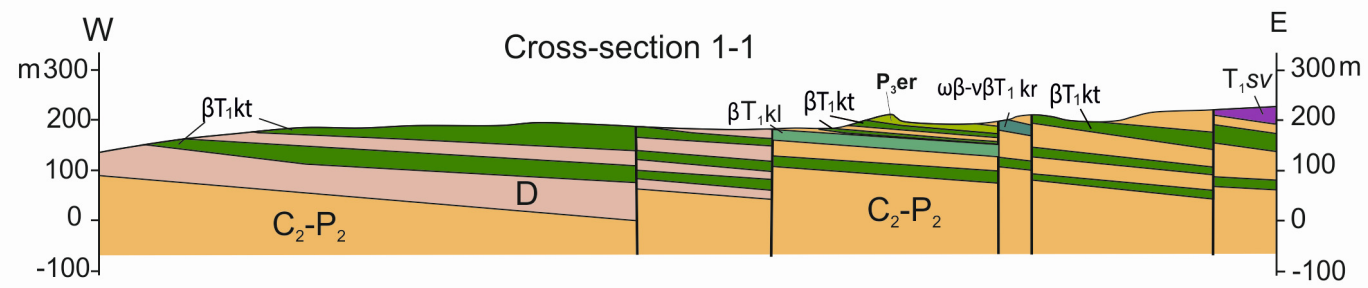

Q Quaternary

Sedimentary deposits

Intrusive rocks, Complexes

Siberian traps, formation

$\mathrm{C}_{2}-\mathrm{P}_{2}$ Tunguska Group

$\beta \mathrm{T}_{1} \mathrm{kt}$ Katangsky

$\omega \beta-v \beta T$ T kr Kureysky

Samples and

$T_{,} s v$ Syverminsky

D Devonian

$\beta \mathrm{T}_{1} \mathrm{kl}$ Kulyumbinsky

$\tau \beta \mathrm{P}_{3} \mathrm{er}$ Ergalakhsky

their numbers

Figure 3. Geological map and cross-section of the of the Kulyumber site. The number in circle means the intrusion number (after Norilskgeology Ltd. data with the authors' corrections). 


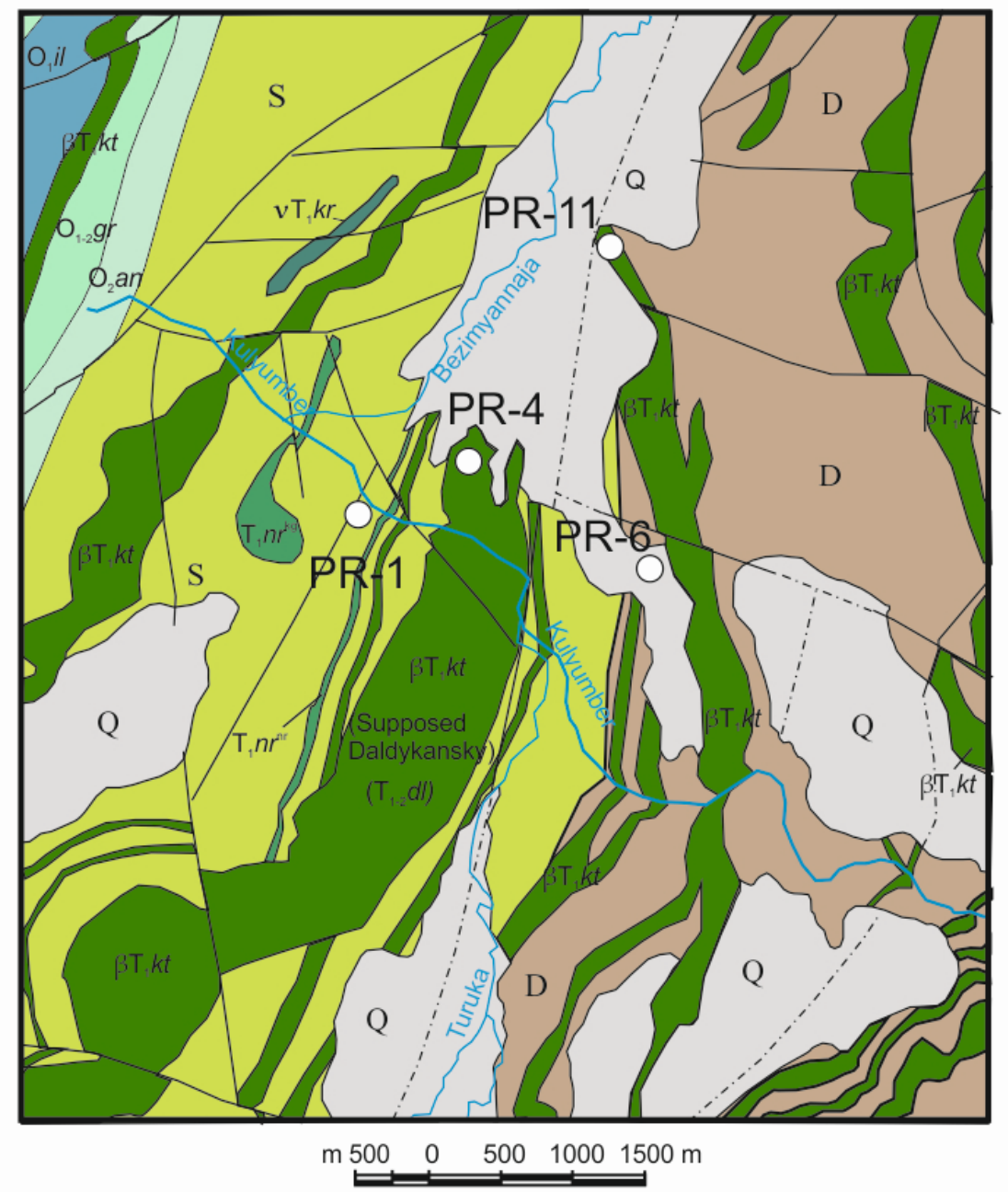

Sedimentary rocks

Q Quaternary

D Devonian

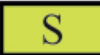

$\mathrm{O}_{2}$ an

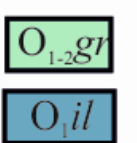

Silurian undivided

Middle Ordovican,

Angirsky formation

Lower-Middle Ordovican,

Guragirsky formation

Lower Ordovican,

Iltyksky formation
Intrusive complexes
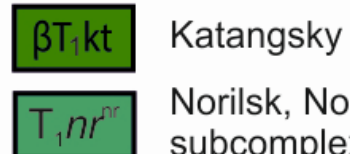

Norilsk, Norilsk

subcomplex

T.nrg Norilsk, Kruglogorsky

subcomplex

$v \mathrm{~T}_{1} \mathrm{kr}$ Kureysky

OPR-6

\section{Boreholes,} their numbers

Figure 4. Geological map and cross-section of the western Kulyumber site (after Norilskgeology Ltd. data with the authors' corrections).

Major elements in rocks were determined by X-ray fluorescence (XRF) at the Institute of Geology of Ore Deposits, Petrography, Mineralogy, and Geochemistry of Russian Academy of Science (IGEM RAS), Moscow (analyst A.I. Yakushev). Trace elements in whole rocks were analyzed by inductively coupled plasma mass spectrometry (ICP-MS) at the Institute of Microelectronics Technology and High Purity Materials RAS (IMT RAS), Chernogolovka, Russia (analyst V.K. Karandashev). These methods have been described earlier $[29,54,55]$. 
The $\mathrm{Sr}, \mathrm{Nd}$, and $\mathrm{Pb}$ isotope composition of the whole rocks was measured with the aid of a Triton (Thermo) solid-phase multi-collector mass spectrometer in static mode at the A.P. Karpinsky Russian Geological Research Institute VSEGEI Laboratory (Saint Petersburg, Russia). The values used for normalization were ${ }^{88} \mathrm{Sr} /{ }^{86} \mathrm{Sr}=8.375209$ and ${ }^{146} \mathrm{Nd}^{/ 144} \mathrm{Nd}=0.7219$. Preliminary decomposition of silicate samples and separation of elements were performed through a standard method of ion exchange column chromatography. The blank values during the analysis did not exceed $0.1 \mathrm{ng}$ for $\mathrm{Sm}, \mathrm{Nd}, \mathrm{Sr}$, and 0.05 for $\mathrm{Pb}, \mathrm{Rb}$. The isotopic compositions of the standards were JNdi-1: ${ }^{143} \mathrm{Nd} /{ }^{144} \mathrm{Nd}=0.512105 \pm$ 0.000004 and SRM987 ${ }^{87} \mathrm{Sr} /{ }^{86} \mathrm{Sr}=0.710250 \pm 0.000009$. The error of the corresponding isotope ratio was at $95 \%$ significance level in absolute values $(2 \mathrm{~s}$, abs) or percentages $(2 \mathrm{~s}, \%)$. The model age in millions of years (TMa) corresponds to the evolution model of the Earth's lead composition (Stacey-Kramers, [56]). DM1 and DM2 model ages (in million years) calculated relative to a one- and two-stage model of the mantle neodymium isotope composition evolution. The ${ }^{87} \mathrm{Sr} /{ }^{86} \mathrm{Sr}^{*}, 143 \mathrm{Nd} /{ }^{144} \mathrm{Nd}^{*}, \mathrm{eNd}(\mathrm{T})$ is the initial isotope composition of corresponding sample at the time of its origin (250 Ma).

Composition of rock-forming minerals was determined with the electron microprobe CAMECA SX 100 at the Vernadsky Institute of Geochemistry and Analytical Chemistry of Russian Academy of Science (Moscow, Russia) Analyses (analyst N.N. Kononkova) and Geol 8200 at IGEM RAS (Moscow, Russia) (analyst V. Taskaev). The device was equipped with 5 wave spectrometers. Shooting conditions: Accelerating voltage was $20 \mathrm{kV}$, a probe current was $20 \mathrm{nA}$, the diameter of the probe was $1 \mathrm{mk}$, exposure time was $10 \mathrm{sec}$. Natural minerals were used as standards (element-analytical line): Sanidine $\left(\mathrm{Si}_{\mathrm{K} \alpha}, \mathrm{Al}_{\mathrm{K} \alpha}, \mathrm{K}_{\mathrm{K} \alpha}\right)$, sc olivine $\left(\mathrm{Fe}_{\mathrm{K} \alpha}, \mathrm{Ni}_{\mathrm{K} \alpha}, \mathrm{Mg}_{\mathrm{K} \alpha}\right)$, spessartin $\left(\mathrm{Mn}_{\mathrm{K} \alpha}\right)$, anorthite $\left(\mathrm{Ca}_{\mathrm{K} \alpha}\right)$, albite $\left(\mathrm{Na}_{\mathrm{K} \alpha}\right)$, chromite $\left(\mathrm{Cr}_{\mathrm{K} \alpha}\right)$,F-phlogopite $\left(\mathrm{F}_{\mathrm{K} \alpha}\right)$, ilmenite $\left(\mathrm{Ti}_{\mathrm{K} \alpha}\right)$, and $\mathrm{Cl}$-apatite $\left(\mathrm{Cl}_{\mathrm{K} \alpha}\right)$. Element concentrations were calculated by the ZAF correction method.

\section{Results}

Here we describe the sedimentary and magmatic rocks of the third site, named the Kulyumber site (Figures 2 and 3) including its western part (Figure 4). Some intrusive bodies were studied in the cores of the boreholes drilled in the western part of this area, shown in Figure 4.

\subsection{Sedimentary Rocks}

The sedimentary rocks' outcrop is in the western part of the Kulyumber site (Figures 3 and 4), where they form the western part of the Nirungdinsky trough. The Devonian sediments in the Kulyumber river valley dip to east at 10-12 degrees. The thickness of these deposits was $50 \mathrm{~m}$. They consist of brown-red crimson, coarse-grained marls, and dark grey limestones, combined in the Nakokhozsky formation [57]. The chemical compositions of four representative samples (Kul-8-Kul-11, Table 1) had different $\mathrm{SiO}_{2} / \mathrm{CaO}$ (wt.\%) ratio varying from 0.04 to 2.01, which reflects admixture of terrigenous component in carbonate rocks. The high $\mathrm{SiO}_{2}$ and $\mathrm{MgO}$ contents are typical of marls (Table 1, Nos. 1 and 2) while low $\mathrm{SiO}_{2}$ and absence of $\mathrm{MgO}$ in rocks correspond to limestones. The contact of the Devonian deposits with overlain rocks was intruded by gabbro sills but it is described as subconformed [57]. The Tunguska Group of rocks $\left(\mathrm{C}_{2}-\mathrm{P}_{2}\right)$ comprises terrigenous rocks with coal horizons belonging to the Burguklinsky formation in this area. It is widespread in the Kulyumber river valley and consists of dark-grey, layered argillite, siltstone, and sandstone. We studied these rocks in the Khalil river valley (Table 1, Nos. 5 and 6). Quartz dominates in these rocks while carbonate and clay minerals occur as well. 
Table 1. Composition of sedimentary rocks in the Kulyumber river valley.

\begin{tabular}{|c|c|c|c|c|c|c|}
\hline No & 1 & 2 & 3 & 4 & 5 & 6 \\
\hline Sample, N & Kul-8 & Kul-9 & Kul-10 & Kul-11 & X-39-1 & $X-47-2$ \\
\hline Site & 3 & 3 & 3 & 3 & 1 & 1 \\
\hline Age & $\mathrm{D}_{3}$ & $\mathrm{D}_{3}$ & $\mathrm{D}_{3}$ & $\mathrm{D}_{3}$ & $\mathrm{C}_{2}-\mathrm{P}_{2}$ & $\mathrm{C}_{2}-\mathrm{P}_{2}$ \\
\hline Rock & marls & marls & limestone & limestone & argillite & siltstone \\
\hline $\mathrm{SiO}_{2}$ & 37.37 & 31.59 & 9.73 & 2.36 & 73.15 & 60.82 \\
\hline $\mathrm{TiO}_{2}$ & 0.57 & 0.47 & 0.2 & 0.06 & 0.42 & 0.6 \\
\hline $\mathrm{Al}_{2} \mathrm{O}_{3}$ & 8.83 & 6.69 & 2.24 & 0.6 & 9.92 & 15.42 \\
\hline $\mathrm{Fe}_{2} \mathrm{O}_{3}$ & 4.51 & 3.42 & 1.01 & 0.71 & 2.47 & 5.1 \\
\hline $\mathrm{MnO}$ & 0.09 & 0.12 & 0.05 & 0.03 & 0.09 & 0.08 \\
\hline $\mathrm{MgO}$ & 9.50 & 6.45 & 0.60 & 0.68 & 0.79 & 2.18 \\
\hline $\mathrm{CaO}$ & 18.09 & 26.03 & 48.75 & 53.84 & 4.07 & 2.98 \\
\hline $\mathrm{Na}_{2} \mathrm{O}$ & 0.08 & 0.09 & 0.19 & 0.08 & 1.11 & 5.3 \\
\hline $\mathrm{K}_{2} \mathrm{O}$ & 2.60 & 1.29 & 0.72 & 0.17 & 3.03 & 1.18 \\
\hline $\mathrm{P}_{2} \mathrm{O}_{5}$ & 0.14 & 0.11 & 0.07 & 0.03 & 0.11 & 0.23 \\
\hline $\mathrm{Cr}_{2} \mathrm{O}_{3}$ & 2.07 & 1.21 & 0.20 & 0.04 & 0.00 & 0.00 \\
\hline LOI & 18.0 & 22.6 & 35.8 & 40.9 & 4.7 & 5.5 \\
\hline$S$ & 0.07 & 1.07 & 0.51 & 0.45 & & \\
\hline Total & 101.9 & 101.1 & 100.1 & 100.0 & 99.9 & 99.4 \\
\hline $\mathrm{Li}$ & 42.8 & 49.5 & 11.2 & 7.24 & 16.6 & 16.2 \\
\hline $\mathrm{Rb}$ & 56.2 & 37.5 & 17.7 & 6.6 & 112.0 & 37.5 \\
\hline $\mathrm{Sr}$ & 257 & 134 & 103 & 236 & 63 & 177 \\
\hline $\mathrm{Y}$ & 20.1 & 14.7 & 8.00 & 1.71 & 17.0 & 23.2 \\
\hline Cs & 0.97 & 1.35 & 0.30 & 0.14 & 1.44 & 2.10 \\
\hline $\mathrm{Ba}$ & 305 & 88 & 541 & 20 & 343 & 171 \\
\hline $\mathrm{La}$ & 27.7 & 15.0 & 9.06 & 3.20 & 28.0 & 29.3 \\
\hline $\mathrm{Ce}$ & 53.7 & 30.9 & 18.3 & 5.7 & 53.8 & 60.3 \\
\hline $\operatorname{Pr}$ & 6.17 & 3.75 & 2.16 & 0.63 & 5.97 & 6.65 \\
\hline $\mathrm{Nd}$ & 22.78 & 14.36 & 8.07 & 2.24 & 20.64 & 26.40 \\
\hline $\mathrm{Sm}$ & 4.63 & 3.00 & 1.67 & 0.41 & 4.12 & 5.00 \\
\hline $\mathrm{Eu}$ & 1.08 & 0.83 & 0.44 & 0.08 & 0.85 & 1.14 \\
\hline $\mathrm{Gd}$ & 3.47 & 2.60 & 1.36 & 0.31 & 2.50 & 4.58 \\
\hline $\mathrm{Tb}$ & 0.64 & 0.45 & 0.24 & 0.05 & 0.47 & 0.70 \\
\hline Dy & 3.56 & 2.63 & 1.41 & 0.30 & 2.83 & 4.32 \\
\hline Ho & 0.77 & 0.54 & 0.29 & 0.06 & 0.60 & 0.85 \\
\hline $\mathrm{Er}$ & 2.22 & 1.56 & 0.80 & 0.18 & 1.71 & 2.57 \\
\hline $\mathrm{Tm}$ & 0.30 & 0.22 & 0.11 & 0.02 & 0.24 & 0.35 \\
\hline $\mathrm{Yb}$ & 2.02 & 1.45 & 0.70 & 0.16 & 1.48 & 2.46 \\
\hline $\mathrm{Lu}$ & 0.28 & 0.21 & 0.10 & 0.02 & 0.24 & 0.36 \\
\hline $\mathrm{Pb}$ & 2.88 & 3.91 & 4.18 & 4.39 & 4.67 & 3.13 \\
\hline $\mathrm{Th}$ & 4.93 & 3.90 & 1.11 & 0.14 & 6.21 & 8.75 \\
\hline $\mathrm{U}$ & 2.28 & 2.30 & 0.61 & 0.41 & 1.43 & 3.01 \\
\hline Sc & 8.76 & 7.32 & 3.00 & 0.95 & 31.6 & 13.2 \\
\hline $\mathrm{Ti}$ & 2778 & 2144 & 747 & 239 & 2815 & 3597 \\
\hline $\mathrm{V}$ & 75 & 56 & 19 & 7 & 58 & 100 \\
\hline $\mathrm{Cr}$ & 51.7 & 45.4 & 13.6 & 7.24 & 31.2 & 68.5 \\
\hline $\mathrm{Co}$ & 9.14 & 8.34 & 3.46 & 0.89 & 7.06 & 6.44 \\
\hline $\mathrm{Ni}$ & 25.5 & 25.9 & 7.69 & 5.04 & 11.8 & 53.6 \\
\hline $\mathrm{Cu}$ & 1.42 & 4.23 & 11.3 & 3.81 & 29.9 & 119 \\
\hline $\mathrm{Zn}$ & 30.6 & 16.4 & bdl & bdl & 11.71 & 62.1 \\
\hline $\mathrm{Zr}$ & 123 & 74.1 & 30.0 & 8.71 & 105 & 156 \\
\hline $\mathrm{Nb}$ & 15.3 & 6.44 & 2.62 & 0.30 & 14.4 & 11.3 \\
\hline Hf & 2.34 & 1.67 & 0.51 & 0.19 & 3.03 & 4.06 \\
\hline $\mathrm{Ta}$ & 0.92 & 0.40 & 0.20 & 0.02 & 1.09 & 0.73 \\
\hline
\end{tabular}

Note: Here and in Table 3, oxides are given in wt.\%, elements in ppm. Analyses number sites: 1-4 are Kulyumber and 5 and 6 are Khalil; bdl—below detected limit; LOI—loss of ignition. 
Figure 5 demonstrates the abundance of trace and rare Earth elements in the Devonian and Tunguska Group rocks. All rock samples show similar distribution patterns, though their elemental contents may differ by about two orders of magnitude. They are characterized by the Ta- $\mathrm{Nb}$-negative and $\mathrm{U}$ - and $\mathrm{Pb}$-positive anomalies; these features are typical of crust rocks. All rocks were depleted in Ti. The $(\mathrm{La} / \mathrm{Sm}) \mathrm{n}$ ratio ranged from 3.2 to 5.1 and is higher than this ratio in intrusive rocks. The $(\mathrm{Gd} / \mathrm{Yb}) \mathrm{n}$ ratio varied in narrow range from 1.4 to 1.6. The highest elements' concentrations occurred in marls and siltstones, while carbonate rocks were depleted in them. Composition of sedimentary rocks is important for the estimation of assimilation of surrounding rocks by magma during emplacement.

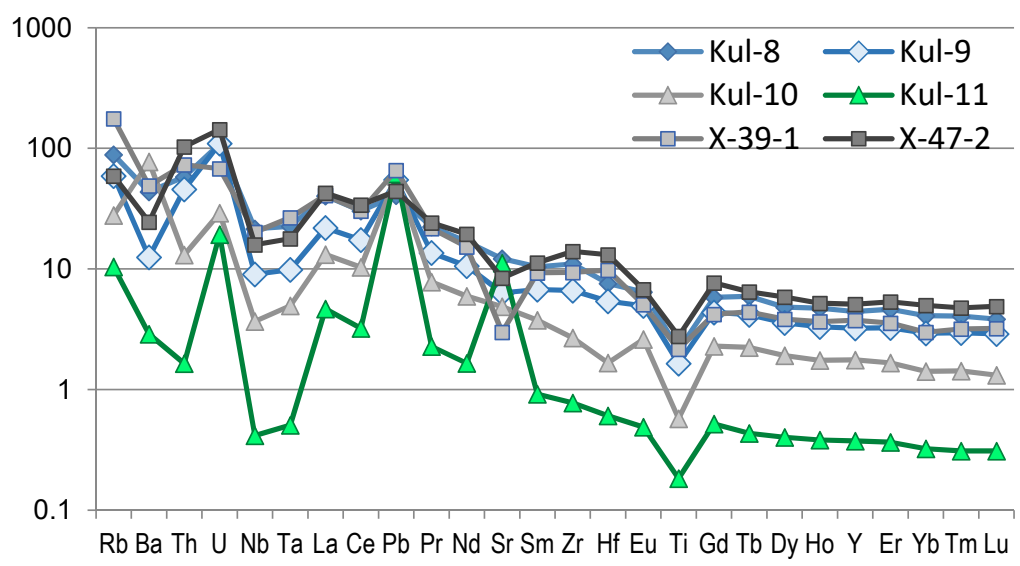

Figure 5. Trace-elements' spider diagrams for sedimentary rocks of the Kulyumber river valley, normalized to primitive mantle after [58].

\subsection{Volcanic Rocks}

Volcanic rocks form the eastern part of the area, and they occur in the center of the Nirungdinsky trough (Figure 2). They are represented by the basalts and tuffs that were correlated with volcanic rocks of the Norilsk area. Five formations were recognized within the studied area: Syverminsky, Gudchikhinsky, Khakanchansky, Tuklonsky, and Nadezhdinsky. We studied in detail the structure and composition of the lower part of the lava sequence in the Khalil site and compared it with the lower part of the volcanic section in the Norilsk area (Lake Lama). These data were published earlier [51] and volcanic rocks were not described in this article.

\subsection{Intrusive Rocks}

\subsubsection{General Characteristics of Intrusive Rocks}

As mentioned above, the study area is located at the border of two different tectonic zones (Figure 1), and this led to the formation of intrusive bodies with characteristics resembling both environments. Thus, the classification of these rocks is not straightforward. The geological map (Figures 2-4) shows that this area comprises seven different complexes including: Ergalakhsky, Norilsk, Daldykansky, Ogonersky (typical of the Norilsk area), Kuzmovsky, Kureysky, and Katangsky (typical of the Tunguska syneclise) [50,57]. The intrusive bodies constituting these complexes show similar morphology, texture, and composition. It is very difficult to recognize them without special study, on the basis of only major components in rocks. Along with the major components, we analyzed trace elements in all samples (Table 2) as well as radiogenic isotopes in some of them.

The intrusions account around 15\% of the surface (Figures 2 and 3). They are represented by sills or sill-like bodies located subconsequently in sedimentary rocks $\left(\mathrm{O}, \mathrm{S}, \mathrm{D}, \mathrm{C}_{2}-\mathrm{P}_{2}\right)$, i.e., they extend in submeridional direction at $15-20 \mathrm{~km}$ and fall to the east at $10-12^{\circ}$ to the center of the Nirungdinsky trough. Several intrusive bodies are dykes cutting volcanic rocks (site 1). The thickness of intrusions varies from 5-10 $\mathrm{m}$ to $150 \mathrm{~m}$. Initially on the surface all intrusive bodies were subdivided into 
three complexes: The Ergalakhsky of elevated alkalinity and Katangsky and Kureysky of normal alkalinity. Fine-grained gabbro-dolerites were attributed to the Katangsky complex while medium- and coarse-grained and weakly stratified rocks (up to leucogabbro) were referred to the Kureysky complex. The rocks of the first complex dominate in the Kulyumber area. The temporal relationships between intrusions of different complexes are unknown because there are no geological boundaries between them because they are separated by sedimentary rocks. Their correlation with lavas was carried out on the bases of their chemical compositions: The Ergalakhsky complex is correlated with Ivakinsky formation due to elevated Ti, alkalis, and ( $\mathrm{La} / \mathrm{Sm})$ n contents in their rocks. Katangsky and Kureysky complexes are similar to the lavas of the main stage of platform magmatism (Morongovsky-Samoedsky formations). The paleomagnetic data give similar results $[59,60]$. The most convincing results were obtained for the Ergalakhsksky complex, which is characterized by reverse magnetization like the Ivakinsky formation.

Table 2. List of studied intrusive complexes.

\begin{tabular}{|c|c|c|c|}
\hline Complex & Intrusion No, Borehole & Rocks & Sample \\
\hline Ergalakhsky & Intrusion 7 & Trachydolerites & Kul-15 \\
\hline \multirow{5}{*}{ Katangsky } & Intrusion 1 & Olivine gabbro-dolerites & $\begin{array}{c}\text { Kul-2/1, (Kul-1, } \\
\text { Kul-3-Kul-5, Kul-7, } \\
\text { Kul-38) * }\end{array}$ \\
\hline & Intrusion 2 & Olivine gabbro-dolerites & Kul-19, Kul-21, Kul-22 \\
\hline & Intrusion 6 & Gabbro-dolerites & Kul-14* \\
\hline & Intrusion 8 & Olivine gabbro-dolerites & $\begin{array}{c}\text { Kul-25, Kul-28, Kul-31, } \\
\text { Kul-32 Kul-34 }\end{array}$ \\
\hline & Intrusion 9 & Olivine gabbro-dolerites & Kul- 44 * \\
\hline \multirow{2}{*}{ Kureysky } & Intrusion 4 & $\begin{array}{c}\text { Olivine gabbro-dolerites, } \\
\text { gabbro-dolerites, Ti-Mag gabbro }\end{array}$ & $\mathrm{K}-1, \mathrm{~K}-2, \mathrm{~K}-16^{*}$ \\
\hline & Intrusion 3 & Olivine gabbro-dolerites, & Kul-40 \\
\hline Kulyumbinsky & Intrusion 5 & Gabbro-dolerites & Kul-12a, Kul-12* \\
\hline Supposed Daldykansky & PR-4, PR-11 & $\begin{array}{c}\text { Gabbro-dolerites, } \\
\text { olivine-bearing gabbro-dolerites }\end{array}$ & $\begin{array}{c}\text { PR-4/75.5, PR-4/80, } \\
\text { PR-11/486, PR-11/489.5, } \\
\text { PR-11/492.6 }\end{array}$ \\
\hline Supposed Kuzmovsky & PR-4 & Gabbro-dolerites & $\begin{array}{c}\text { PR-4/437, PR-4/440, } \\
\text { PR-4/447.5 }\end{array}$ \\
\hline Supposed Ogonersky & PR-6 & Gabbro-dolerites & PR-6/339 \\
\hline Norilsk & PR-1 & $\begin{array}{c}\text { Olivine gabbro-dolerites, } \\
\text { gabbro-dolerites, leucogabbro }\end{array}$ & $\begin{array}{c}\text { PR-1/214.6, PR-1/216, } \\
\text { PR-1/216.3, PR-1/224, } \\
\text { PR-1/234, PR-1/256, } \\
\text { PR-1/262, PR-1/262.1, } \\
\text { PR-1/266 }\end{array}$ \\
\hline
\end{tabular}

Note. *-after [60].

The rocks of the Ergalakhsky, Kureysky, Norilsk, and Katangsky complexes were recognized in outcrops while the Supposed Ogonersky, Daldykansky, Kuzmovsky, Kruglogorsky, and Norilsk subcomplex of the Norilsk complex were diagnosed in the boreholes (Figure 4, Table 2). To understand the difference between rocks of these complexes, we studied mineral and chemical compositions of rocks from the Kulyumber site and from the reference complexes (Daldykansky, Ogonersky, and Norilsk) in the Norilsk area and Katangsky and Kureysky in the Tunguska syneclise. Within the Kulyumber site (Figure 3) only one intrusive body had sub-alkaline composition and belongs to the Ergalakhsky complex (Kul-15). Three intrusive bodies were attributed to the Kureysky complex, including the large Intrusion 4 (Figure 3, samples Kul-16, K-1, K-2), one intrusion in the central part of site (sample Kul-12), and one in its right side (sample Kul-40). According to our geochemical data, these intrusive bodies belong to different complexes (see below).

Intrusions of the Katangsky complex dominate in the area. We studied five sills in the Kulyumber site across their thickness (points Kul-2-7, 13, 14) as well as along their length (Kul-25-35, 41-44). 
The intrusion penetrated by the borehole PR-1 at depths of 213-279 $\mathrm{m}$ was attributed to the Norilsk complexes (Kruglogorsky subcomplex) on the bases of the leucogabbro horizons in it and the discovery of disseminated sulfides in its lower zone (271-275 m), although it is not completely correlated with the ore-bearing intrusions of the Norilsk area. It was named the Gabbrovy intrusion. Some intrusions penetrated by boreholes PR-4 and PR-11 (Figure 4) were presumably recognized as sills of the Daldykansky (PR-4/80, PR-11/480), Ogonersky (PR-6/339), and Kuzmovsky (PR-4/430 m). We named them Supposed Daldykansky, Supposed Ogonersky, and Supposed Kuzmovsky before getting analytical data.

\subsubsection{Petrographical and Mineralogical Characteristics of Intrusive Rocks}

The Ergalakhsky complex. Trachydolerite of this complex was described in the first part of this article. It consists (vol.\%) of plagioclase $\left(\mathrm{An}_{40-55}, 50-60 \%\right)$, clinopyroxene (Wo $\mathrm{W1}_{42}$, En $\mathrm{E}_{36}-39$, and Fs $\mathrm{F}_{19-22}$, $35-40 \%$, Ti-augite), orthopyroxene (0-3\%), and olivine ( $\left.\mathrm{FO}_{50-55}, 1-3 \%\right)$.

Kureysky complex. The largest intrusion of the Kureysky intrusive complex is the Intrusion 4 in Figure 3. It is formed by coarse-grained and middle-grained olivine gabbro-dolerites and gabbro-dolerites. The main rock-forming minerals are olivine, clinopyroxene, plagioclase; moreover, rare minerals are represented by biotite, magnetite, and apatite. Composition of olivine (representative analyses of rock-forming minerals are given in Table 3, full data are in Table S1) varied significantly from $\mathrm{Fo}_{49}$ to $\mathrm{Fo}_{75}$, and the largest grains have a zoned structure where border zones are enriched in $\mathrm{Fe}$ (Figure 6a,b). The $\mathrm{CaO}$ content reached 0.32 wt.\%, and the $\mathrm{NiO}$ contents were low (0.05-0.17 wt.\%). This composition strongly distinguishes from the olivine compositions of the ore-bearing intrusions of the Norilsk area, in particular, from the Norilsk 1 intrusion (Table 3 and Table S1) and other intrusions [24,25,61-63]. Clinopyroxene has a more stable composition. Its $\mathrm{Mg}$ number ranged between 64-74. Chromium is practically absent (at the level of the electron microprobe analysis (EPMA) sensitivity) and $\mathrm{TiO}_{2}$ content reached $1 \mathrm{wt}$ \%. Maximum concentrations were (wt.\%) $\mathrm{Al}_{2} \mathrm{O}_{3}-2.25$ and $\mathrm{Na}_{2} \mathrm{O}-0.40$. The composition of plagioclase varied from $\mathrm{An}_{56}$ to $\mathrm{An}_{70}$. Magnetite contained 12-14 wt.\% $\mathrm{TiO}_{2}$. The mineral composition of the Khalil intrusion (the Khalil site), attributed to the Kureysky complex, which was also studied in the samples X-19 and was close to that of Intrusion 4 in the Kulyumber site.

Table 3. Representative compositions of rock-forming minerals, wt.\%.

\begin{tabular}{|c|c|c|c|c|c|c|c|c|c|c|c|c|}
\hline No. & $\begin{array}{c}\text { No. } \\
\text { Point }\end{array}$ & $\begin{array}{c}\text { No. } \\
\text { Sample }\end{array}$ & $\mathrm{SiO}_{2}$ & $\mathrm{TiO}_{2}$ & $\mathrm{Al}_{2} \mathrm{O}_{3}$ & $\mathrm{FeO}$ & $\mathrm{MnO}$ & $\mathrm{MgO}$ & $\mathrm{CaO}$ & $\mathrm{Na}_{2} \mathrm{O}$ & $\mathrm{NiO}$ & Total \\
\hline 1 & 1 & $\mathrm{~K}-2$ & 35.64 & 0.01 & 0.03 & 32.39 & 0.46 & 30.45 & 0.25 & bdl & 0.17 & 99.43 \\
\hline 2 & 2 & $\mathrm{~K}-2$ & 35.63 & 0.08 & 1.55 & 26.93 & 0.41 & 34.85 & 0.32 & 0.75 & 0.14 & 101.1 \\
\hline 3 & 3 & $\mathrm{~K}-2$ & 36.95 & 0.03 & 0.74 & 25.65 & 0.37 & 35.10 & 0.29 & 0.05 & 0.13 & 99.31 \\
\hline 4 & 4 & $\mathrm{~K}-2$ & 37.27 & bdl & 0.03 & 26.49 & 0.38 & 35.60 & 0.20 & 0.04 & 0.14 & 100.2 \\
\hline 5 & 11 & $\mathrm{~K}-2$ & 35.76 & bdl & bdl & 37.76 & 0.53 & 26.08 & 0.20 & bdl & 0.10 & 100.4 \\
\hline 6 & 15 & $\mathrm{~K}-2$ & 35.32 & 0.02 & 0.01 & 36.78 & 0.51 & 26.70 & 0.26 & bdl & 0.07 & 99.67 \\
\hline 7 & 26 & $\mathrm{~K}-2$ & 49.49 & 0.02 & 31.44 & 0.44 & 0.03 & 0.14 & 14.58 & 3.28 & bdl & 99.53 \\
\hline 8 & 31 & $\mathrm{~K}-2$ & 47.24 & 0.04 & 31.58 & 1.38 & bdl & 0.59 & 14.93 & 2.55 & bdl & 98.42 \\
\hline 9 & 34 & $\mathrm{~K}-2$ & 52.57 & 0.12 & 0.78 & 19.12 & 0.45 & 12.72 & 9.91 & 0.16 & bdl & 95.86 \\
\hline 10 & 35 & $\mathrm{~K}-2$ & 50.01 & 0.14 & 0.48 & 37.91 & 0.64 & 10.82 & 1.01 & 0.07 & 0.02 & 101.1 \\
\hline 11 & 38 & $\mathrm{~K}-2$ & 51.23 & 1.02 & 2.25 & 13.33 & 0.36 & 14.15 & 17.17 & 0.40 & bdl & 99.94 \\
\hline 12 & 59 & Kul-16 & 33.46 & 0.02 & 0.04 & 43.59 & 0.64 & 21.68 & 0.15 & 0.04 & 0.12 & 99.77 \\
\hline 13 & 60 & Kul-16 & 33.89 & 0.03 & 0.05 & 43.07 & 0.61 & 21.32 & 0.21 & 0.05 & 0.11 & 99.34 \\
\hline 14 & 61 & Kul-16 & 32.73 & 0.02 & 0.04 & 47.81 & 0.78 & 17.36 & 0.17 & 0.04 & 0.12 & 99.09 \\
\hline 15 & 73 & Kul-16 & 37.18 & bdl & 0.05 & 30.77 & 0.56 & 33.14 & 0.27 & 0.03 & 0.12 & 102.2 \\
\hline 16 & 74 & Kul-16 & 50.07 & 0.03 & 29.24 & 0.66 & 0.02 & 0.16 & 14.36 & 3.63 & 0.03 & 98.35 \\
\hline 17 & 75 & Kul-16 & 50.53 & 0.02 & 29.1 & 0.65 & 0.02 & 0.17 & 13.96 & 3.91 & 0.04 & 98.54 \\
\hline 18 & 103 & Kul-5 & 50.88 & bdl & 30.27 & 0.76 & bdl & 0.08 & 13.27 & 3.81 & bdl & 99.13 \\
\hline
\end{tabular}


Table 3. Cont.

\begin{tabular}{|c|c|c|c|c|c|c|c|c|c|c|c|c|}
\hline No. & $\begin{array}{c}\text { No. } \\
\text { Point }\end{array}$ & $\begin{array}{c}\text { No. } \\
\text { Sample }\end{array}$ & $\mathrm{SiO}_{2}$ & $\mathrm{TiO}_{2}$ & $\mathrm{Al}_{2} \mathrm{O}_{3}$ & $\mathrm{FeO}$ & $\mathrm{MnO}$ & $\mathrm{MgO}$ & $\mathrm{CaO}$ & $\mathrm{Na}_{2} \mathrm{O}$ & $\mathrm{NiO}$ & Total \\
\hline 19 & 104 & Kul-5 & 53.98 & bdl & 28.16 & 0.77 & bdl & 0.08 & 10.83 & 5.18 & bdl & 99.15 \\
\hline 20 & 105 & Kul-5 & 67.64 & bdl & 19.72 & 0.31 & bdl & bdl & 0.6 & 11.28 & bdl & 99.68 \\
\hline 21 & 109 & Kul-5 & 37.94 & bdl & 0.12 & 23.86 & 0.39 & 37.33 & 0.32 & bdl & 0.19 & 100.1 \\
\hline 22 & 110 & Kul-5 & 37.71 & bdl & 0.1 & 24.24 & 0.38 & 36.66 & 0.32 & bdl & 0.17 & 99.59 \\
\hline 23 & 111 & Kul-5 & 37.12 & bdl & bdl & 25.95 & 0.38 & 35.31 & 0.26 & bdl & 0.1 & 99.12 \\
\hline 24 & 112 & Kul-5 & 37.84 & bdl & bdl & 24.18 & 0.39 & 36.97 & 0.28 & bdl & 0.12 & 99.79 \\
\hline 25 & 117 & Kul-5 & 35.84 & bdl & 0.09 & 31.93 & 0.53 & 29.79 & 0.3 & bdl & bdl & 98.48 \\
\hline 26 & 118 & Kul-5 & 36.02 & bdl & 0.08 & 33.57 & 0.55 & 29.11 & 0.3 & bdl & bdl & 99.64 \\
\hline 27 & 119 & Kul-5 & 36.22 & bdl & 0.14 & 34.63 & 0.63 & 28.7 & 0.22 & bdl & bdl & 100.5 \\
\hline 28 & 120 & Kul-5 & 36.35 & bdl & 0.09 & 32.04 & 0.66 & 30.35 & 0.26 & bdl & bdl & 99.75 \\
\hline 29 & 121 & Kul-5 & 49.64 & 1.58 & 2.9 & 14.12 & 0.34 & 12.61 & 18.49 & 0.46 & bdl & 100.1 \\
\hline 30 & 122 & Kul-5 & 50.77 & 1.06 & 2.83 & 12.45 & 0.34 & 15.33 & 16.85 & .27 & bdl & 99.9 \\
\hline 31 & 123 & Kul-5 & 52.49 & 0.9 & 0.7 & 10.86 & 0.35 & 14.58 & 19.11 & 0.82 & bdl & 99.81 \\
\hline 32 & 124 & Kul-5 & 50.35 & 1.13 & 2.71 & 12.32 & 0.25 & 13.94 & 18.5 & 0.28 & bdl & 99.48 \\
\hline 33 & 133 & Kul-7 & 49.88 & 1.01 & 1.49 & 19.19 & 0.49 & 12.81 & 14.34 & .18 & bdl & 99.39 \\
\hline 34 & 135 & $\mathrm{Ku}$ & 51.05 & 0.66 & 2.76 & 9.86 & 0.25 & 15.05 & 19.77 & 33 & bdl & 99.73 \\
\hline 35 & 138 & Kul-7 & 51.07 & bdl & 30.27 & 0.76 & bdl & 0.08 & 13.27 & 81 & bdl & 99.32 \\
\hline 36 & 141 & Kul-38 & 52.7 & 0.06 & 30.28 & 0.74 & bdl & 0.11 & 14.09 & .83 & bdl & 101.9 \\
\hline 37 & 145 & Kul-38 & 50.38 & 1.22 & 3.38 & 12.57 & 0.31 & 13.68 & 18.36 & 0.33 & 0.06 & 100.6 \\
\hline 38 & 150 & $\mathrm{~K}$ & 52.31 & 0.57 & 1.17 & 8.56 & 0.25 & 17.21 & 19.37 & 0.2 & bdl & 100.0 \\
\hline 39 & 151 & Kul & 52.27 & 0.61 & 1.61 & 8.47 & 0.24 & 17.12 & 19.8 & 0.22 & bdl & 100.4 \\
\hline 40 & 152 & & 52.41 & 0.63 & 1.64 & 8.5 & 0.14 & 17.21 & 19.37 & .23 & bdl & 100.5 \\
\hline 41 & 157 & Kul & 55.78 & 0.06 & 27.3 & 0.73 & 0.01 & 0.08 & 9.31 & 6.36 & bdl & 100.0 \\
\hline 42 & 158 & Kul-12 & 55.37 & 0.02 & 27.42 & 0.72 & 0.01 & 0.11 & 9.61 & 6.32 & bdl & 100.1 \\
\hline 43 & 159 & 2 & 0.10 & 45.23 & 0.16 & 48.33 & 2.35 & 0.1 & 0.22 & 0 & $\mathrm{dl}$ & 96.83 \\
\hline 44 & 164 & Kul-14 & 38.04 & bdl & 0.1 & 24.03 & 0.38 & 38.86 & bdl & 0.05 & 0.18 & 101.6 \\
\hline 45 & 165 & & 48.18 & 0.05 & 32.62 & 0.4 & $\mathrm{bc}$ & 0.17 & 15.06 & .82 & 0.03 & 99.47 \\
\hline 46 & 166 & Kul-14 & 52.58 & 0.05 & 30.26 & 0.67 & 0.01 & 0.16 & 12.03 & 4.44 & 0.03 & 100.4 \\
\hline 47 & 187 & PR-1/234 & 36.02 & bdl & 0.08 & 33.59 & 0.55 & 30.11 & 0.3 & 0.00 & 0.01 & 99.64 \\
\hline 48 & 188 & 34 & 50.51 & 0.83 & 1.38 & 19.32 & 0.48 & 14.97 & 12.19 & 0.27 & 0.05 & 100.1 \\
\hline 49 & 189 & PR-1/ & 49.95 & 0.01 & 30.26 & 0.37 & 0.08 & 0.17 & 15.96 & 2.87 & 0.07 & 99.83 \\
\hline 50 & 191 & OKG13/43 & 36.61 & 0.03 & 0.01 & 31.46 & 0.44 & 31.75 & 0.07 & bdl & 0.01 & 100.5 \\
\hline 51 & 193 & OKG-13/43 & 36.53 & 0.03 & bdl & 31.68 & 0.44 & 31.6 & 0.08 & $\mathrm{dl}$ & bdl & 100.5 \\
\hline 52 & 194 & OKG-13/43 & 36.73 & 0.03 & 0.01 & 31.32 & 0.44 & 31.87 & 0.05 & bdl & 0.001 & 100.5 \\
\hline 53 & 195 & OKG-13/43 & 35.62 & 0.04 & bdl & 37.69 & 0.47 & 26.57 & 0.18 & bdl & 0.002 & 100.6 \\
\hline 54 & 19 & & 36.36 & & $\mathrm{~b}$ & 32.02 & 0. & 31.12 & 0.07 & $\mathrm{dl}$ & bdl & 100.1 \\
\hline 55 & 199 & ОКГ-13/69.2 & 51.63 & 0.716 & 2.11 & 8.6 & 0.226 & 15.14 & 20.73 & 0.23 & 0.066 & 99.52 \\
\hline 56 & 201 & ОКГ-13/69.2 & 51.82 & 0.469 & 1.75 & 8.23 & 0.203 & 16.37 & 19.67 & 0.17 & 0.086 & 98.79 \\
\hline 57 & 218 & 91 & 38.22 & 0.025 & 0.005 & 26.04 & 0.358 & 36.81 & 0.067 & bdl & 0.108 & 101.7 \\
\hline 58 & 219 & 91 & 37.65 & 0.020 & 0.004 & 26.09 & 0.361 & 36.82 & 0.048 & dl & 0.108 & 101.2 \\
\hline 59 & 236 & M27/20.6 & 51.47 & 0.47 & 1.35 & 954 & 0 & 14.58 & 19.8 & 0.33 & hdl & 98.04 \\
\hline 60 & 239 & M-27/20.6 & 52.14 & 0.51 & 2.62 & 6 & 0.22 & 16.11 & 20.33 & 0.25 & $\mathrm{n} / \mathrm{a}$ & 98.50 \\
\hline 61 & 241 & M-27/20.6 & 52.64 & bdl & 28.91 & 0.83 & 0.05 & bdl & 13.39 & 3.85 & $\mathrm{n} / \mathrm{a}$ & 99.67 \\
\hline 62 & 242 & M-27/20.6 & 46.77 & bdl & 28.61 & 0.58 & bdl & bdl & 12.22 & 3.43 & $\mathrm{n} / \mathrm{a}$ & 97.61 \\
\hline 63 & 243 & DM-27/20.6 & 51.67 & bdl & 30.27 & 0.67 & bdl & bdl & 13.59 & 3.63 & $\mathrm{n} / \mathrm{a}$ & 99.83 \\
\hline 64 & 245 & DM-27/32 & 51.41 & 0.74 & 1.61 & 10.29 & 0.03 & 13.91 & 20.79 & 0.43 & $n / a$ & 99.21 \\
\hline 65 & $25+2>$ & 1-27/100 & 51.79 & 0.4 & 2.29 & 6.31 & 0.16 & 16.56 & 20.94 & 0.21 & 0.2 & 98.94 \\
\hline 66 & 253 & DM-27/100 & 51.99 & 0.09 & 1.95 & 7.76 & 0.34 & 16.24 & 21.92 & 0.22 & 0.1 & 100.6 \\
\hline 67 & 256 & G-22/64 & 48.31 & 0.055 & 32.22 & 0.912 & 0.016 & 0.010 & 16.39 & 1.70 & 0.001 & 99.68 \\
\hline 68 & 264 & G-22/64 & 49.10 & 0.03 & 31.69 & 0.787 & 0.000 & 0.046 & 15.03 & 2.29 & 0.001 & 99.07 \\
\hline 69 & 271 & G-22/64 & 38.47 & $\mathrm{n} / \mathrm{a}$ & 0.020 & 19.84 & 0.310 & 40.57 & 0.16 & $\mathrm{n} / \mathrm{a}$ & 0.23 & 99.67 \\
\hline 70 & 272 & G-22/64 & 38.68 & $\mathrm{n} / \mathrm{a}$ & 0.017 & 19.38 & 0.301 & 41.00 & 0.14 & $\mathrm{n} / \mathrm{a}$ & 0.24 & 99.83 \\
\hline 71 & 273 & G-22/64 & 38.95 & $\mathrm{n} / \mathrm{a}$ & 0.016 & 19.49 & 0.303 & 41.14 & 0.15 & $n / a$ & 0.24 & 100.4 \\
\hline 72 & 289 & G-22/107 & 38.26 & $\mathrm{n} / \mathrm{a}$ & 0.021 & 24.16 & 0.394 & 37.42 & 0.149 & $\mathrm{n} / \mathrm{a}$ & 0.20 & 100.8 \\
\hline 73 & 330 & G-22/108 & 36.60 & $\mathrm{n} / \mathrm{a}$ & 0.013 & 30.44 & 0.474 & 31.71 & 0.106 & $\mathrm{n} / \mathrm{a}$ & 0.10 & 99.52 \\
\hline 74 & 331 & G-22/108 & 36.29 & $\mathrm{n} / \mathrm{a}$ & 0.020 & 29.91 & 0.466 & 31.46 & 0.112 & $n / a$ & 0.10 & 98.59 \\
\hline
\end{tabular}

Note. No. point $=$ number in Table S1 and in Figure 6. Intrusion, complex: 1-17, Intrusion 4, Kureysky complex; 18-35, Intrusion 1, Katangsky complex; 36, 37-Katangsky complex, 38-43-Kulyumbinsky complex; 44-46-Katangsky complex; 47-49, Gabbrovy intrusion; 50-58, Dzhaltulsky massif, Kureysky complex; 59-74, Norilsk 1, Norilsk complex; n/a, element was not analyzed; bdl, below detection limit. Minerals, numbers: olivine: $1-6,12-15,21-28,44-47,50-54,57,58,69-74$; pyroxene: 9-11, 29-34, 37-40, 43, 47, 55, 56, 59, 60, 64-66; plagioclase: 7, $8,16-20,35,36,41,42,45,46,49,61-63,67,68$; titanomagnetite: 43 . Analyses Nos. 37-40 contain $0.29,032,0.20$, and 0.24 wt. $\% \mathrm{Cr}_{2} \mathrm{O}_{3}$, respectively. 

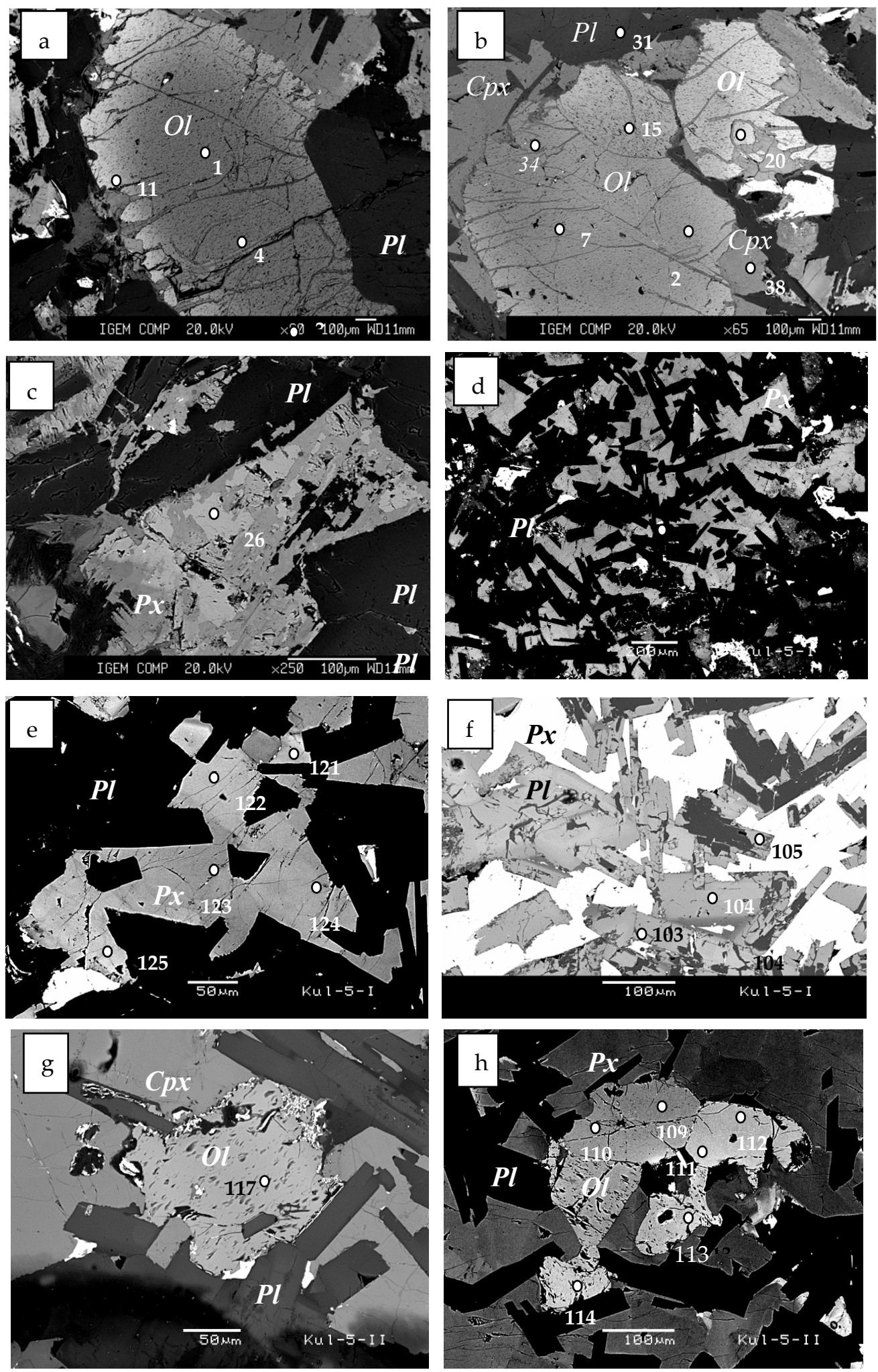

Figure 6. Cont. 

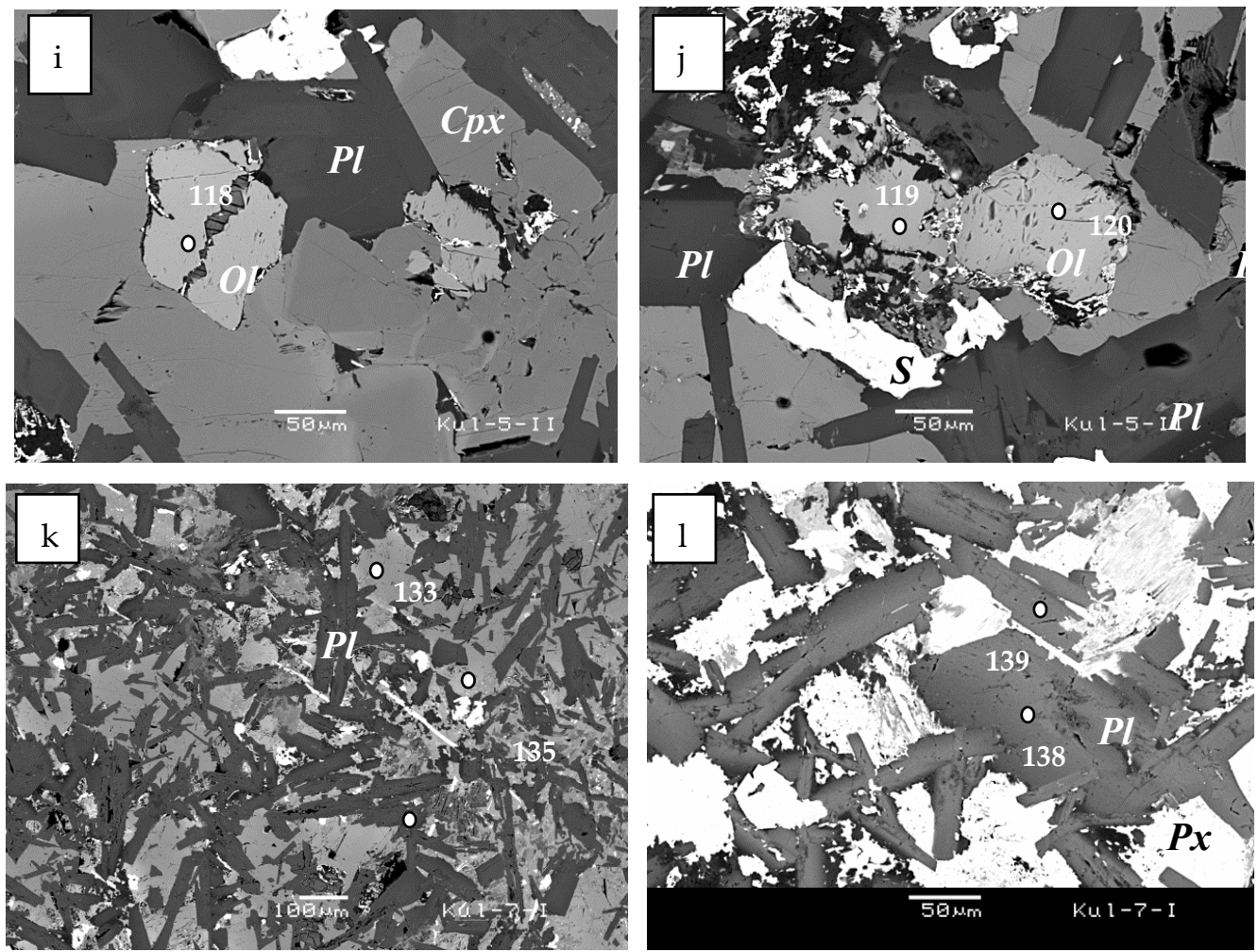

Figure 6. BSE images of the intrusive rocks $(\mathbf{a}, \mathbf{b})$ Kureysky complex, Intrusion 4, sample K-2 (c,d); (e-1) Katangsky complex; (e-j) sample Kul-5; (k-1) sample Kul-7. Minerals: Ol, olivine; Px, pyroxene; Cpx, clinipyroxene; Pl, plagioclase; S, sulfide. Point numbers correspond to numbers in Table 2.

We studied some samples from the other intrusions of this complex (Kul-40, Kul-12, Figure 4). The sample Kul-12, taken from the intrusion previously attributed to the Kureysky complex in the geological map [57] and allocated by us in a separate complex [60], consisted of plagioclase and clinopyroxene, forming the poikilophyric structure. The pyroxene has a higher Mg number (77-78) than minerals from the intrusions described before. Plagioclase was altered to sericite and $\mathrm{An}_{35}$ dominated in rocks.

The reference object of the Kureysky complex was the Dzhaltulsky massif, located several kilometers to the south from the Kulyumber area, in the Kureyka river valley. We studied three samples from the vertical section of this massif taken from the borehole OKG-13 at depth 43.7, 69.2, and $180.2 \mathrm{~m}$. One sample was taken from the surface (No. 91). Olivine changed in a wide range from Fo 55.7 to 81.6 from the upper part of the massif to its bottom. It was characterized by very low concentrations of calcium and nickel (0.07-0.17 wt.\% $\mathrm{CaO}$ and $0.03-0.06 \mathrm{wt} . \% \mathrm{NiO})$. Pyroxene composition was more stable. Its magnesium number varied from $\mathrm{Mg \#}=65$ to $\mathrm{Mg \#}=81$. It comprised a high $\mathrm{Al}_{2} \mathrm{O}_{3}$ content (up to 3 wt.\%) and sample 91 was characterized by elevated $\mathrm{Cr}_{2} \mathrm{O}_{3}$, up to $0.12 \mathrm{wt} . \%$.

Intrusions of the Katangsky complex are usually composed of fine- to medium-grained homogeneous gabbro-dolerites and olivine-bearing gabbro-dolerites. They have usually a dolerite, poikilophytic structure (Figure $6 c-f, k, l)$, where laths of plagioclase (0.1-0.3 microns) are enclosed in large grains of pyroxene (1-1.5 mm) (samples Kul-5, 7). The composition of olivine in olivine gabbro-dolerites (Kul-5, Figure 6g-j) was close to olivine composition from the Kureysky complex but differed in lower variability $\left(\mathrm{Fo}_{60-73}\right)$ and higher $\mathrm{NiO}$ contents (up to 0.19 for $\mathrm{Fo}_{73}$, it was close to olivine from the Norilsk 1 intrusion). The Mg\# number of clinopyroxene varied from 53 to 73, and orthopyroxene from 58 to 69 . The compositions of rock-forming minerals in the other samples from the Katangsky complex (samples Kul-7, Kul-14, and Kul-38) were close to the compositions for Kul-5 (Table S1). 
Supposed Norilsk complex (Kruglogorsky subcomplex). The massif, named Gabbrovy intrusion, located in the Silurian rocks (to the west from the Kulyumber site, Figure 4) was traced by several boreholes. We studied it in the core of the borehole, PR-1 penetrated at the depths from 213 to $275 \mathrm{~m}$. This intrusive body was weakly differentiated. The main volume of intrusion consisted of olivine gabbro-dolerites with leucogabbro occurring in the upper part and the thickness of $2.5 \mathrm{~m}$. This intrusion was attributed to the Norilsk complex, Kruglogorsky subcomplex, on the basis of the leucogabbro occurrence [45].

The taxitic rocks with sulfide mineralization (up to $3 \mathrm{wt} . \%, 3 \mathrm{~m}$ thick) were distinguished in the lower part of the intrusion. Sulfide minerals (pyrrhotite, chalcopyrite) reached up to $3 \mathrm{~mm}$ and they were found in the underlying sedimentary rocks as well. There were no high-magnesium rocks in this section (picrite gabbro-dolerites, troctolites) typical of the ore-bearing intrusions of the Norilsk region. The main rock-forming minerals were represented by olivine (3-20 vol. $\%$ ), plagioclase $(45-50 \%)$, clinopyroxene (15-35\%), and orthopyroxene (0-2\%). Minor minerals were titanomagnetite, ilmenite, and apatite.

Olivine was found in all varieties of rocks and its composition changed in the range of $\mathrm{Fo}_{32}-\mathrm{Fo}_{66}$. Its amount grews towads the lower part of the intrusion from the top (up to 20-25 wt.\%). Its morphology also varied. In the lower part it was represented by idiomorphic crystals, while at the top it formed interstitial grains. Clinopyroxene (augite, $\mathrm{Mg \#} \mathrm{66-68)} \mathrm{had} \mathrm{a} \mathrm{zonal} \mathrm{structure} \mathrm{where} \mathrm{the} \mathrm{central} \mathrm{parts}$ were enriched in chromium (up to $0.5 \mathrm{wt} . \% \mathrm{Cr}_{2} \mathrm{O}_{3}$ ) and border zones contained up to $1.1 \mathrm{wt} . \% \mathrm{TiO}_{2}$. Magnetite was similar in composition to magnetite from the Katangsky gabbro-dolerites (it contained up to $12 \mathrm{wt} . \% \mathrm{TiO}_{2}$ ).

\subsubsection{Chemical Composition of Intrusive Rocks}

Major components. Most of the analysis (Figure 7a, Table 4) fell in the field of basalts of normal alkalinity basalts, a number of them were located in trachybasalt and basaltic trachyandesite fields, and only several points occurred in the field of picritic basalts (Dzhaltulsky massif and Norilsk 1 intrusion). A similar distribution was observed in the $\mathrm{SiO}_{2}-\mathrm{MgO}$ diagram (Figure $7 \mathrm{~b}$ ), where only some rocks had high $\mathrm{Mg}$ compositions (23-28 wt.\% MgO) that did not belong to the Kulyumber area. Therefore, we limited further consideration of the compositions of the rocks from the Kuyumber area with $\mathrm{MgO}$ contents of 2-12 wt.\% (Figure 8). This restriction allowed us to consider the obtained data in more detail.

Table 4. Composition of intrusive rocks.

\begin{tabular}{ccccccccccc}
\hline No. & $\mathbf{1}$ & $\mathbf{2}$ & $\mathbf{3}$ & $\mathbf{4}$ & $\mathbf{5}$ & $\mathbf{6}$ & $\mathbf{7}$ & $\mathbf{8}$ & $\mathbf{9}$ & $\mathbf{1 0}$ \\
\hline Sample & $\mathbf{K u l - 1 5}$ & $\mathbf{K - 1}$ & $\mathbf{K - 2}$ & $\mathbf{K u l - 1 8}$ & $\mathbf{K u l - 4 0}$ & $\mathbf{K u l - 2 / 1}$ & $\mathbf{K u l - 2 5}$ & $\mathbf{K u l - 1 9}$ & $\mathbf{K u l - 2 2}$ & $\mathbf{K u l - 2 8}$ \\
\hline $\mathrm{SiO}_{2}$ & 50.96 & 48.06 & 47.02 & 46.62 & 48.46 & 51.88 & 47.24 & 47.58 & 47.61 & 47.59 \\
$\mathrm{TiO}_{2}$ & 2.51 & 1.34 & 1.29 & 1.52 & 1.42 & 1.43 & 1.91 & 1.46 & 1.56 & 1.31 \\
$\mathrm{Al}_{2} \mathrm{O}_{3}$ & 13.8 & 18.51 & 15.67 & 14.22 & 14.98 & 13.5 & 14.51 & 14.71 & 14.57 & 14.75 \\
$\mathrm{Fe}_{2} \mathrm{O}_{3}$ & 13.52 & 10.62 & 13.86 & 13.37 & 13.45 & 13.5 & 13.69 & 12.77 & 13.07 & 11.56 \\
$\mathrm{MnO}$ & 0.21 & 0.18 & 0.20 & 0.21 & 0.20 & 0.19 & 0.18 & 0.21 & 0.18 & 0.16 \\
$\mathrm{MgO}$ & 3.36 & 5.13 & 8.3 & 6.69 & 6.97 & 5.69 & 6.79 & 7.58 & 7.01 & 7.23 \\
$\mathrm{CaO}$ & 6.96 & 9.27 & 9.99 & 7.04 & 10.34 & 8.02 & 10.07 & 8.07 & 9.81 & 10.99 \\
$\mathrm{Na} 2 \mathrm{O}$ & 3.61 & 2.96 & 2.23 & 5.02 & 2.56 & 3.47 & 3.03 & 3.96 & 3.26 & 3.45 \\
$\mathrm{~K}_{2} \mathrm{O}$ & 2.48 & 0.85 & 0.41 & 0.53 & 0.5 & 1.38 & 0.38 & 0.78 & 0.56 & 0.52 \\
$\mathrm{P} 2 \mathrm{O} 5$ & 0.78 & 0.15 & 0.14 & 0.18 & 0.15 & 0.15 & 0.17 & 0.17 & 0.2 & 0.18 \\
$\mathrm{LOI}$ & 1.74 & 3.05 & 0.52 & 4.43 & 0.9 & 1.25 & 1.94 & 2.55 & 2.06 & 2.18 \\
$\mathrm{~S}$ & 0.04 & $n / a$ & $n / a$ & 0.05 & $n / a$ & $n / a$ & 0.08 & 0.23 & 0.09 & $\mathrm{n} / \mathrm{a}$ \\
$\mathrm{Rb}$ & 81.0 & 24.3 & 10.5 & n/a & 13.3 & 19.3 & 14.8 & 20.9 & 13.9 & 10.8 \\
$\mathrm{Sr}$ & 515 & 642 & 187 & n/a & 199 & 423 & 331 & 315 & 244 & 243 \\
\hline
\end{tabular}


Table 4. Cont

\begin{tabular}{|c|c|c|c|c|c|c|c|c|c|c|}
\hline No. & 1 & 2 & 3 & 4 & 5 & 6 & 7 & 8 & 9 & 10 \\
\hline Sample & Kul-15 & K-1 & K-2 & Kul-18 & Kul-40 & Kul-2/1 & Kul-25 & Kul-19 & Kul-22 & Kul-28 \\
\hline $\mathrm{Y}$ & 52.7 & 24.7 & 25.0 & $\mathrm{n} / \mathrm{a}$ & 25.5 & 29.5 & 35.1 & 27.8 & 29.0 & 25.7 \\
\hline Cs & 1.78 & 0.29 & 0.36 & $\mathrm{n} / \mathrm{a}$ & 0.60 & 0.29 & 0.09 & 0.20 & 0.69 & 0.07 \\
\hline $\mathrm{Ba}$ & 807 & 139 & 119 & $\mathrm{n} / \mathrm{a}$ & 112 & 172 & 148 & 122 & 144 & 110 \\
\hline $\mathrm{La}$ & 57.4 & 7.8 & 6.9 & $\mathrm{n} / \mathrm{a}$ & 7.0 & 9.4 & 10.3 & 8.5 & 9.0 & 7.5 \\
\hline $\mathrm{Ce}$ & 131 & 18.7 & 16.8 & $\mathrm{n} / \mathrm{a}$ & 16.8 & 23.5 & 25.17 & 19.7 & 21.4 & 18.4 \\
\hline $\operatorname{Pr}$ & 15.4 & 2.46 & 2.30 & $\mathrm{n} / \mathrm{a}$ & 2.41 & 3.13 & 3.64 & 2.71 & 2.91 & 2.58 \\
\hline $\mathrm{Nd}$ & 61.4 & 11.8 & 11.4 & $\mathrm{n} / \mathrm{a}$ & 11.3 & 15.0 & 17.0 & 13.3 & 14.1 & 12.3 \\
\hline Sm & 12.93 & 3.28 & 3.18 & $\mathrm{n} / \mathrm{a}$ & 3.48 & 4.00 & 4.93 & 3.83 & 4.03 & 3.57 \\
\hline $\mathrm{Eu}$ & 3.54 & 1.12 & 1.14 & $\mathrm{n} / \mathrm{a}$ & 1.19 & 1.34 & 1.62 & 1.25 & 1.31 & 1.16 \\
\hline $\mathrm{Gd}$ & 12.29 & 3.84 & 3.71 & $\mathrm{n} / \mathrm{a}$ & 3.83 & 5.04 & 5.37 & 4.80 & 5.04 & 3.86 \\
\hline $\mathrm{Tb}$ & 1.81 & 0.65 & 0.64 & $\mathrm{n} / \mathrm{a}$ & 0.69 & 0.82 & 0.99 & 0.83 & 0.85 & 0.71 \\
\hline Dy & 10.18 & 4.22 & 4.14 & $\mathrm{n} / \mathrm{a}$ & 4.69 & 5.49 & 6.41 & 5.03 & 5.26 & 4.71 \\
\hline Ho & 1.96 & 0.85 & 0.87 & $\mathrm{n} / \mathrm{a}$ & 1.00 & 1.12 & 1.35 & 1.05 & 1.06 & 1.05 \\
\hline Er & 5.37 & 2.47 & 2.47 & $\mathrm{n} / \mathrm{a}$ & 2.80 & 3.34 & 3.70 & 3.17 & 3.24 & 2.78 \\
\hline $\mathrm{Tm}$ & 0.70 & 0.35 & 0.34 & $\mathrm{n} / \mathrm{a}$ & 0.41 & 0.45 & 0.54 & 0.43 & 0.44 & 0.40 \\
\hline $\mathrm{Yb}$ & 4.77 & 2.41 & 2.50 & $\mathrm{n} / \mathrm{a}$ & 2.65 & 3.03 & 3.54 & 3.02 & 3.13 & 2.49 \\
\hline $\mathrm{Lu}$ & 0.68 & 0.34 & 0.35 & $\mathrm{n} / \mathrm{a}$ & 0.40 & 0.46 & 0.51 & 0.43 & 0.45 & 0.37 \\
\hline $\mathrm{Pb}$ & 2.41 & 0.92 & 3.62 & $\mathrm{n} / \mathrm{a}$ & 2.11 & 1.32 & 0.88 & 0.65 & 0.83 & 0.93 \\
\hline Th & 5.62 & 1.12 & 0.98 & $\mathrm{n} / \mathrm{a}$ & 0.79 & 1.47 & 1.41 & 1.22 & 1.33 & 0.98 \\
\hline $\mathrm{U}$ & 1.51 & 0.42 & 0.38 & $\mathrm{n} / \mathrm{a}$ & 0.33 & 0.59 & 0.56 & 0.45 & 0.65 & 0.39 \\
\hline Sc & 25.8 & 0.4 & 0.4 & $\mathrm{n} / \mathrm{a}$ & 36.8 & 37.8 & 50.0 & 36.7 & 37.2 & 36.6 \\
\hline $\mathrm{V}$ & 146 & $\mathrm{n} / \mathrm{a}$ & $\mathrm{n} / \mathrm{a}$ & $\mathrm{n} / \mathrm{a}$ & 279 & 319 & 329 & 288 & 306 & 244 \\
\hline $\mathrm{Cr}$ & 33 & 105 & 98 & $\mathrm{n} / \mathrm{a}$ & 168 & 165 & 249 & 151 & 153 & 181 \\
\hline $\mathrm{Co}$ & 25 & 38 & 65 & $\mathrm{n} / \mathrm{a}$ & 47 & 45 & 54 & 46 & 47 & 41 \\
\hline $\mathrm{Ni}$ & 13 & 120 & 244 & $\mathrm{n} / \mathrm{a}$ & 136 & 79 & 148 & 128 & 125 & 111 \\
\hline $\mathrm{Cu}$ & 15 & 426 & 481 & $\mathrm{n} / \mathrm{a}$ & 225 & 119 & 104 & 65 & 61 & 76 \\
\hline $\mathrm{Zn}$ & 32 & 25 & 87 & $\mathrm{n} / \mathrm{a}$ & 118 & 76 & 20 & 72 & 50 & 93 \\
\hline $\mathrm{Zr}$ & 411 & 88 & 88 & $\mathrm{n} / \mathrm{a}$ & 88 & 118 & 118 & 107 & 104 & 82 \\
\hline $\mathrm{Nb}$ & 32.61 & 4.64 & 4.29 & $\mathrm{n} / \mathrm{a}$ & 3.44 & 6.78 & 5.92 & 5.33 & 5.54 & 4.15 \\
\hline Hf & 8.95 & 2.10 & 2.30 & $\mathrm{n} / \mathrm{a}$ & 1.72 & 3.01 & 2.77 & 2.82 & 2.86 & 1.90 \\
\hline $\mathrm{Ta}$ & 1.78 & 0.26 & 0.28 & $\mathrm{n} / \mathrm{a}$ & 0.21 & 0.32 & 0.38 & 0.32 & 0.33 & 0.24 \\
\hline No. & 11 & 12 & 13 & 14 & 15 & 16 & 17 & 18 & 19 & 20 \\
\hline Sample & Kul-31 & Kul-34 & Kul-32 & Kul-24 & Kul12/1a & $1 / 214.6$ & $1 / 216.0$ & $1 / 216.3$ & $1 / 224$ & $1 / 234$ \\
\hline $\mathrm{SiO}_{2}$ & 47.06 & 47.35 & 47.60 & 47.54 & 49.37 & 47.88 & 43.05 & 46.95 & 50.04 & 47.75 \\
\hline $\mathrm{TiO}_{2}$ & 1.53 & 1.51 & 1.56 & 1.39 & 1.35 & 0.92 & 0.64 & 1.07 & 2.22 & 1.12 \\
\hline $\mathrm{Al}_{2} \mathrm{O}_{3}$ & 14.37 & 14.54 & 14.59 & 14.66 & 16.52 & 16.72 & 17.84 & 15.74 & 12.11 & 16.30 \\
\hline $\mathrm{Fe}_{2} \mathrm{O}_{3}$ & 13.61 & 13.87 & 13.86 & 12.11 & 8.53 & 7.12 & 11.24 & 11.71 & 16.10 & 12.29 \\
\hline $\mathrm{MnO}$ & 0.17 & 0.22 & 0.19 & 0.18 & 0.12 & 0.12 & 0.15 & 0.17 & 0.24 & 0.18 \\
\hline $\mathrm{MgO}$ & 7.23 & 7.31 & 7.01 & 7.26 & 7.60 & 6.80 & 10.81 & 7.46 & 4.44 & 8.30 \\
\hline $\mathrm{CaO}$ & 10.08 & 9.26 & 10.16 & 10.36 & 8.27 & 14.58 & 9.41 & 12.18 & 8.51 & 10.14 \\
\hline $\mathrm{Na}_{2} \mathrm{O}$ & 3.29 & 2.99 & 2.68 & 3.32 & 3.18 & 1.64 & 1.76 & 2.13 & 3.74 & 2.51 \\
\hline $\mathrm{K}_{2} \mathrm{O}$ & 0.44 & 0.64 & 0.47 & 0.54 & 1.73 & 2.03 & 0.79 & 0.75 & 1.13 & 0.44 \\
\hline $\mathrm{P}_{2} \mathrm{O}_{5}$ & 0.18 & 0.18 & 0.19 & 0.16 & 0.16 & 0.09 & 0.08 & 0.10 & 0.42 & 0.15 \\
\hline LOI & 1.87 & 2.05 & 1.61 & 2.34 & 2.89 & 2.05 & 4.19 & 1.73 & 0.89 & 0.61 \\
\hline$S$ & 0.18 & 0.10 & 0.04 & 0.11 & $\mathrm{n} / \mathrm{a}$ & $<0.05$ & $<0.05$ & $<0.05$ & $\mathrm{n} / \mathrm{a}$ & $\mathrm{n} / \mathrm{a}$ \\
\hline $\mathrm{Rb}$ & 11.4 & 13.4 & 12.7 & 15.1 & 53.7 & 38.9 & 18.6 & 16.7 & 28.4 & 11.5 \\
\hline $\mathrm{Sr}$ & 244 & 238 & 231 & 219 & 662 & 525 & 477 & 270 & 234 & 208 \\
\hline $\mathrm{Y}$ & 27.0 & 27.7 & 27.6 & 28 & 15.4 & 18.1 & 12.1 & 18.7 & 61.3 & 23.4 \\
\hline Cs & 0.20 & 0.20 & 0.39 & 0.6 & 4.20 & 1.67 & 3.09 & 1.55 & $\mathrm{n} / \mathrm{a}$ & $\mathrm{n} / \mathrm{a}$ \\
\hline $\mathrm{Ba}$ & 103 & 142 & 140 & 159 & 298 & 179 & 68 & 63 & 249 & 123 \\
\hline $\mathrm{La}$ & 8.4 & 8.6 & 8.93 & 8.50 & 9.03 & 4.30 & 3.30 & 4.24 & 21.39 & 7.65 \\
\hline $\mathrm{Ce}$ & 19.9 & 20.0 & 20.9 & 21.3 & 20.8 & 10.2 & 7.8 & 10.0 & 50.9 & 18.0 \\
\hline
\end{tabular}


Table 4. Cont.

\begin{tabular}{|c|c|c|c|c|c|c|c|c|c|c|}
\hline No. & 11 & 12 & 13 & 14 & 15 & 16 & 17 & 18 & 19 & 20 \\
\hline Sample & Kul-31 & Kul-34 & Kul-32 & Kul-24 & Kul12/1a & $1 / 214.6$ & $1 / 216.0$ & $1 / 216.3$ & $1 / 224$ & $1 / 234$ \\
\hline $\operatorname{Pr}$ & 2.70 & 2.71 & 2.93 & 2.90 & 2.66 & 1.45 & 1.08 & 1.39 & 6.79 & 2.45 \\
\hline $\mathrm{Nd}$ & 13.1 & 13.4 & 13.54 & 13.1 & 12.3 & 7.5 & 5.3 & 7.1 & 31.1 & 11.1 \\
\hline $\mathrm{Sm}$ & 3.77 & 3.88 & 3.82 & 3.94 & 3.02 & 2.19 & 1.43 & 2.13 & 9.08 & 3.51 \\
\hline $\mathrm{Eu}$ & 1.14 & 1.29 & 1.27 & 1.30 & 1.09 & 0.79 & 0.58 & 0.78 & 2.35 & 1.06 \\
\hline $\mathrm{Gd}$ & 4.74 & 4.86 & 4.20 & 4.17 & 3.36 & 2.96 & 1.93 & 2.86 & 9.85 & 3.59 \\
\hline $\mathrm{Tb}$ & 0.79 & 0.82 & 0.77 & 0.78 & 0.52 & 0.48 & 0.32 & 0.48 & 1.68 & 0.63 \\
\hline Dy & 5.02 & 5.01 & 5.07 & 5.03 & 3.18 & 3.24 & 2.14 & 3.31 & 10.9 & 4.15 \\
\hline Ho & 1.03 & 1.04 & 1.08 & 1.10 & 0.59 & 0.65 & 0.43 & 0.68 & 2.26 & 0.89 \\
\hline Er & 3.10 & 3.14 & 2.98 & 3.00 & 1.56 & 1.88 & 1.27 & 2.00 & 6.43 & 2.44 \\
\hline $\mathrm{Tm}$ & 0.41 & 0.43 & 0.44 & 0.43 & 0.22 & 0.27 & 0.19 & 0.29 & 0.94 & 0.36 \\
\hline $\mathrm{Yb}$ & 2.94 & 2.94 & 2.85 & 2.76 & 1.48 & 1.80 & 1.26 & 1.86 & 6.07 & 2.40 \\
\hline $\mathrm{Lu}$ & 0.42 & 0.43 & 0.42 & 0.43 & 0.22 & 0.27 & 0.18 & 0.28 & 0.92 & 0.35 \\
\hline $\mathrm{Pb}$ & 0.74 & 1.10 & 1.73 & 3.82 & 0.54 & 1.17 & 0.71 & 0.74 & 5.17 & 2.33 \\
\hline Th & 1.20 & 1.17 & 1.08 & 1.10 & 0.98 & 0.63 & 0.54 & 0.63 & 3.21 & 1.08 \\
\hline $\mathrm{U}$ & 0.55 & 0.50 & 0.57 & 0.43 & 0.23 & 0.26 & 0.22 & 0.26 & 1.37 & 0.44 \\
\hline Sc & 38.0 & 38.7 & 37.1 & 37.0 & 25.4 & 52.9 & 17.2 & 48.0 & 39.8 & 27.9 \\
\hline $\mathrm{V}$ & 313 & 315 & 295 & 301 & 220 & 311 & 139 & 304 & 327 & 223 \\
\hline $\mathrm{Cr}$ & 156 & 161 & 157 & 126 & 317 & 253 & 82 & 408 & 36 & 131 \\
\hline $\mathrm{Co}$ & 50 & 52 & 48.8 & 47.8 & 21.6 & 29.8 & 64.5 & 42.4 & 38.5 & 53.4 \\
\hline $\mathrm{Ni}$ & 125 & 127 & 123 & 128 & 118 & 88 & 393 & 119 & 57 & 170 \\
\hline $\mathrm{Cu}$ & 165 & 153 & 179 & 248 & 81 & 78 & 31 & 68 & 284 & 131 \\
\hline $\mathrm{Zn}$ & 62 & 84 & 133 & 194 & 28 & 41 & 59 & 49 & 110 & $<\Pi \mathrm{O}$ \\
\hline $\mathrm{Zr}$ & 99 & 101 & 71 & 110 & 115 & 55 & 40 & 60 & 208 & 74 \\
\hline $\mathrm{Nb}$ & 5.10 & 5.10 & 4.63 & 4.00 & 6.92 & 2.68 & 2.80 & 2.71 & 10.74 & 3.86 \\
\hline Hf & 2.68 & 2.75 & 2.26 & 2.3 & 2.17 & 1.53 & 1.08 & 1.49 & 5.16 & 1.85 \\
\hline $\mathrm{Ta}$ & 0.30 & 0.32 & 0.29 & 0.21 & 0.38 & 0.19 & 0.19 & 0.18 & 0.67 & 0.27 \\
\hline No. & 21 & 22 & 23 & 24 & 25 & 26 & 27 & 28 & 29 & 30 \\
\hline Sample & $1 / 256$ & $1 / 262$ & 1/262.1 & $1 / 266$ & $4 / 75.5$ & $4 / 80$ & $11 / 486$ & $11 / 489.5$ & $11 / 492.6$ & $6-339$ \\
\hline $\mathrm{SiO}_{2}$ & 48.07 & 46.36 & 47.14 & 47.61 & 46.53 & 46.72 & 47.86 & 48.45 & 46.55 & 47.64 \\
\hline $\mathrm{TiO}_{2}$ & 1.22 & 1.32 & 1.31 & 1.29 & 1.65 & 1.57 & 1.38 & 2.02 & 1.33 & 1.31 \\
\hline $\mathrm{Al}_{2} \mathrm{O}_{3}$ & 16.19 & 14.81 & 15.08 & 16.41 & 15.03 & 14.78 & 15.13 & 13.56 & 15.62 & 14.98 \\
\hline $\mathrm{Fe}_{2} \mathrm{O}_{3}$ & 12.42 & 13.52 & 13.31 & 12.06 & 13.81 & 13.95 & 12.51 & 13.55 & 12.61 & 13.25 \\
\hline $\mathrm{MnO}$ & 0.18 & 0.19 & 0.19 & 0.17 & 0.19 & 0.20 & 0.18 & 0.22 & 0.18 & 0.21 \\
\hline $\mathrm{MgO}$ & 8.21 & 9.21 & 9.05 & 7.90 & 7.09 & 7.15 & 7.37 & 5.54 & 7.76 & 7.63 \\
\hline $\mathrm{CaO}$ & 10.28 & 10.26 & 9.83 & 10.54 & 10.32 & 10.13 & 11.16 & 11.28 & 10.05 & 11.34 \\
\hline $\mathrm{Na}_{2} \mathrm{O}$ & 2.43 & 2.44 & 2.45 & 2.46 & 2.54 & 2.51 & 2.50 & 2.82 & 2.38 & 2.42 \\
\hline $\mathrm{K}_{2} \mathrm{O}$ & 0.44 & 0.48 & 0.49 & 0.48 & 0.47 & 0.43 & 0.42 & 0.76 & 0.44 & 0.48 \\
\hline $\mathrm{P}_{2} \mathrm{O}_{5}$ & 0.16 & 0.17 & 0.17 & 0.15 & 0.16 & 0.15 & 0.14 & 0.21 & 0.15 & 0.15 \\
\hline LOI & 0.29 & 1.1 & 0.84 & 0.77 & 2.14 & 2.36 & 1.27 & 1.23 & 2.87 & 0.37 \\
\hline$S$ & $\mathrm{n} / \mathrm{a}$ & $\mathrm{n} / \mathrm{a}$ & $\mathrm{n} / \mathrm{a}$ & $\mathrm{n} / \mathrm{a}$ & $<0.05$ & $<0.05$ & $<0.05$ & 0.34 & $<0.05$ & $\mathrm{n} / \mathrm{a}$ \\
\hline $\mathrm{Rb}$ & 11.8 & $\mathrm{n} / \mathrm{a}$ & 13.8 & 12.7 & 11.1 & 11.6 & 9.8 & 20.0 & 11.6 & 12.1 \\
\hline Sr & 204 & $\mathrm{n} / \mathrm{a}$ & 208 & 224 & 196 & 199 & 196 & 427 & 204 & 255 \\
\hline $\mathrm{Y}$ & 24.4 & $\mathrm{n} / \mathrm{a}$ & 25.4 & 25.0 & 28.1 & 29.7 & 23.3 & 35.8 & 23.5 & 24.0 \\
\hline Cs & $\mathrm{n} / \mathrm{a}$ & $\mathrm{n} / \mathrm{a}$ & $\mathrm{n} / \mathrm{a}$ & $\mathrm{n} / \mathrm{a}$ & 0.31 & 0.34 & 0.39 & 1.40 & 0.39 & 0.41 \\
\hline $\mathrm{Ba}$ & 125 & $\mathrm{n} / \mathrm{a}$ & 132 & 132 & 112 & 117 & 116 & 169 & 124 & 114 \\
\hline $\mathrm{La}$ & 7.66 & $\mathrm{n} / \mathrm{a}$ & 8.26 & 8.13 & 7.23 & 7.68 & 6.17 & 10.74 & 6.69 & 6.83 \\
\hline $\mathrm{Ce}$ & 18.3 & $\mathrm{n} / \mathrm{a}$ & 19.6 & 19.4 & 17.7 & 18.6 & 14.8 & 25.5 & 15.8 & 16.0 \\
\hline Pr & 2.48 & $\mathrm{n} / \mathrm{a}$ & 2.70 & 2.63 & 2.47 & 2.60 & 2.03 & 3.42 & 2.15 & 2.18 \\
\hline $\mathrm{Nd}$ & 11.7 & $\mathrm{n} / \mathrm{a}$ & 12.4 & 11.9 & 12.3 & 13.0 & 10.1 & 16.5 & 10.7 & 10.5 \\
\hline Sm & 3.60 & $\mathrm{n} / \mathrm{a}$ & 3.85 & 3.68 & 3.53 & 3.75 & 2.89 & 4.57 & 2.99 & 2.98 \\
\hline $\mathrm{Eu}$ & 1.12 & $\mathrm{n} / \mathrm{a}$ & 1.13 & 1.12 & 1.25 & 1.38 & 1.08 & 1.52 & 1.10 & 1.08 \\
\hline $\mathrm{Gd}$ & 3.79 & $\mathrm{n} / \mathrm{a}$ & 4.05 & 3.87 & 4.56 & 4.80 & 3.69 & 5.75 & 3.66 & 3.81 \\
\hline $\mathrm{Tb}$ & 0.68 & $\mathrm{n} / \mathrm{a}$ & 0.71 & 0.68 & 0.75 & 0.80 & 0.61 & 0.93 & 0.62 & 0.63 \\
\hline
\end{tabular}


Table 4. Cont.

\begin{tabular}{|c|c|c|c|c|c|c|c|c|c|c|c|}
\hline No. & 21 & 22 & 23 & 24 & 25 & & 26 & 27 & 28 & 29 & 30 \\
\hline Sample & $1 / 256$ & $1 / 262$ & $1 / 262.1$ & $1 / 266$ & $4 / 75.5$ & & $/ 80$ & $11 / 486$ & $11 / 489.5$ & $11 / 492.6$ & $6-339$ \\
\hline Dy & 4.41 & $\mathrm{n} / \mathrm{a}$ & 4.58 & 4.47 & 4.99 & & .27 & 4.09 & 6.28 & 4.11 & 4.28 \\
\hline Ho & 0.92 & $\mathrm{n} / \mathrm{a}$ & 0.95 & 0.94 & 1.00 & & .08 & 0.81 & 1.26 & 0.81 & 0.84 \\
\hline Er & 2.61 & $\mathrm{n} / \mathrm{a}$ & 2.60 & 2.70 & 2.93 & & 15 & 2.41 & 3.76 & 2.43 & 2.51 \\
\hline $\mathrm{Tm}$ & 0.38 & $\mathrm{n} / \mathrm{a}$ & 0.40 & 0.41 & 0.42 & & .45 & 0.34 & 0.53 & 0.34 & 0.35 \\
\hline $\mathrm{Yb}$ & 2.47 & $\mathrm{n} / \mathrm{a}$ & 2.51 & 2.51 & 2.77 & & .93 & 2.31 & 3.57 & 2.29 & 2.36 \\
\hline $\mathrm{Lu}$ & 0.37 & $\mathrm{n} / \mathrm{a}$ & 0.37 & 0.38 & 0.41 & & .44 & 0.34 & 0.52 & 0.34 & 0.35 \\
\hline $\mathrm{Pb}$ & 2.26 & $\mathrm{n} / \mathrm{a}$ & 3.36 & 2.71 & 2.00 & & .09 & 1.94 & 2.31 & 2.00 & 3.62 \\
\hline Th & 1.10 & $\mathrm{n} / \mathrm{a}$ & 1.13 & 1.29 & 1.06 & & .09 & 0.89 & 1.66 & 0.94 & 1.02 \\
\hline $\mathrm{U}$ & 0.47 & $\mathrm{n} / \mathrm{a}$ & 0.45 & 0.70 & 0.36 & & .38 & 0.34 & 0.61 & 0.36 & 0.44 \\
\hline Sc & 30.1 & $\mathrm{n} / \mathrm{a}$ & 27.6 & 28.3 & 31.7 & & 6.5 & 36.8 & 38.1 & 29.6 & 33.5 \\
\hline $\mathrm{V}$ & 241 & $\mathrm{n} / \mathrm{a}$ & 239 & 255 & 287 & & 311 & 317 & 350 & 266 & 280 \\
\hline $\mathrm{Cr}$ & 149 & $\mathrm{n} / \mathrm{a}$ & 145 & 108 & 150 & & 91 & 300 & 86 & 191 & 245 \\
\hline Co & 51.6 & $\mathrm{n} / \mathrm{a}$ & 57.2 & 49.2 & 52.0 & & 7.3 & 46.5 & 37.4 & 52 & 49 \\
\hline $\mathrm{Ni}$ & 184 & $\mathrm{n} / \mathrm{a}$ & 259 & 206 & 136 & & 28 & 124 & 62 & 178 & 142 \\
\hline $\mathrm{Cu}$ & 157 & $\mathrm{n} / \mathrm{a}$ & 170 & 154 & 189 & & 97 & 147 & 241 & 158 & 138 \\
\hline $\mathrm{Zn}$ & $<\Pi \mathrm{O}$ & $\mathrm{n} / \mathrm{a}$ & 62 & $<\Pi \mathrm{O}$ & 105 & & 08 & 94 & 75 & 99 & 91 \\
\hline $\mathrm{Zr}$ & 80 & $\mathrm{n} / \mathrm{a}$ & 82 & 82 & 108 & & 09 & 84 & 135 & 87 & 90 \\
\hline $\mathrm{Nb}$ & 4.42 & $\mathrm{n} / \mathrm{a}$ & 4.37 & 4.46 & 2.70 & & .71 & 1.98 & 3.36 & 2.08 & 2.19 \\
\hline Hf & 2.04 & $\mathrm{n} / \mathrm{a}$ & 1.83 & 2.19 & 0.34 & & .34 & 0.25 & 0.41 & 0.28 & 0.27 \\
\hline $\mathrm{Ta}$ & 0.28 & $\mathrm{n} / \mathrm{a}$ & 0.29 & 0.24 & 0.20 & & .20 & 0.19 & 0.17 & 0.14 & 0.17 \\
\hline $\begin{array}{l}\text { No. } \\
\text { Sample }\end{array}$ & $\begin{array}{c}31 \\
4 / 437\end{array}$ & $\begin{array}{c}32 \\
4 / 440\end{array}$ & $\begin{array}{c}33 \\
4 / 443.5\end{array}$ & $\begin{array}{c}34 \\
\text { DL-2 }\end{array}$ & $\begin{array}{c}35 \\
\text { DL-1 }\end{array}$ & $\begin{array}{l}36 \\
\text { DL-3 }\end{array}$ & $\begin{array}{c}37 \\
\text { Val-2 }\end{array}$ & $\begin{array}{c}38 \\
\text { Val-1 }\end{array}$ & $\begin{array}{c}39 \\
\text { Og-1 }\end{array}$ & $\begin{array}{c}40 \\
\text { Og-2 }\end{array}$ & $\begin{array}{c}41 \\
\text { Og-3 }\end{array}$ \\
\hline $\mathrm{SiO}_{2}$ & 47.32 & 47.87 & 48.23 & 49.47 & 49.60 & 49.20 & 50.41 & 50.03 & 49.23 & 48.93 & 48.58 \\
\hline $\mathrm{TiO}_{2}$ & 1.49 & 1.42 & 1.24 & 1.71 & 1.61 & 1.73 & 1.64 & 1.92 & 1.32 & 1.43 & 1.16 \\
\hline $\mathrm{Al}_{2} \mathrm{O}_{3}$ & 14.40 & 15.83 & 15.41 & 15.77 & 15.55 & 15.06 & 14.74 & 14.30 & 15.74 & 15.81 & 17.15 \\
\hline $\mathrm{Fe}_{2} \mathrm{O}_{3}$ & 13.22 & 12.37 & 12.54 & 12.89 & 12.79 & 13.66 & 13.22 & 13.49 & 12.16 & 12.89 & 11.78 \\
\hline $\mathrm{MnO}$ & 0.20 & 0.18 & 0.18 & 0.26 & 0.22 & 0.27 & 0.25 & 0.24 & 0.22 & 0.21 & 0.19 \\
\hline $\mathrm{MgO}$ & 6.98 & 6.39 & 7.23 & 5.93 & 6.20 & 5.94 & 6.09 & 5.79 & 6.54 & 6.70 & 7.77 \\
\hline $\mathrm{CaO}$ & 10.79 & 10.64 & 11.01 & 10.29 & 10.15 & 10.30 & 10.30 & 10.80 & 11.23 & 10.53 & 10.07 \\
\hline $\mathrm{Na}_{2} \mathrm{O}$ & 2.55 & 2.70 & 2.56 & 2.96 & 2.91 & 2.92 & 2.71 & 2.66 & 2.59 & 2.62 & 2.70 \\
\hline $\mathrm{K}_{2} \mathrm{O}$ & 0.48 & 0.55 & 0.44 & 0.57 & 0.59 & 0.54 & 0.65 & 0.63 & 0.46 & 0.47 & 0.40 \\
\hline $\mathrm{P}_{2} \mathrm{O}_{5}$ & 0.16 & 0.16 & 0.14 & 0.17 & 0.19 & 0.23 & 0.21 & 0.15 & 0.16 & 0.17 & 0.10 \\
\hline LOI & 2.36 & 1.83 & 0.95 & $\mathrm{n} / \mathrm{a}$ & $\mathrm{n} / \mathrm{a}$ & $\mathrm{n} / \mathrm{a}$ & $\mathrm{n} / \mathrm{a}$ & $\mathrm{n} / \mathrm{a}$ & $\mathrm{n} / \mathrm{a}$ & $\mathrm{n} / \mathrm{a}$ & $\mathrm{n} / \mathrm{a}$ \\
\hline$S$ & $<0.05$ & $<0.05$ & $<0.05$ & $\mathrm{n} / \mathrm{a}$ & $\mathrm{n} / \mathrm{a}$ & $\mathrm{n} / \mathrm{a}$ & $\mathrm{n} / \mathrm{a}$ & $\mathrm{n} / \mathrm{a}$ & $\mathrm{n} / \mathrm{a}$ & $\mathrm{n} / \mathrm{a}$ & $\mathrm{n} / \mathrm{a}$ \\
\hline $\mathrm{Rb}$ & 10.7 & 13.4 & 10.8 & 14.5 & 15.4 & 13.2 & 18.6 & 18.5 & 13.2 & 13.8 & 11.0 \\
\hline $\mathrm{Sr}$ & 175 & 194 & 192 & 223 & 227 & 204 & 230 & 216 & 240 & 238 & 225 \\
\hline $\mathrm{Y}$ & 23.8 & 27.3 & 23.9 & 31.5 & 32.1 & 33.4 & 31.6 & 32.0 & 29.6 & 26.4 & 18.2 \\
\hline Cs & 0.39 & 0.37 & 0.42 & $\mathrm{n} / \mathrm{a}$ & $\mathrm{n} / \mathrm{a}$ & $\mathrm{n} / \mathrm{a}$ & $\mathrm{n} / \mathrm{a}$ & $\mathrm{n} / \mathrm{a}$ & $\mathrm{n} / \mathrm{a}$ & $\mathrm{n} / \mathrm{a}$ & $\mathrm{n} / \mathrm{a}$ \\
\hline $\mathrm{Ba}$ & 120 & 140 & 118 & 168 & 146 & 153 & 161 & 144 & 123 & 144 & 117 \\
\hline $\mathrm{La}$ & 6.72 & 8.04 & 6.68 & 10.9 & 10.1 & 10.8 & 10.7 & 10.2 & 8.89 & 8.43 & 5.79 \\
\hline $\mathrm{Ce}$ & 16.1 & 19.2 & 15.8 & 23.15 & 22.0 & 24.0 & 22.1 & 22.9 & 20.4 & 19.7 & 13.4 \\
\hline $\operatorname{Pr}$ & 2.26 & 2.61 & 2.18 & 3.19 & 3.14 & 3.34 & 3.09 & 3.18 & 2.77 & 2.63 & 1.76 \\
\hline $\mathrm{Nd}$ & 10.8 & 12.7 & 10.7 & 15.9 & 15.7 & 16.4 & 15.5 & 15.6 & 13.5 & 13.1 & 8.47 \\
\hline $\mathrm{Sm}$ & 3.14 & 3.55 & 3.08 & 4.4 & 4.49 & 4.70 & 4.38 & 4.58 & 3.89 & 3.55 & 2.40 \\
\hline $\mathrm{Eu}$ & 1.16 & 1.24 & 1.14 & 1.62 & 1.66 & 1.67 & 1.56 & 1.58 & 1.50 & 1.42 & 1.13 \\
\hline $\mathrm{Gd}$ & 4.03 & 4.44 & 3.86 & 4.91 & 4.96 & 5.22 & 4.78 & 4.90 & 4.52 & 4.06 & 2.83 \\
\hline $\mathrm{Tb}$ & 0.66 & 0.71 & 0.63 & 0.86 & 0.89 & 0.93 & 0.85 & 0.89 & 0.78 & 0.72 & 0.49 \\
\hline Dy & 4.29 & 4.84 & 4.29 & 6.17 & 6.39 & 6.17 & 6.25 & 6.02 & 5.58 & 4.92 & 3.31 \\
\hline Ho & 0.87 & 0.94 & 0.85 & 1.28 & 1.29 & 1.38 & 1.25 & 1.27 & 1.18 & 1.02 & 0.70 \\
\hline $\mathrm{Er}$ & 2.61 & 2.78 & 2.53 & 3.22 & 3.23 & 3.46 & 3.14 & 3.20 & 3.02 & 2.71 & 1.86 \\
\hline $\mathrm{Tm}$ & 0.35 & 0.39 & 0.36 & 0.42 & 0.44 & 0.50 & 0.46 & 0.45 & 0.42 & 0.39 & 0.26 \\
\hline $\mathrm{Yb}$ & 2.40 & 2.63 & 2.38 & 3.09 & 3.23 & 3.48 & 3.33 & 3.34 & 2.99 & 2.76 & 1.87 \\
\hline
\end{tabular}


Table 4. Cont.

\begin{tabular}{|c|c|c|c|c|c|c|c|c|c|c|c|}
\hline $\begin{array}{l}\text { No. } \\
\text { Sample }\end{array}$ & $\begin{array}{c}31 \\
4 / 437\end{array}$ & $\begin{array}{c}32 \\
4 / 440\end{array}$ & $\begin{array}{c}33 \\
4 / 443.5\end{array}$ & $\begin{array}{c}34 \\
\text { DL-2 }\end{array}$ & $\begin{array}{c}35 \\
\text { DL-1 }\end{array}$ & $\begin{array}{l}36 \\
\text { DL-3 }\end{array}$ & $\begin{array}{c}37 \\
\text { Val-2 }\end{array}$ & $\begin{array}{c}38 \\
\text { Val-1 }\end{array}$ & $\begin{array}{c}39 \\
\text { Og-1 }\end{array}$ & $\begin{array}{c}40 \\
\text { Og-2 }\end{array}$ & $\begin{array}{c}41 \\
\text { Og-3 }\end{array}$ \\
\hline $\mathrm{Lu}$ & 0.36 & 0.39 & 0.35 & 0.48 & 0.44 & 0.50 & 0.47 & 0.47 & 0.44 & 0.39 & 0.27 \\
\hline $\mathrm{Pb}$ & 2.22 & 2.64 & 1.86 & 0.73 & 1.51 & 3.11 & 3.26 & 1.49 & 0.73 & 0.97 & 0.56 \\
\hline Th & 0.88 & 1.14 & 0.93 & 1.27 & 0.96 & 1.49 & 1.46 & 1.38 & 1.17 & 1.10 & 0.73 \\
\hline $\mathrm{U}$ & 0.32 & 0.43 & 0.36 & 0.45 & 0.34 & 0.52 & 0.45 & 0.49 & 0.38 & 0.36 & 0.23 \\
\hline Sc & 38.6 & 32.5 & 35.0 & 34.1 & 37.3 & 38.7 & 43.0 & 44.1 & 40.0 & 34.4 & 27.3 \\
\hline V & 312 & 261 & 271 & 273 & 304 & 342 & 327 & 364 & 298 & 303 & 244 \\
\hline $\mathrm{Cr}$ & 204 & 145 & 242 & $\mathrm{n} / \mathrm{a}$ & $\mathrm{n} / \mathrm{a}$ & $\mathrm{n} / \mathrm{a}$ & $\mathrm{n} / \mathrm{a}$ & $\mathrm{n} / \mathrm{a}$ & $\mathrm{n} / \mathrm{a}$ & $\mathrm{n} / \mathrm{a}$ & $\mathrm{n} / \mathrm{a}$ \\
\hline Co & 49 & 46 & 48 & 46 & 48 & 47 & 49 & 49 & 50 & 54 & 59 \\
\hline $\mathrm{Ni}$ & 119 & 105 & 122 & 100 & 111 & 100 & 80 & 66 & 115 & 126 & 174 \\
\hline $\mathrm{Cu}$ & 141 & 150 & 154 & 226 & 210 & 308 & 212 & 222 & 217 & 182 & 146 \\
\hline $\mathrm{Zn}$ & 96 & 94 & 101 & 71 & 100 & 154 & 129 & 92 & 76 & 84 & 49 \\
\hline $\mathrm{Zr}$ & 80 & 85 & 77 & 133 & 101 & 121 & 123 & 126 & 91 & 95 & 65 \\
\hline $\mathrm{Nb}$ & 1.97 & 2.19 & 1.96 & 6.84 & 6.55 & 7.37 & 6.31 & 6.84 & 4.79 & 4.81 & 4.36 \\
\hline Hf & 0.29 & 0.32 & 0.27 & 3.47 & 2.39 & 3.52 & 3.01 & 2.99 & 2.34 & 2.47 & 1.70 \\
\hline $\mathrm{Ta}$ & 0.13 & 0.13 & 0.13 & 0.44 & 0.41 & 0.45 & 0.49 & 0.39 & 0.28 & 0.31 & 0.27 \\
\hline
\end{tabular}

Note: Oxides are given in wt.\%, elements are in ppm. Nos. (N), borehole/depth (m): 16-24 PR, 25-26 PR-4, 27-29 PR-11, 30 PR-6, 31-33 PR-4; n/a, element was not analyzed; Intrusive complexes, No: Katangsky-1, 6-14; Kureysky, 2-5; Kulyumbinsky, 15; Norilsk, Kruglogorsky subcomplex, 16-24; Supposed Daldykansky, 25-29; Supposed Ogonersky, 30; Supposed Kuzmovsky, 31-33; Daldykansky, 34-38; Ogonersky, 39-41.

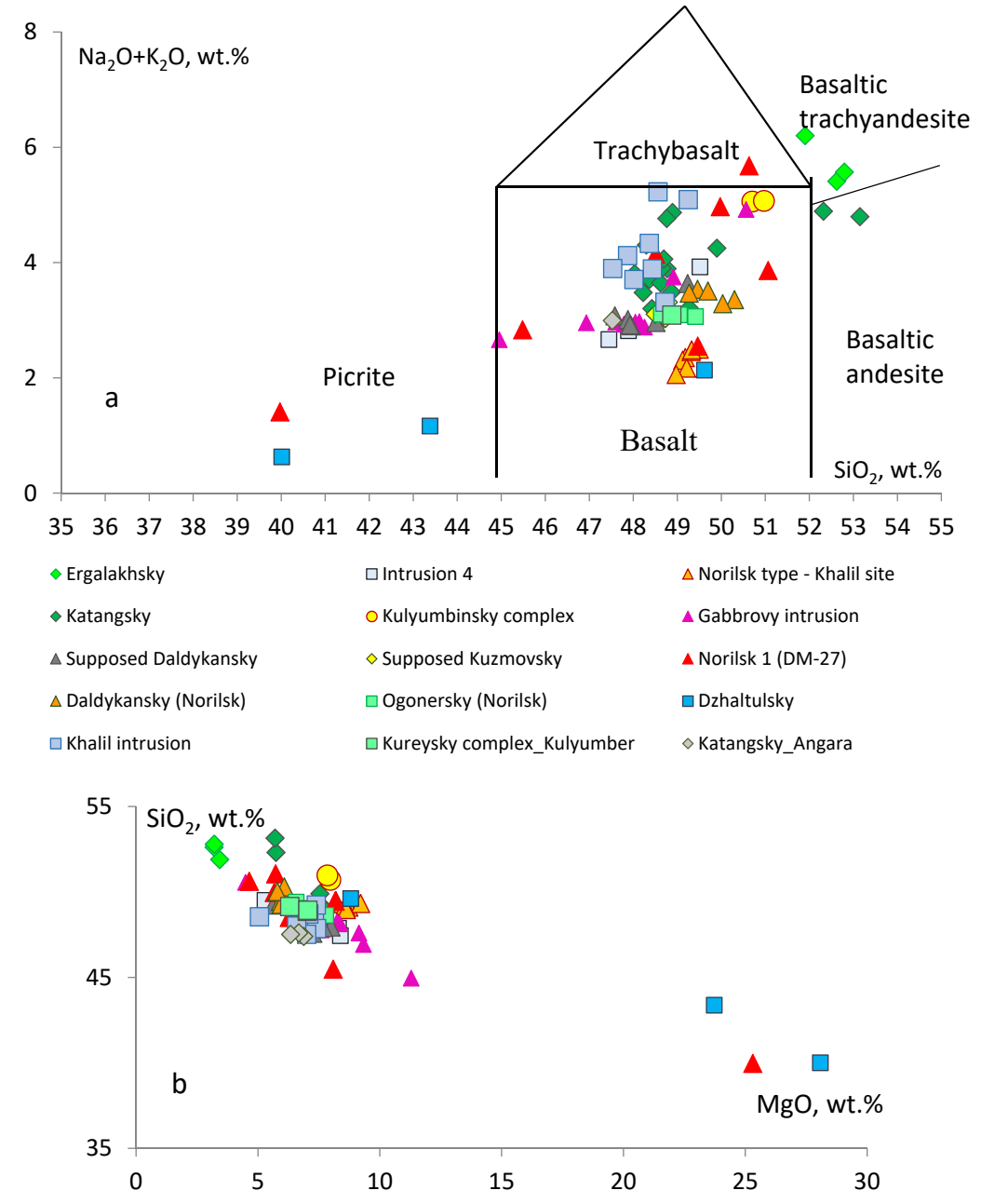

Figure 7. Diagrams $\mathrm{SiO}_{2}$ vs. $\mathrm{Na}_{2} \mathrm{O}+\mathrm{K}_{2} \mathrm{O}$ (a) after [64] and $\mathrm{MgO}$ vs. $\mathrm{SiO}_{2}$ (b) for intrusive rocks. 

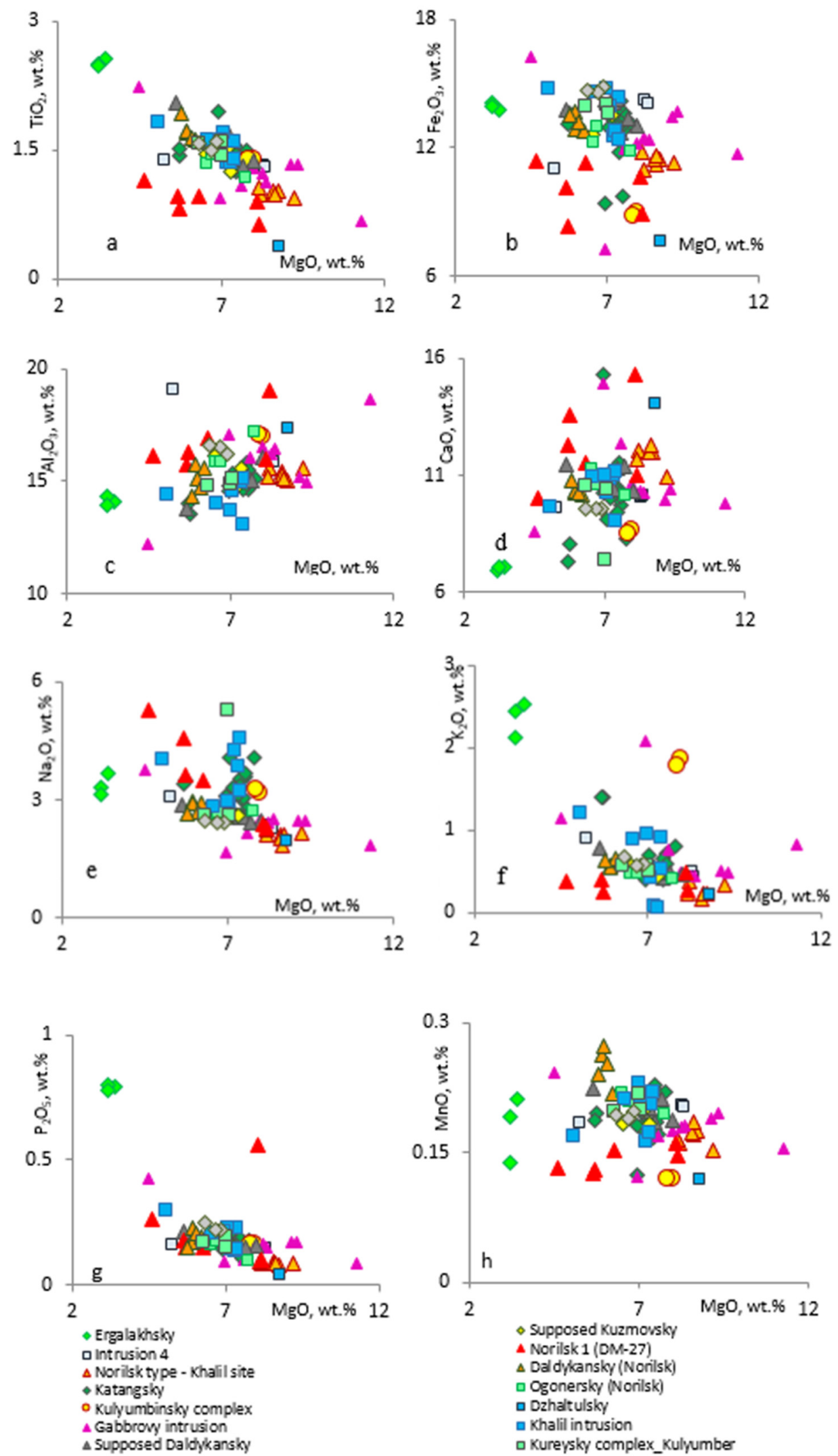

Figure 8. Diagrams $\mathrm{MgO}$ vs. $\mathrm{TiO}_{2}(\mathbf{a}), \mathrm{Fe}_{2} \mathrm{O}_{3}(\mathbf{b}), \mathrm{Al}_{2} \mathrm{O}_{3}(\mathbf{c}), \mathrm{CaO}(\mathbf{d}), \mathrm{Na}_{2} \mathrm{O}(\mathbf{e}), \mathrm{K}_{2} \mathrm{O}(\mathbf{f}), \mathrm{P}_{2} \mathrm{O}_{5}(\mathbf{g})$, and $\mathrm{MnO}$ (h) for intrusive rocks. 
Analysis of behavior of the major elements in the rocks (Figure 8) indicated the presence of two groups of rocks which differed in silica and alkali contents: The Ergalakhsky complex was characterized by the highest concentrations of titanium, potassium, and phosphorus and low silica and calcium in comparison with the other rocks, the points of composition which were plotted close together. According to the $\mathrm{TiO}_{2}$ concentrations, rock compositions from different intrusions formed a single negative trend (Figure 8a) of the titanium depending on the $\mathrm{MgO}$ concentrations, which may be due to the fractional crystallization of a single magma. A similar behavior was observed for $\mathrm{F}_{2} \mathrm{O}_{3}$, although the composition points had a much larger spread (Figure 8b). In both cases, the Norilsk 1 massif had the lower content of these elements, which distinguishes it from other rocks, including the Gabbrovy intrusion, referred to as the Kruglogorsky subcomplex of the Norilsk complex (Figure 9). Thus, the weighted mean $\mathrm{TiO}_{2}$ content in the Gabbrovy intrusion was 1.35 wt.\% (Table 4), while its average mean content in the Norilsk 1 intrusion was $0.87 \mathrm{wt} . \%$, i.e., $<1 \mathrm{wt} . \%$ that is typical of the ore-bearing massifs in the Norilsk area $[18,65,66]$. According to these oxides, this massif could be attributed to the Katangsky complex.
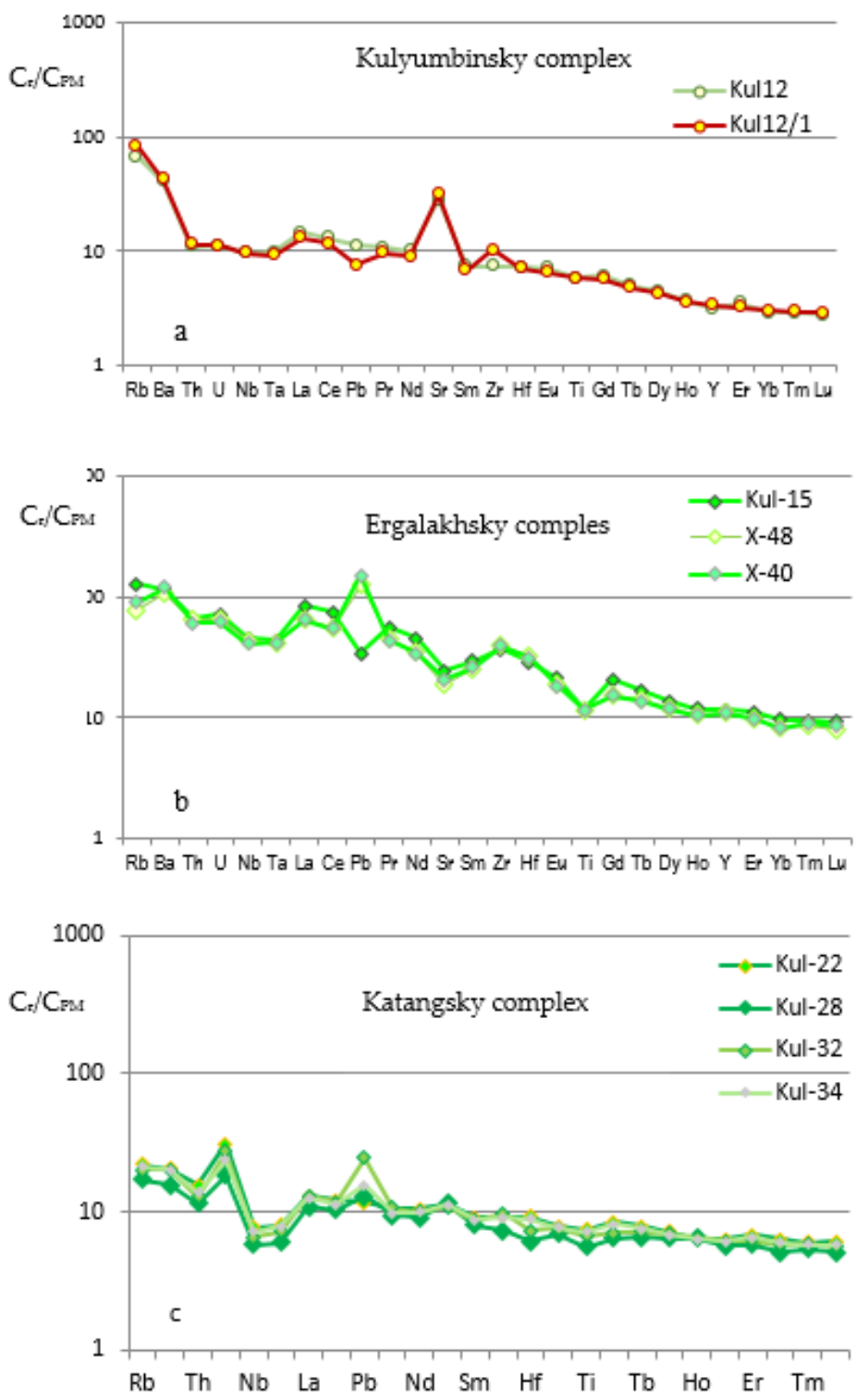

Figure 9. Main types of trace elements' spider diagrams for intrusive rocks of the Kulyumber river valley. Complexes: (a) Kulyumbinsky, (b) Ergalakhsky, (c) Katangsky. 
A slightly lower content of $\mathrm{MnO}$ is also typical of the Norilsk 1 . The $\mathrm{CaO}$ and $\mathrm{Al}_{2} \mathrm{O}_{3}$ demonstrated opposite trends, i.e., they directly correlated with $\mathrm{MgO}$ contents (Figure 8c,d). Points of the $\mathrm{Na}_{2} \mathrm{O}$ concentrations formed two trendlines; one of them was typical of the Norilsk and Kureysky complexes and the second line characterized distribution of this oxide in other rocks (Figure 8e). The highest $\mathrm{K}_{2} \mathrm{O}$ and $\mathrm{P}_{2} \mathrm{O}_{5}$ contents (Figure 8f,g) were typical of the Ergalakhsky and Kulyumbinsky complexes that distinguish them from the other rocks.

For studyng for Kuyumber river it is important valley to compare two complexes that were most widespread: Katangsky and Kureysky. The last named complex was attributed to the Dzhaltulsky massif. We used samples from this massif. The data showed (Figure 8) that there was no difference between the Kureysky intrusive bodies and Katangsky intrusions while the Dzaltulsky massif differed in low $\mathrm{TiO}_{2}$, high $\mathrm{Mg}$, high Al, and low alkalis. The Supposed Kuzmovsky complex was similar to the Katangsky intrusions.

Trace elements. The distribution of trace elements in rocks from different intrusive complexes is shown in a series of spider diagrams (Figure 9), as well as in binary diagrams that reflect the main features of the spectrum topology (Figure 10). There were 3 types of spectra that were fundamentally different from each other: (1) Without $\mathrm{Ta}-\mathrm{Nb}$ and $\mathrm{Pb}$ anomalies characterized by a strong slope of the right part of the spectrum $(\mathrm{Gd} / \mathrm{Yb}) \mathrm{n}=2.1$, samples Kul-12, Kul-12/1 (Figure 9a). We attributed this intrusion to a new Kulyumbinsky complex. (2) with minor Ta-Nb and Sr-negative anomalies, weak positive $\mathrm{Pb}$ anomaly, and steep slope of the right part of the spectrum, $(\mathrm{Gd} / \mathrm{Yb}) \mathrm{n}=1.9-2.1$, $(\mathrm{La} / \mathrm{Sm}) \mathrm{n}=2.5-2.8$, typical of the subalkaline rocks of the Ergalakhsky complex (Figure 9b). (3) With a distinct negative $\mathrm{Ta}-\mathrm{Nb}$ and positive strong $\mathrm{Pb}$ anomalies and with a weak slope of the right part of the spectrum $(\mathrm{Gd} / \mathrm{Yb}) \mathrm{n}=1.3$, typical of most rocks of the Kulyumbinsky and other areas. It included rocks of the Katangsky, Kureysky, Norilsk, Daldykansky, and Oogonersky complexes, and Norilsk 1 and Gabbrovy intrusions. Figure 9c shows patterns for the Katangsky complex. The lowest concentrations of trace elements in this type were typical of the rocks of the Dzhaltulsky massif and picritic gabbro-dolerites from the Norilsk 1 massif, due to the large amount of olivine in the rocks.

Thus, the third group included many intrusions of similar composition in terms of major components but differed in trace elements. These differences reflect the value of anomalies $(\mathrm{Ta}-\mathrm{Nb}, \mathrm{Pb}$, $\mathrm{Sr}$, and $\mathrm{Ti}$ ) and inclination of patterns on the $\mathrm{X}$ axis. Figure 10 demonstrates the difference between the spectra of the rocks from different complexes belonging to the third group. First of all, it was important to compare the rocks of the Norilsk complex from the Norilsk area and the rocks preliminary data attributed to this complex rocks of the Gabbrovy intrusion (Figure 10a,b). Both intrusions had similar spider diagrams and a big difference in trace elements' contents between high-Mg and low-Mg varieties, with total trace element contents changing 2-3 times.

One spectrum in the Gabbrovy intrusion (sample PR-1/224, $\mathrm{MgO}=4.44 \mathrm{wt} . \%$ ) is dramatically enriched in trace elements, i.e. their contents are 3 times higher than the contents in $\mathrm{Mg}$-rich rocks (sample PR- 1/962, MgO = 9.21 wt.\%) and it was characterized by negative $\mathrm{Sr}$ anomaly, which was absent in other patterns. The rocks of similar composition from the Norilsk 1 intrusion had no similar enrichment in trace elements (for example, $\mathrm{DM}-27 / 46, \mathrm{MgO}=4.51 \mathrm{wt} . \%$ ) and had positive $\mathrm{Sr}$ anomaly. We suggest the occurrence of xenolith of any rocks or late sill inside the Gabbrovy intrusion because the behavior of major trace elements did not support the theory of the origin of this sample as a result of fractionation crystallization. The Dzhaltulsky massif (Figure 10e), despite its similarities with the Norilsk 1 and Gabbrovy intrusions, had some differences, i.e., smaller Ta-Nb anomaly and Th and U contents, and strong positive $\mathrm{Pb}$ anomaly (despite the absence of sulfides in these samples).

The rocks from the other intrusive complexes of the third group from the Kulyumber river valley, Norilsk area, and Tunguska syneclise were characterized by different spider diagrams that we demonstrate in Figure 10 for clarity because they have visible differences. The most widespread complexes were Katangsky and Kureysky in the studied area. The first complex comprised sills of gabbro-dolerites and olivine gabbro-dolerites boarding the lavas area of the Siberian trap province. 
These intrusions had thickness varying from one to to 400-500 m. We studied intrusion from this complex outside the Kulyumber river valley, i.e., Padunsky sill in the Angara river valley [29], as well.
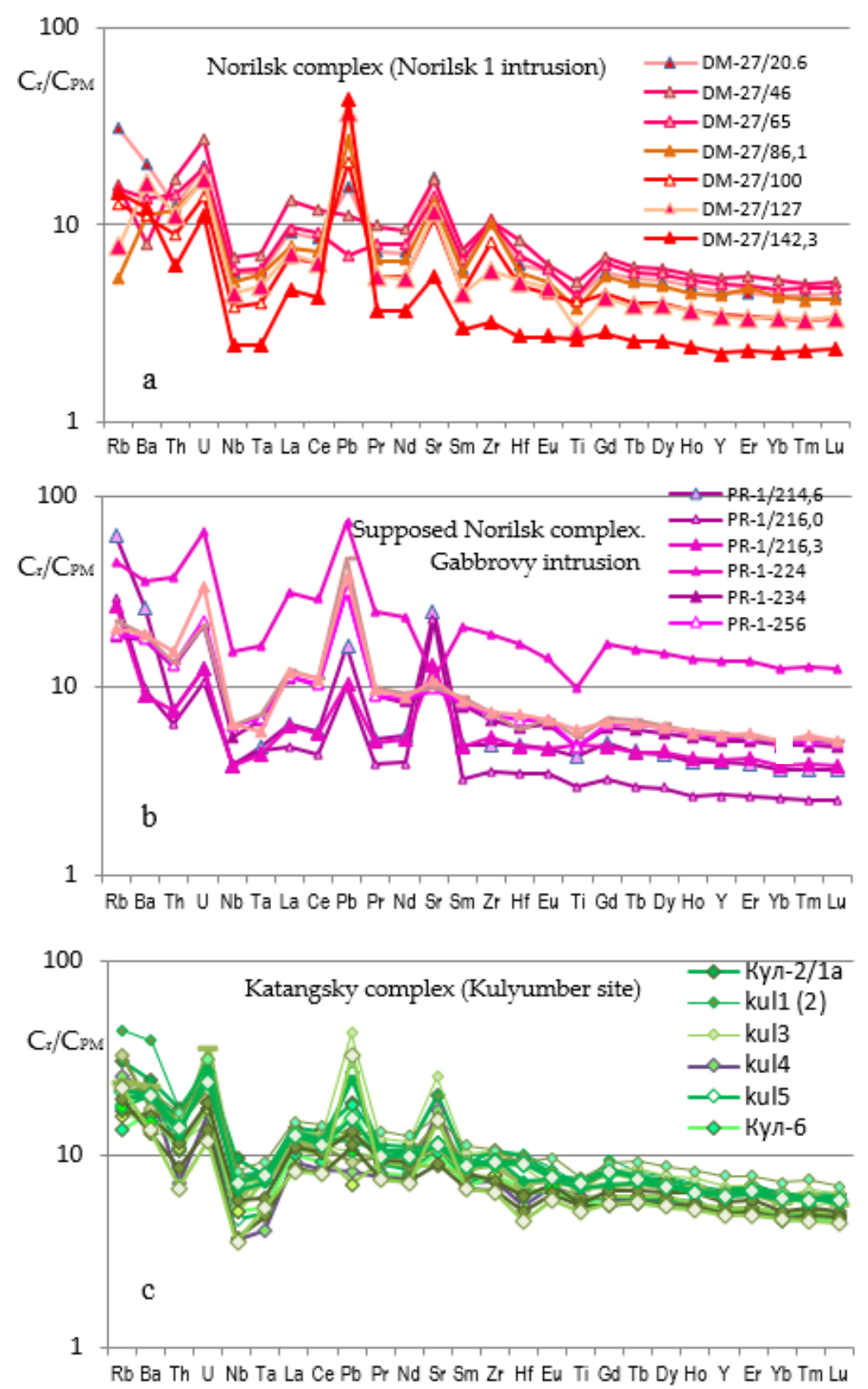

Figure 10. Cont. 

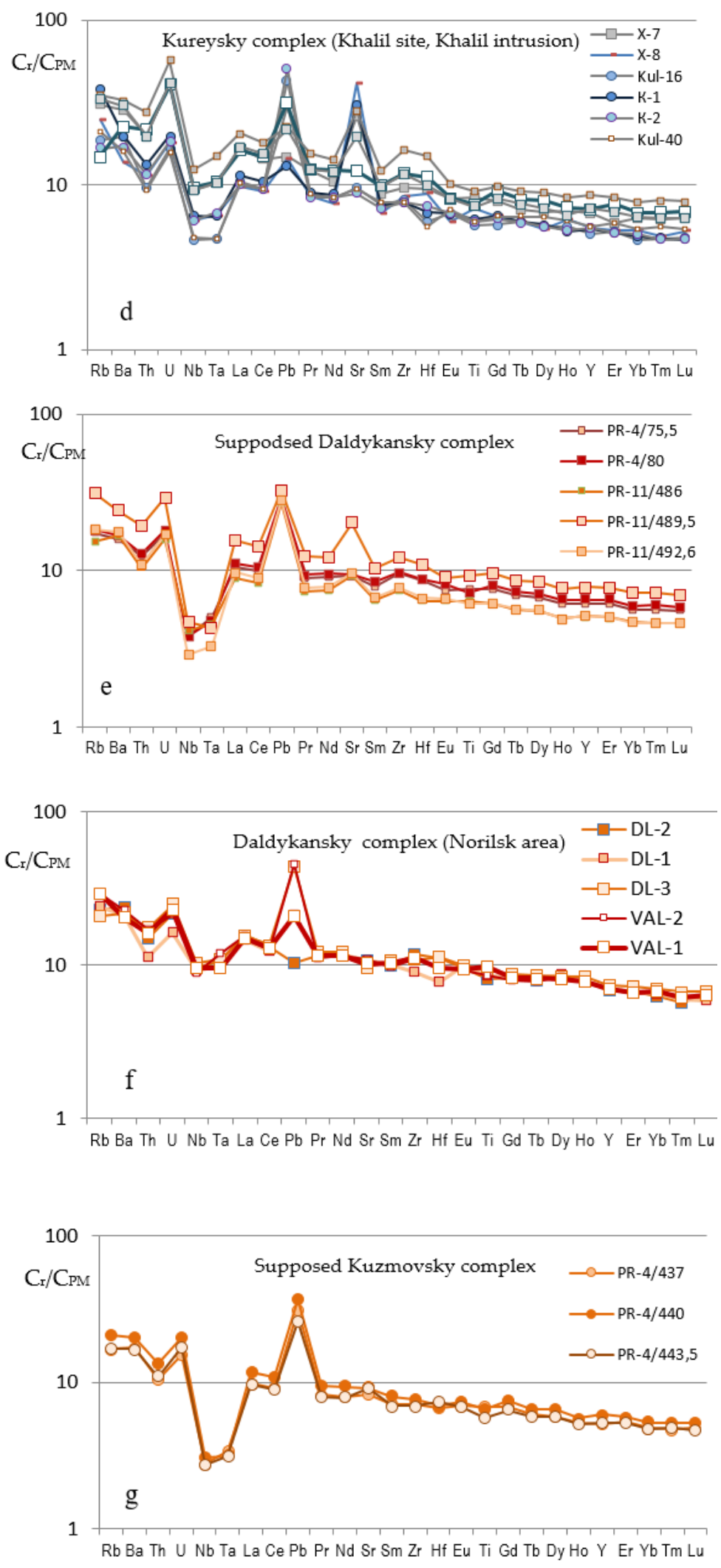

Figure 10. Cont. 

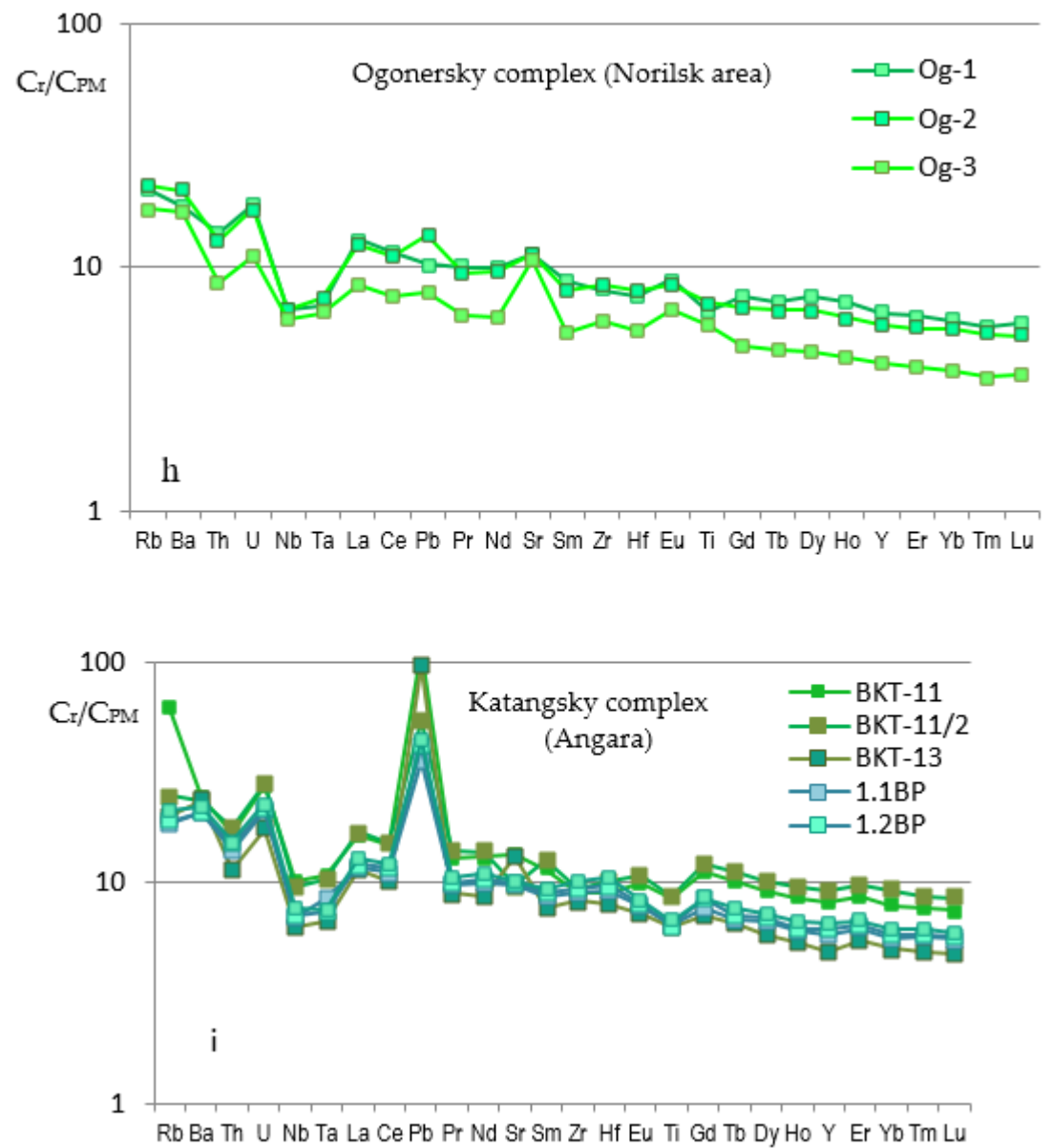

Figure 10. Trace elements' spider diagrams for intrusive rocks of the Siberian platform. Data are in Table 3 and [51]. Complexes: (a) Norilsk, (b) Supposed Norilsk, (c) Katangsky,(d) Kureysky, (e) Supposed Daldykansky, (f) Daldykansky, (g) Supposed Kuzmovsky (h) Ogonersky, (i) Katangsky.

The geochemical features of these intrusions (Figure 10i) were close to the features of the Katangsky (Figure 10c) and Kureysky (Figure 10d) complexes in the Kulyumber site. The rocks of the Daldykansky and Ogonersky complexes located in the Norilsk region differed significantly from those described above (Figure 10f,j). Thus, the rocks preliminarily attributed to these complexes within the Kulyumber area clearly do not belong to them (Figure 10g,h).

A more thorough analysis of the spider diagrams used the ratio of a number of elements that reflected the topology of the spectra (Figure 11). These main relationships included $(\mathrm{La} / \mathrm{Sm}) \mathrm{n},(\mathrm{La} / \mathrm{Yb}) \mathrm{n}$, and $(\mathrm{Gd} / \mathrm{Yb}) \mathrm{n}$, reflecting the overall slope of the spectrum, as well as its individual parts, including the behavior of rare Earth elements in rocks. The $\mathrm{U} / \mathrm{Nb}$ ratio (or $\mathrm{Th} / \mathrm{Nb}$ ) characterizes the value of $\mathrm{Ta}-\mathrm{Nb}$ anomalies. The diagram $(\mathrm{La} / \mathrm{Sm}) \mathrm{n}-(\mathrm{Gd} / \mathrm{Yb}) \mathrm{n}$ (Figure 11a) shows that all intrusions are subdivided into three groups, as noted above. The first two groups included the Ergalakhsky and Kulyumbinsky complexes that were characterized by similar high $(\mathrm{Gd} / \mathrm{Yb}) \mathrm{n}$ ratio $(1.9-2.1)$ and elevated $(\mathrm{La} / \mathrm{Sm}) \mathrm{n}$ value, which was higher for rocks of the Ergalakhsky complex in comparison with this ratio in the Kulyumbinsky complex (2.6-2.9 and 1.9-2.0, respectively). The third group was represented by several intrusive complexes with similar parameters. But these intrusions showed certain differences among themselves in the $(\mathrm{U} / \mathrm{Nb}) \mathrm{n}$ ratio (Figure $11 \mathrm{~b}, \mathrm{c}$ ), and a significant difference from the rocks of the first and second groups (Kulyumbinsky and Eergalakhsky complexes), characterized by minimal values of this ratio. 

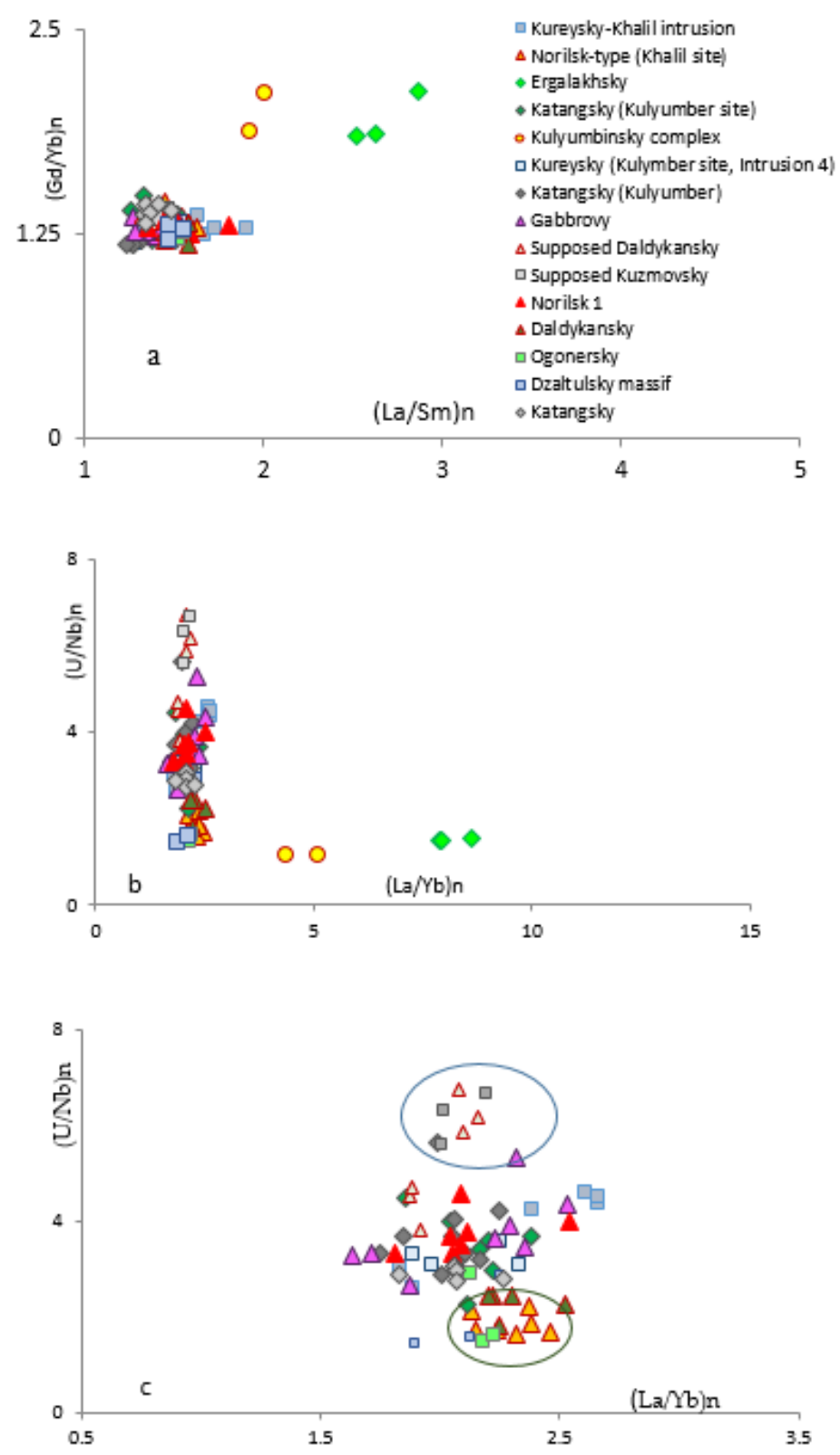

Figure 11. Diagrams $(\mathrm{La} / \mathrm{Sm}) \mathrm{n}$ vs. $(\mathrm{Gd} / \mathrm{Yb}) \mathrm{n}(\mathbf{a})$ and $(\mathrm{U} / \mathrm{Nb}) n$ vs. $(\mathrm{La} / \mathrm{Yb}) n(\mathbf{b}, \mathbf{c})$ for intrusive rocks. The symbols on all three diagrams are the same as on (a).

The rocks of the third group formed a wide range of compositions, varying in $(\mathrm{U} / \mathrm{Nb}) \mathrm{n}$ ratio. A more detailed examination of these rocks (Figure 11c) indicated that there were intrusions with high, low, and intermediate $(\mathrm{U} / \mathrm{Nb}) \mathrm{n}$ ratios, reflecting the most pronounced $\mathrm{Ta}-\mathrm{Nb}$ anomaly, the minimum pronounced, and ordinary apparent. The highest $(\mathrm{U} / \mathrm{Nb}) \mathrm{n}$ ratio was typical of the Supposed Kuzmovsky and Supposed Daldykansky complexes penetrated by PR-4 and PR-11 boreholes. One sample from the Gabbrovy intrusion (PR-1/224) has a high $(\mathrm{U} / \mathrm{Nb})$ n ratio, as well, and occurred in the same field which supports its xenogenous origin in comparison with the other samples from this intrusion. The lowest $(\mathrm{U} / \mathrm{Nb}) \mathrm{n}$ ratio characterized the Daldykansky and Ogonersky complexes (Norilsk area), Norilsk-type intrusion in the Khalil site, and Dzaltulsky massif. The rock compositions of the Norilsk 1 intrusion, Gabbrovy intrusion, and Katangsky and Kureysky complexes formed a single field, where points of the Khalil intrusion occurred at the edge of this field. 


\subsubsection{Isotope Composition of Intrusive Rocks}

To characterize the initial isotope composition for the main intrusive complexes of the Kuluymber river valley, we analyzed $\mathrm{Sr}, \mathrm{Nd}$, and $\mathrm{Pb}$ isotope systems of representative samples from the Ergalakhsky, Kulyumbinsky, Kureysky (Khalil intrusion), Supposed Norilsk-type (the Khalil site), and Gabbrovy intrusion. The obtained results for 11 whole-rock samples of mafic intrusions and one of argillite from host Tunguska Group are presented in Table 5 and in Figures 12 and 13. The variations in element contents were prominent for Rb and Sr: From 0.8 up to 50 and from 170 up to $660 \mathrm{ppm}$, correspondingly, and were pretty restricted for Sm and Nd: From 2.4 up to 3.6, and from 8.4 up to 12.8 ppm, respectively, excluding subalkaline rocks of the Ergalakhsky complex and sulfide-metasomatized rocks of the Katangsky complex, which are enriched in REE by $2-4$ times in comparison with other samples.

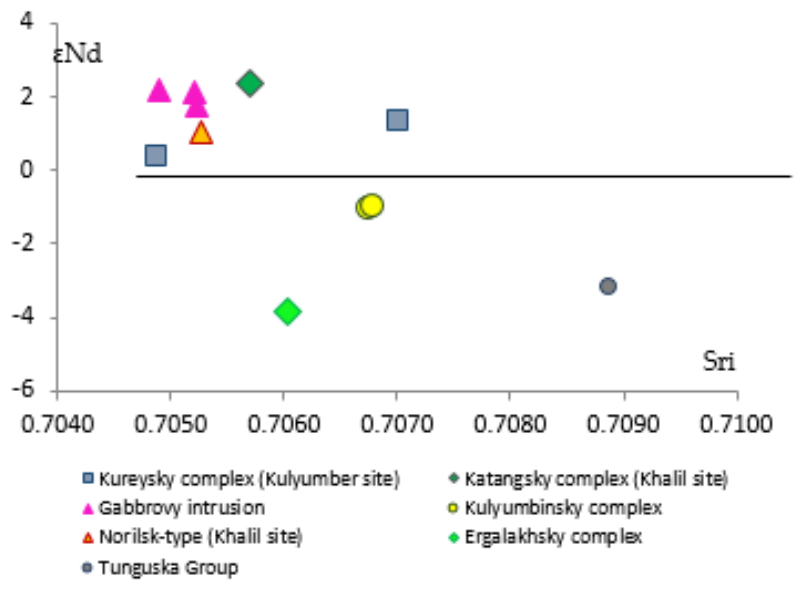

Figure 12. Initial isotope signatures for dolerites and basalts from intrusive complexes of the Kulyumber river valley in comparison with rock composition of the same complexes from Norilsk-type locality.

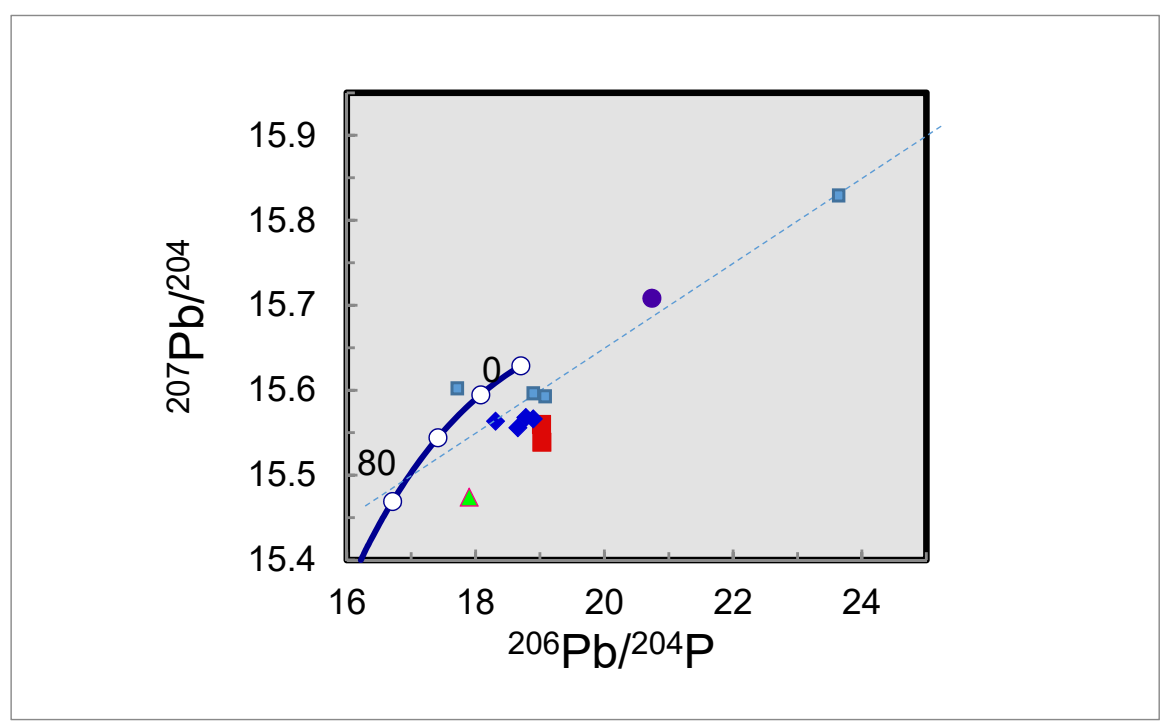

Figure 13. $\mathrm{Pb}-\mathrm{Pb}$ isotope diagram for measured $\mathrm{Pb}$ isotope data for whole-rock samples of intrusive complexes of the Kulyumber river valley. Green triangle, subalkaline dolerite from Ergalakhsky complex; red squares, Kulyumbinsky complex; blue diamonds, Supposed Norilsk-type Gabbrovy intrusion; blue rectangles, Kureysky complex; magenta circle, argillite of the Tunguska Group; dashed line corresponds to isochron with the age 251 Ma on Kureysky complex samples, including sulfide mineralized sample X-15-2. 
Table 5. Isotope composition of the intrusive whole rocks from the Kulyumber river valley.

\begin{tabular}{|c|c|c|c|c|c|c|c|c|c|}
\hline No. & Sample & $\mathrm{Rb}, \mathrm{ppm}$ & \multicolumn{2}{|c|}{ Sr, ppm } & ${ }^{87} \mathrm{Rb} /{ }^{86} \mathrm{Sr}$ & \multicolumn{2}{|c|}{${ }^{87} \mathrm{Sr} /{ }^{86} \mathrm{Sr} \pm 2 \sigma$} & Sri & $\varepsilon \mathrm{Sr}$ \\
\hline 1 & $\mathrm{X}-40$ & 55.1 & \multicolumn{2}{|r|}{388} & 0.41110 & \multicolumn{2}{|c|}{$0.707496 \pm 6$} & 0.706034 & 25.9 \\
\hline 2 & Kul-12 & 52.1 & \multicolumn{2}{|r|}{643} & 0.23448 & \multicolumn{2}{|c|}{$0.707582 \pm 6$} & 0.706745 & 36.0 \\
\hline 3 & Kul-12/1 & 52.6 & \multicolumn{2}{|r|}{647} & 0.23536 & \multicolumn{2}{|c|}{$0.707613 \pm 4$} & 0.706773 & 36.4 \\
\hline 4 & K-1 & 23.1 & \multicolumn{2}{|r|}{618} & 0.10819 & \multicolumn{2}{|c|}{$0.707404 \pm 14$} & 0.707019 & 39.9 \\
\hline 5 & K-2 & 10.0 & \multicolumn{2}{|r|}{201} & 0.14361 & \multicolumn{2}{|c|}{$0.705375 \pm 7$} & 0.704864 & 9.30 \\
\hline 6 & $X-6$ & 0.84 & \multicolumn{2}{|r|}{175} & 0.01388 & \multicolumn{2}{|c|}{$0.705743 \pm 6$} & 0.705694 & 21.1 \\
\hline 7 & PR-1/262 & 13.1 & \multicolumn{2}{|r|}{208} & 0.18165 & \multicolumn{2}{|c|}{$0.705868 \pm 11$} & 0.705222 & 14.4 \\
\hline 8 & PR-1/234 & 11.0 & \multicolumn{2}{|r|}{200} & 0.15897 & \multicolumn{2}{|c|}{$0.705453 \pm 8$} & 0.704887 & 9.62 \\
\hline 9 & PR-1/266 & 11.7 & \multicolumn{2}{|r|}{223} & 0.15232 & \multicolumn{2}{|c|}{$0.705748 \pm 7$} & 0.705206 & 14.2 \\
\hline 10 & $\mathrm{X}-32$ & 8.36 & \multicolumn{2}{|r|}{218} & 0.11071 & \multicolumn{2}{|c|}{$0.705669 \pm 5$} & 0.705275 & 15.1 \\
\hline 11 & $X-15-2$ & 15.6 & \multicolumn{2}{|r|}{666} & 0.06796 & \multicolumn{2}{|c|}{$0.708479 \pm 6$} & 0.708237 & 57.2 \\
\hline 12 & $X-47-2$ & 38.6 & \multicolumn{2}{|r|}{178} & 0.62973 & \multicolumn{2}{|c|}{$0.711116 \pm 12$} & 0.708876 & 66.3 \\
\hline No. & Sm, ppm & $\mathrm{Nd}, \mathrm{ppm}$ & ${ }^{147} \mathrm{Sm} /{ }^{144} \mathrm{Nd}$ & ${ }^{143} \mathrm{Nd} /{ }^{144} \mathrm{Nd} \pm 2 \sigma$ & Ndi & $\varepsilon \mathrm{Nd}$ & ${ }^{206} \mathrm{~Pb} /{ }^{204} \mathrm{~Pb} \pm 2 \sigma$ & ${ }^{207} \mathrm{~Pb} /{ }^{204} \mathrm{~Pb} \pm 2 \sigma$ & ${ }^{208} \mathrm{~Pb} /{ }^{204} \mathrm{~Pb} \pm 2 \sigma$ \\
\hline 1 & 11.6 & 55.4 & 0.12612 & $0.512325 \pm 2$ & 0.512118 & -3.84 & $17.9002 \pm 5$ & $15.4741 \pm 7$ & $37.9470 \pm 23$ \\
\hline 2 & 3.14 & 12.7 & 0.14942 & $0.512508 \pm 3$ & 0.512263 & -1.02 & $19.0257 \pm 102$ & $15.5595 \pm 68$ & $39.2923 \pm 181$ \\
\hline 3 & 3.18 & 12.8 & 0.15011 & $0.512512 \pm 7$ & 0.512265 & -0.97 & $19.0320 \pm 17$ & $15.5387 \pm 15$ & $39.2027 \pm 42$ \\
\hline 4 & 3.33 & 12.1 & 0.16623 & $0.512656 \pm 5$ & 0.512384 & 1.32 & $19.4365 \pm 12$ & $15.6122 \pm 10$ & $39.0764 \pm 27$ \\
\hline 5 & 3.32 & 11.8 & 0.16968 & $0.512614 \pm 6$ & 0.512337 & 0.40 & $18.2287 \pm 7$ & $15.6180 \pm 7$ & $38.0641 \pm 19$ \\
\hline 6 & 3.40 & 11.6 & 0.17796 & $0.512727 \pm 2$ & 0.512435 & 2.34 & $19.2871 \pm 8$ & $15.6147 \pm 7$ & $39.1369 \pm 21$ \\
\hline 7 & 3.64 & 13.1 & 0.16820 & $0.512680 \pm 2$ & 0.512405 & 1.73 & $18.6576 \pm 9$ & $15.5558 \pm 8$ & $38.1920 \pm 20$ \\
\hline 8 & 3.25 & 11.6 & 0.16889 & $0.512704 \pm 2$ & 0.512428 & 2.19 & $18.7802 \pm 20$ & $15.5679 \pm 17$ & $38.3141 \pm 43$ \\
\hline 9 & 3.25 & 11.6 & 0.16970 & $0.512702 \pm 2$ & 0.512424 & 2.12 & $18.8960 \pm 7$ & $15.5662 \pm 6$ & $38.2286 \pm 16$ \\
\hline 10 & 2.41 & 8.37 & 0.17377 & $0.512652 \pm 5$ & 0.512367 & 1.01 & $18.3089 \pm 8$ & $15.5636 \pm 8$ & $38.1935 \pm 24$ \\
\hline 11 & 6.48 & 30.3 & 0.12915 & $0.512388 \pm 6$ & 0.512176 & -2.70 & $23.5360 \pm 9$ & $15.8283 \pm 7$ & $40.7615 \pm 18$ \\
\hline 12 & 5.37 & 27.8 & 0.11677 & $0.512345 \pm 3$ & 0.512153 & -3.16 & $20.7381 \pm 6$ & $15.7081 \pm 5$ & $40.4289 \pm 19$ \\
\hline
\end{tabular}

Note. Nos. 1-11 correspond to the analysis of samples from intrusive complexes: 1, Ergalakhsky; 2, 3, Kulyumbinsky; 4-6, Kureysky; 7-10, Norilsk; 7-9, Kruglogorsky subcomplex; 10, Norilsk-type intrusion; 11, metasomatized gabbro-dolerite; 12, argillite from the Tunguska Group. Ndi, Sri, $\varepsilon$ Nd, and Sri-the initial isotopic compositions of the studied samples (in absolute or relative values- $\varepsilon$ ) were calculated for the age of $250 \mathrm{Ma}$ and sample 12 for $295 \mathrm{Ma}$. Color means different rock groups. 
Despite these variations, the initial isotopic signatures of the samples studied were characterized by high consistency inside the intrusive complexes and, at the same time, they differed significantly between these complexes. Thus, the rocks of Ergalakhsky complex had an initial strontium ratio value (Sri) at 0.7060 and lowest epsilon neodymium value, -3.8 , while the rocks of the Kulyumbinsky complex were characterized by Sri 0.7067 and epsilon neodymium at -1.0, and the Gabbrovy intrusion had the lowest Sri values, from 0.7049 up to 0.7052 , and the highest epsilon neodymium, from +1.0 up to +2.2 (Figure 12). While the metasomatized sulfide-containing basalt of the Katangsky complex had initial isotopic parameters that were almost identical to the sedimentary rocks of the Tunguska Group, namely, Sri 0.7082 for basalt (X-15-2) versus 0.7088 for argillite (X-47-2) and $\varepsilon N d-2.7$ for basalt and -3.2 for argillite. The studied samples of the Kureysky complex had the most variable isotopic characteristics, so the initial isotopic composition of strontium varied from the lowest value among the studied samples, 0.7048 , to one of the highest, -0.7070 . In this case, the isotopic composition of neodymium varied within 2 units of epsilon, from +0.4 to +2.3 . Perhaps these variations, which are atypical for other complexes, are explained by a combination of separate intrusions genetically belonging to other intrusive complexes (e.g., Intrusion 4 and Khalil intrusions).

It should be noted that the initial isotopic characteristics of intrusive complexes from the Kuluymber river valley were close to those obtained previously for the complexes of the same name sampled in other areas of the Norilsk region. Thus, the subalkaline trachydolerites of the Ergalakhsky complex from the northern and southern parts of the Norilsk district had neodymium composition nearly the same: $\varepsilon N d$ within -3.8 and -4.2 [67-69] and only the initial strontium isotopic composition, 0.7064-0.7075, was slightly higher than the value we measured in sample X-40.

Mafic rocks of the Kruglogorsky subcomplex of the Norilsk intrusive complex were characterized by weakly positive values of neodymium epsilon, from +1.5 to +2.2 , and strongly varying strontium isotopic compositions, from 0.7062 to 0.7084 [67], while the Supposed Kruglogorsky subcomplex of the Norilsk complex in the Kulyumber river valley (Gabbrovy intrusion) had a comparable isotopic neodymium composition (from +1.0 to +2.2 ) and significantly less radiogenic compositions of strontium, $0.7048-0.7052$, which apparently is associated with steadily low rubidium content in these rocks. Thus, at first glance, the initial isotopic characteristics of the Gabbrovy intrusion rocks did not contradict the assumption that they belong to the Norilsk complex (Kruglogorsky subcomplex).

Alternatively, a separate local mantle source could exist for the Kulyumbinsky intrusive complex, which differed in composition both from the sources of the Norilsk type and from the Ergalakhsky subalkaline type of intrusion. The fact that it was rather a separate mantle source is also suggested by the specificity of the lead isotopic composition (Figure 13). The rocks of the Kulyumbinsky complex occupied an intermediate position between the Kureysky and Ergalakhsky rocks on the isotopic diagram ${ }^{206} \mathrm{~Pb} /{ }^{204} \mathrm{~Pb}-{ }^{207} \mathrm{~Pb} /{ }^{204} \mathrm{~Pb}$, but presented data were limited only by two samples of rocks of the Kulyumbinsky complex, which were almost identical in composition, and we do not know the real scatter of isotopic data for this complex. Nevertheless, there is no doubt over the influence of the host rocks during the formation of sulfide-containing metasomatite upon gabbrodolerite (sample X-15-2). For example, in the $\mathrm{Pb}-\mathrm{Pb}$ isotope space, it was possible to construct an isochron line on the samples of the Kureysky complex through a sulfide-containing dolerite of sample X-15-2, corresponding to an age of $250 \mathrm{Ma}$, and the argillite data point also fell on this line (Figure 13).

\section{Discussion}

\subsection{Tectonic Structure}

In 1936 V. Sobolev [1] performed the first detailed petrographic description of igneous rocks of the Siberian traps province. This publication defined the main directions of intraplate magmatism study for many years. These lines of research included: (1) Study of regional petrochemical provinces within the Siberian platform, (2) determination of the composition of the initial magmas, (3) analysis of magma intra-chamber differentiations, and (4) ore deposits' formation modeling. 
The analysis of the different petrographic provinces is given in many works of V. Zolotukhin with co-authors [3,5,7]. The following structural zones were identified: Prieniseysky, Putorana, Tunguska syncline, Predtaimyrsky, and South Taimyr on the basis of the State Geological Survey (1:200,000 scale) and the analytical data were obtained by the authors on the composition of magmatic rocks within the Siberian platform. Magmatism within these zones is controlled by (1) Early Mesozoic swell-like uplifts that underwent inversion in the Late Mesozoic and is associated with ancient (Riphean) rifts (Khantaysko-Rybninsky, Kureysko-Letninsky, Pyasinsky), (2) ancient linear structures that did not undergo inversion (Predtaymyrsky), (3) ancient aulacogens regenerated in the pre-Mesozoic period (Vilyuysky, Udzhinsky), and (4) early Mesozoic troughs (within the Tunguska syneclise) that did not undergo inversion.

The Prieniseysky zone is the most important tectonic structure which is characterized by a diverse composition of magmatic rocks and high concentration of $\mathrm{Cu}-\mathrm{Ni}$ deposits. It includes [70] Khantaika-Rybinskoe and Kureysky-Letninsky zones. The latter is a part of a large submeridional tectonic structure in the border zone of the Siberian craton comprising three sublatitude branches, one of which is located within the Kulyumber river valley.

This structure was identified on the basis of the gravimetric mapping of 1:200,000 scale and airmagnetic data of 1:100,000 scale [51] and named as the Norilsk-Igarka paleorift earlier [2,71].

\subsection{Magmatic Rocks}

\section{Subdivision of Intrusive Rocks}

The study of igneous rocks of the Kulyumber river valley and adjacent territories was carried out in the early 1960s in the course of geological mapping. On the basis of the internal structure of intrusive bodies and the distribution of the main components, the Ergalakhsky complex of high alkalinity and four complexes of moderate alkalinity were identified. The latter include (1) Katangsky complex, comprising undifferentiated or weakly differentiated small- to medium-grained intrusions stable in composition (gabbro-dolerites with 6-7 wt.\% MgO) and widespread in the frame of the lava field of the Siberian trap province; (2) Kureysky complex, combining differentiated large intrusive bodies of elevated $\mathrm{MgO}$ (8-9 wt.\%) with troktolite lenses in olivine gabbro-dolerites; (3) Kuzmovsky complex, integrateing weakly differentiated gabbro-dolerite intrusions with ferrogabbro and granophyres in the upper part of intrusive bodies; and (4) Norilsk complex, which includes highly differentiated intrusions with average $\mathrm{MgO}-10-12 \mathrm{wt} . \%$.

Our geochemical data indicated the presence of four different groups of igneous rocks within the Kulyumber river valley, which includes effusive and intrusive facies:

(1) Mafic and ultramafic volcanic rocks of high and moderate magnesium ( $\mathrm{MgO}=7-12 \mathrm{wt} . \%)$ with elevated $\mathrm{TiO}_{2}$ (1.2-1.4 wt.\%) characterized by the absence (or very weakly presence) of Ta- $\mathrm{Nb}$-negative and $\mathrm{Pb}$-positive anomalies and the following geochemical parameters: $(\mathrm{La} / \mathrm{Sm}) \mathrm{n}=1.45,(\mathrm{Gd} / \mathrm{Yb}) \mathrm{n}=1.89$, ${ }^{87} \mathrm{Sr} /{ }^{86} \mathrm{Sr}=0.70544, \varepsilon \mathrm{Nd}=+3.9$. These rocks had a mantle origin [42] with garnet in their source and belong to the Gudchikhinsky formation.

(2) Mafic intrusive rocks of moderate magnesium $(\mathrm{MgO}=7.7 \mathrm{wt} . \%)$ with $\mathrm{TiO}_{2}=1.36$ wt. $\%$ without $\mathrm{Ta}-\mathrm{Nb}$ and $\mathrm{Pb}$ anomalies with $(\mathrm{La} / \mathrm{Sm}) \mathrm{n}=2.00,(\mathrm{Gd} / \mathrm{Yb}) \mathrm{n}=2.00,{ }^{86} \mathrm{Sr} /{ }^{87} \mathrm{Sr}=0.70675$, and preliminarily attributed to the Gudchikhinsky formation [60] but differing in reduced values of $\varepsilon \mathrm{Nd}=-1.0$ (in comparison with +4 for the Gudchikhinsky rocks). On the basis of these data, we identified a new intrusive complex named Kulyumbinsky (Figure 3).

(3) Subalkaline volcanic rocks of low magnesium $(\mathrm{MgO}=3.5-5.1 \mathrm{wt} . \%)$ with high $\mathrm{TiO}_{2}(1.5-1.8$ wt.\%) of the Syverminsky formation and intrusive rocks $\left(\mathrm{MgO}=3.2 ; \mathrm{TiO}_{2}=2.4 \mathrm{wt}\right.$.\%) of the Ergalakhsky complex with a small Ta- $\mathrm{Nb}$ anomaly. Volcanic and intrusive rocks are characterized by $(\mathrm{La} / \mathrm{Sm}) \mathrm{n}=2.3-3.5$ and $2.5-2.8$, and $(\mathrm{Gd} / \mathrm{Yb}) \mathrm{n}=1.5-1.8$ and $1.9-2.0,{ }^{87} \mathrm{Sr} /{ }^{86} \mathrm{Sr}=0.70754-0.70824$ and $0.706034, \varepsilon \mathrm{Nd}=-3.1$ and -3 , respectively. They are from crustal source enriched in garnet. 
(4) Mafic intrusive rocks of moderate and increased magnesium ( $\mathrm{MgO}=6-9$ wt.\%) with strong negative $\mathrm{Ta}-\mathrm{Nb}$ and positive $\mathrm{Pb}$ anomalies. Wide range of ratios are typical of these rocks $(\mathrm{La} / \mathrm{Sm}) \mathrm{n}=1.3-1.4,(\mathrm{Gd} / \mathrm{Yb}) \mathrm{n}=1.2-1.5,{ }^{87} \mathrm{Sr} /{ }^{86} \mathrm{Sr}=0.70489-0.70702, \varepsilon \mathrm{Nd}=0.4-2.2$. This group includes different rocks, attributed to the Katangsky, Kuzmovsky, and Norilsk complexes. Their geochemical features are given in Table 6.

The special relationships between studied intrusions of these complexes are unclear due to an absence of geological boundaries between them caused by their separated positions. The legend to the State Geological map [53] confirms the earlier formation of the Katangsky complex in comparison with the Kureysky because several dykes preliminarily attributed to the last complex cut the Dzhaltulsky massif and basalts of the Mokulaevsky formation. Paleomagnetic data [60] show evidence that intrusions in the Kulyumber site (mainly Katangsky complex) are coeval with the basalts of the Mokulaevsky-Kharaelakhsky formations. This conclusion correlates with geochemical data showing $\mathrm{TiO}_{2}$ increasing in basalts during the evolution of platform magmatism in western Siberia (from Tuklonsky to Samoedsky formation [72]). Similar $\mathrm{TiO}_{2}$ behavior occurred in the intrusive rocks: The earliest intrusions belong to the Norilsk complex $\left(\mathrm{TiO}_{2}<1 \mathrm{wt} . \%\right)$, which cuts the Lower Nadezhdinsky formation and include the Dzhaltulsky massif $\left(0.8 \mathrm{wt} . \% \mathrm{TiO}_{2}\right)$. The latest intrusions are the Katangsky and Kureysky complexes, very close (1.4-1.5 wt.\% $\mathrm{TiO}_{2}$ ) to the Kharaelakhsky formation, which cuts the Mokulaevsky formation.

The complexity of the last group required separate consideration of studied rocks. First, the rocks of the Supposed Daldykansky and Supposed Kuzmovsky complexes penetrated by boreholes PR-4 and PR-11 were similar in composition and did not actually correspond to the reference Daldykansky and Kuzmovsky complexes in the Norilsk area (Figure 10e-g): They were characterized by low titanium and pronounced Ta-Nb-negative anomaly. They correspond to the rocks of the Katangsky complex.

Many studied intrusive bodies belonged to the Katangsky complex. They had very similar fabric and composition and there was no doubt in regards to their attribution. Several intrusive bodies within the Khalil and Kulyumber areas were described as massifs of the Kureysky complex (Khalil intrusion, Intrusion 4 in the Kulyumber site, sample Kul-40) on the basis of coarse-grained texture and elevated $\mathrm{MgO}$ content (Table 5). Nevertheless, two main intrusions, Khalil and Intrusion 4, were not identical. They have some differences in $\varepsilon \mathrm{Nd}$ (2.34 and 1.3). Intrusion 4 in the Kulyumber site was very similar to the Gabbrovy intrusion (borehole PR-1) in major and trace elements as well isotope composition.

The Gabbrovy intrusion is of a particular interest in the Kulyumber river valley due to its sulfide mineralization. It is considered as a promising object for the prospecting of Cu-Ni-rich ore. It was attributed to the Kruglogorsky subcomplex of the Norilsk complex on the basis of the occurrence of leucogabbro horizon in its upper zone (Figure 13). Sill-like bodies of this subcomplex occur in the main ore junctions of the Norilsk region, the Norilsk and Talnakh, along with ore-bearing intrusions. This combination raises the question of the genetic relationship between the Norilsk and Kruglogorsky subcomplexes. This question was first formulated by Likhachev in 1965 [73]. Leucocratic rocks were also present not only in the Kruglogorsky sills but in ore-bearing intrusions as well. These rocks were represented by two varieties: Coarse-grained panidiomorphic rocks, often with massive or porphyric texture, and rocks with ataxitic structure [73-76]. In both cases, the rocks were enriched in plagioclase, the amount and morphology of which varied. The first variety consisted of short-prismatic plagioclase crystals (70-90 vol.\%) and represented anorthosites (with $\mathrm{An}_{65-90}$ ). The second variety is comprised of more dark-color minerals (pyroxene and olivine, 20-40 vol.\%) that are irregularly distributed in rocks and form segregations in $2-10 \mathrm{~cm}$. They were named taxitic gabbro-dolerites. Usually these rocks are described together as leucogabbro. Meanwhile, they had different positions within intrusive bodies and different origins. 
Table 6. Comparison of different intrusions from different complexes.

\begin{tabular}{|c|c|c|c|c|c|c|c|c|c|c|c|}
\hline No. & Complex & Site, Intrusion & Sample $(n)$ & $\mathrm{MgO}$ & $\mathrm{TiO}_{2}$ & $\mathrm{Na}_{2} \mathrm{O}+\mathrm{K}_{2} \mathrm{O}$ & $(\mathrm{La} / \mathrm{Sm}) \mathrm{n}$ & $(G d / Y b) n$ & $(\mathrm{U} / \mathrm{Nb}) \mathrm{n}$ & $87 \mathrm{Sr} / 86 \mathrm{Sr}$ & $\varepsilon \mathbf{N d}$ \\
\hline 1 & Ergalakhsky & Khalil & $X-40$ & 3.12 & 2.41 & 6.09 & 2.52 & 1.85 & 1.51 & 0.706034 & -3.8 \\
\hline 2 & & Norilsk area & $n=3[67]$ & & & & & & & 0.706459 & -4.0 \\
\hline 3 & & South Norilsk & $n=1[69]$ & 2.83 & 3.33 & 6.63 & 2.61 & 1.77 & 1.43 & 0.70758 & -3.8 \\
\hline 4 & Kulyumbinsky & $\begin{array}{l}\text { Kulyumber } \\
\text { Intrusion } 5\end{array}$ & Kul-12 & 7.72 & 1.36 & 4.9 & 2.00 & 2.12 & 1.17 & $\begin{array}{l}0.706745 \\
0.706773\end{array}$ & $\begin{array}{l}-1.0 \\
-1.0\end{array}$ \\
\hline 6 & Daldykansky & Norilsk area & $n=5[51]$ & 6.00 & 1.72 & 3.43 & 1.51 & 1.25 & 2.26 & 0.705768 & 3.1 \\
\hline 7 & Kureysky & $\begin{array}{l}\text { Kulyumber } \\
\text { Intrusion } 4\end{array}$ & $\mathrm{~K}-2$ & 8.3 & 1.29 & 2.64 & 1.40 & 1.23 & 3.04 & 0.704864 & 0.4 \\
\hline 8 & & Khalil site, Khalil & $x-6$ & 6.99 & 1.31 & 4.21 & 1.35 & 1.25 & 2.58 & 0.705694 & 1.0 \\
\hline 9 & Norilsk-type & $\begin{array}{l}\text { Khalil site } \\
\text { Intrusion } 2\end{array}$ & $x-32$ & 8.55 & 1.00 & 2.26 & 1.62 & 1.29 & 1.68 & 0.705275 & 1.0 \\
\hline 10 & Kruglogorsky & Kulyumber & PR-1/262.2 & 9.05 & 1.31 & 2.94 & 1.39 & 1.33 & 3.46 & 0.705222 & 1.7 \\
\hline 11 & & Gabbrovy & PR-1/234 & 8.3 & 1.12 & 2.93 & 1.41 & 1.24 & 3.9 & 0.704887 & 2.2 \\
\hline 12 & & & PR-1/266 & 7.9 & 1.29 & 2.94 & 1.43 & 1.28 & 5.31 & 0.705206 & 2.1 \\
\hline \multirow[t]{2}{*}{13} & & Norilsk area & $\begin{array}{c}n=8[67] \\
\text { MP-2b }\end{array}$ & & & & & & & 0.707640 & 1.7 \\
\hline & Norilsk & Norilsk area & [67] & & & & & & & & \\
\hline 14 & & Talnakh & $\begin{array}{c}n=6 \\
\text { OUG-2 }\end{array}$ & & & & & & & 0.7071238 & 0.8 \\
\hline 15 & & Norilsk 1 & $\begin{array}{l}n=9 \\
\mathrm{MN}-2\end{array}$ & & & & & & & 0.7067533 & 1.4 \\
\hline 16 & Gudchikhinsky & Norilsk area & $\begin{array}{c}n=1.75 / 2 \\
{[51]} \\
n=5\end{array}$ & 10.4 & 1.66 & 2.36 & 1.49 & 2.00 & 1.84 & 0.705435 & 3.9 \\
\hline 17 & & Norilsk area & $\begin{array}{c}\text { CY-50, } \\
X-51 / 130\end{array}$ & & & & & & & 0.705343 & 4.2 \\
\hline
\end{tabular}


The first rock variety, in fact, leucogabbro, formed oval or lenticular bodies (between few centimeters and few meters) with clear boundaries in gabbro-dolerites mostly in peripheral zones of ore-bearing intrusions or in their upper and lower parts. The mechanism of their formation was proposed by Likhachev [73,74] and suggests plagioclase accumulation and floatation during its early crystallization in magma when it rises to the surface in a conduit. In a chamber, magma pushes plagioclase mush into peripheral zones forming "inner leucogabbro". Sometimes this mush fills fractures near a chamber and forms "outer leucogabbro". Thus, Likhachev believes that "there was no significant break in the formation of these components of ore-bearing intrusions (i.e., leucogabbro and gabbro-dolerites of main intrusive body, N.K.), there is no doubt that they belong to a single magmatic system" (page 10 in [74]). Thus, inner leucogabbro bodies are not xenoliths (as it was written in [62]), because they are products of crystallization of the same magma. In this regard, external leucogabbro are apophyses of ore-bearing intrusions. The compositions of internal and external leucogabbro are identical in terms of the main components (Table 7).

Table 7. Weighted mean chemical compositions of the intrusions and leucogabbro (wt.\%) and their isotope characteristics.

\begin{tabular}{|c|c|c|c|c|c|c|c|c|}
\hline No. & 1 & 2 & 3 & 4 & 5 & 6 & 7 & 8 \\
\hline No Sample & MP-2b & SF-7 & ZF-37 & ZF-39 & 1 & 2 & 3 & 4 \\
\hline $\mathrm{SiO}_{2}$ & 47.63 & 48.86 & 48.45 & 49.64 & 48.53 & 48.22 & 47.97 & 49.51 \\
\hline $\mathrm{TiO}_{2}$ & 1.07 & 1.04 & 1.16 & 1.04 & 0.50 & 0.82 & 0.74 & 0.58 \\
\hline $\mathrm{Al}_{2} \mathrm{O}_{3}$ & 17.80 & 18.07 & 17.95 & 18.07 & 21.85 & 20.42 & 20.13 & 20.88 \\
\hline $\mathrm{Fe}_{2} \mathrm{O}_{3}$ & 12.21 & 10.88 & 14.11 & 13.42 & 7.81 & 9.76 & 9.68 & 7.34 \\
\hline $\mathrm{MnO}$ & 0.15 & 0.17 & 0.19 & 0.18 & 0.08 & 0.11 & 0.10 & 0.08 \\
\hline $\mathrm{MgO}$ & 6.68 & 6.05 & 6.40 & 7.89 & 5.64 & 4.76 & 5.54 & 4.68 \\
\hline $\mathrm{CaO}$ & 10.81 & 10.62 & 12.19 & 11.59 & 11.90 & 11.74 & 12.09 & 12.31 \\
\hline $\mathrm{Na}_{2} \mathrm{O}$ & 2.62 & 2.82 & 2.76 & 2.90 & 2.17 & 2.48 & 2.47 & 3.24 \\
\hline $\mathrm{K} 2 \mathrm{O}$ & 0.92 & 1.34 & 0.67 & 0.55 & 1.47 & 1.60 & 1.07 & 1.19 \\
\hline $\mathrm{P}_{2} \mathrm{O} 5$ & 0.11 & 0.15 & 0.13 & 0.11 & 0.05 & 0.09 & 0.21 & 0.19 \\
\hline $\mathrm{Cr}$ & 120 & 141 & 235 & 221 & & & & \\
\hline${ }^{87} \mathrm{Sr} /{ }^{86} \mathrm{Sr}$ & 0.707028 & & & & & & & \\
\hline$\varepsilon \mathrm{Nd}$ & 1.7 & & & & & & & \\
\hline No. & 9 & 10 & 11 & \multicolumn{2}{|c|}{12} & 13 & 14 & 15 \\
\hline No Sample & PR-1 & Katangsky 1 & Katangsky 2 & \multicolumn{2}{|c|}{ Kureysky (Khalil) } & Norilsk-Type & MD-27 & SF-10 \\
\hline $\mathrm{SiO}_{2}$ & 46.87 & 49.84 & 48.71 & \multicolumn{2}{|c|}{48.32} & 49.23 & 49.44 & 48.72 \\
\hline $\mathrm{TiO}_{2}$ & 1.33 & 1.44 & 1.52 & \multicolumn{2}{|c|}{1.59} & 1.00 & 0.90 & 0.89 \\
\hline $\mathrm{Al}_{2} \mathrm{O}_{3}$ & 14.96 & 14.59 & 15.07 & \multicolumn{2}{|c|}{14.30} & 15.32 & 16.53 & 14.68 \\
\hline $\mathrm{Fe}_{2} \mathrm{O}_{3}$ & 12.74 & 12.69 & 13.15 & \multicolumn{2}{|c|}{13.91} & 11.40 & 10.62 & 10.61 \\
\hline $\mathrm{MnO}$ & 0.19 & 0.18 & 0.19 & \multicolumn{2}{|c|}{0.20} & 0.17 & 0.15 & 0.16 \\
\hline $\mathrm{MgO}$ & 7.40 & 6.90 & 7.23 & \multicolumn{2}{|c|}{6.81} & 8.58 & 7.75 & 9.96 \\
\hline $\mathrm{CaO}$ & 9.81 & 10.11 & 9.97 & \multicolumn{2}{|c|}{10.41} & 11.85 & 12.03 & 10.96 \\
\hline $\mathrm{Na}_{2} \mathrm{O}$ & 2.60 & 3.27 & 3.42 & \multicolumn{2}{|c|}{3.53} & 2.08 & 3.52 & 2.19 \\
\hline $\mathrm{K}_{2} \mathrm{O}$ & 0.58 & 0.76 & 0.55 & \multicolumn{2}{|c|}{0.71} & 0.25 & 0.40 & 0.71 \\
\hline $\mathrm{P}_{2} \mathrm{O}_{5}$ & 0.19 & 0.14 & 0.17 & \multicolumn{2}{|c|}{0.21} & 0.09 & 0.24 & 0.12 \\
\hline $\mathrm{Cr}$ & 128 & 166 & 165 & \multicolumn{2}{|c|}{181} & 379 & 524 & 698 \\
\hline${ }^{87} \mathrm{Sr} /{ }^{86} \mathrm{Sr}$ & 0.705105 & & & \multicolumn{2}{|c|}{0.705694} & 0.705275 & & \\
\hline$\varepsilon \mathrm{Nd}$ & 2.01 & & & \multicolumn{2}{|c|}{ 2. 34} & 1.01 & & \\
\hline
\end{tabular}

Note: Nos. 1-4, Kruglogorsky subcomplex, Norilsk area, after [62]; 5-8, leucogabbro (5, 6, Norilsk 1 inner, 6 outer; 7 , Mount Chenraya, outer; 8, Talnakh, inner), after [72]; 9-13, intrusions of the Kulyumber river valley (9, Gabbrovy; 10 Katangsky complex, Intrusion 1 in Figure 3, Kulyumber site; 11, Katangsky complex, Intrusion 2 in Figure 3; 12, Kureysky complex, Khalil intrusion, Khalil site [51]; 13, Norilsk-type intrusion, Khalil site [51]); 14-15, Norilsk complex, Norilsk area (14 Norilsk 1, 15 Talnakh).

A similar idea on the origin of the Kruglogorsky leucogabbro belongs to Rad'ko [72], who assumes that the occurrence of the Kruglogorsky intrusions in any places is evidence to the location of ore-bearing intrusions nearby. On the basis of this suggestion, the Kruglogorsky sills can be used for prospecting new $\mathrm{Cu}-\mathrm{Ni}$ deposits. This approach formed a special geological method, which has been used by Norilskgeology Ltd. within the Kulyumber river valley. In this regard, the Gabbrovy intrusion is an indicator of closely located mineralization.

The second variety of leucogabbro with ataxitic structure formed horizons in the lower and upper zones of intrusions. They had a large thickness and comprised economic PGE-Cu-Ni mineralization 
in the ore-bearing intrusions (Talnakh, Norilsk 1). Their origin has been under discussion for many years $[75,77,78]$.

Meanwhile, the geological mapping and exploration carried out in the 1960s and 1970s, demonstrated that the outer leucogabbro formed separate intrusive bodies [53]. Although the composition and structure of the Gabbrovy intrusion were similar to those of the Kruglogorsky sills, there are several differences with them. It is enriched in $\mathrm{TiO}_{2}$ and $\mathrm{MgO}$ and depleted in $\mathrm{Al}_{2} \mathrm{O}_{3}$ in comparison with the leucogabbro sills of the Norilsk region $\left(1.35\right.$ and $1.05 \mathrm{TiO}_{2}, 7.6$ and $6.7 \mathrm{wt} . \% \mathrm{MgO}, 14$ and $17-18 \mathrm{wt} . \%$ $\mathrm{Al}_{2} \mathrm{O}_{3}$, respectively) and has different weighted mean composition and isotopic characteristics (Table 6). In fact, the Gabbrovy intrusion takes an intermediate position between the Kruglogorsky subcomplex and Katangsky complex. We guess that it is possible to regard it within the latter as a weakly differentiated body.

In the case of the Kruglogorsky subcomplex sills in the Norilsk region, it was obvious that they were formed from magmas differing in chemical composition from magmas of ore-bearing intrusions. Their parental magmas were more leucocratic (5-6\% wt. $\% \mathrm{MgO}$ in comparison with $10-12 \mathrm{wt} . \% \mathrm{MgO}$ for the Norilsk ore-bearing intrusions) and had higher $\varepsilon \mathrm{Nd}$ and lower $\mathrm{Sr}$ (Table 6). These features cannot be explained by the operation of fluids, as suggested in article [62]. The authors did not give any data on $\mathrm{H}_{2} \mathrm{O}, \mathrm{Cl}$, or $\mathrm{F}$ contents in magmas that could support this theory. Furthermore, the occurrence of amygdales in leucogabbro does not account for the enrichment of these rocks by volatile components because amygdales are typical of any rocks in the upper parts of subvolcanic intrusive bodies, not only in the Norilsk region but around the Siberian trap province.

The chemical compositions of rock-forming minerals from the studied intrusive complexes were very close to each other. In general, they reflected the composition of rocks, in particular, their magnesium number. The most significant mineral was olivine, since it is usually an early liquidus phase in rocks. Olivine varied significantly in differentiated intrusions. This has been established in numerous studies for the Norilsk ore-bearing intrusion, where its composition changes from 40 to 82 [24-26,43]. Most of the magnesium olivine compositions are typical of the picritic gabbro-dolerites and troctolites, in particular, for these rocks from the Norilsk 1 massif, studied by us in the core of the DM-27 borehole (Figures 14 and 15), where the forsterite component reached 80 mol.\%. These olivines were similar to olivines from the Intrusive 2 of the Kureysky complex, changing from $\mathrm{Fo}_{38}$ to $\mathrm{Fo}_{75}$ (Table $\mathrm{S} 1$ ). Meanwhile, the Norilsk-type intrusion in the Khalil site, attributed preliminarily to the Norilsk complex, contained very ferruginous olivine $\mathrm{Fo}_{31-46}$. Although the olivines from the Norilsk 1 intrusion were enriched in $\mathrm{NiO}$ (especially those from the picrite gabbro-dolerites containing sulfide ores), the olivines from the Intrusion 2 did not differ from them: They were even more nickel-rich at the same Fo. The minimum NiO occurred in the rocks from the Dzhatulsky massif and the trachydolerites of the Ergalakhsky complex. Most of the compositions of studied pyroxenes (Figure 16) almost all fell into the field of magnesium-rich augite and their points formed a small field in the En-Wo-Fs diagram. As in the case of olivine, pyroxenes from Intrusive 2 of the Kureysky complex were characterized by the greatest dispersion of compositions. 


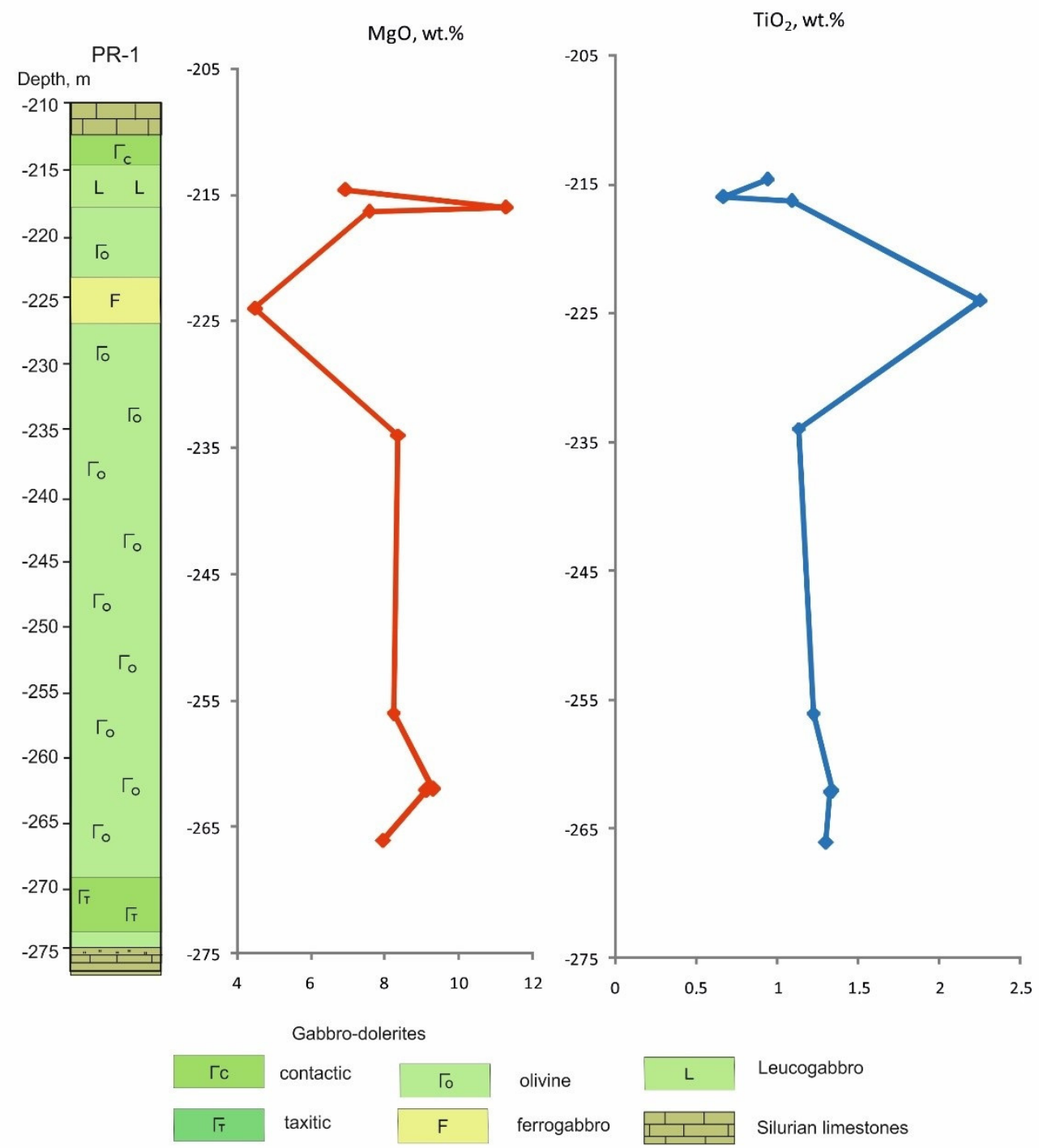

Figure 14. Inner structure of the Gabbrovy intrusion.

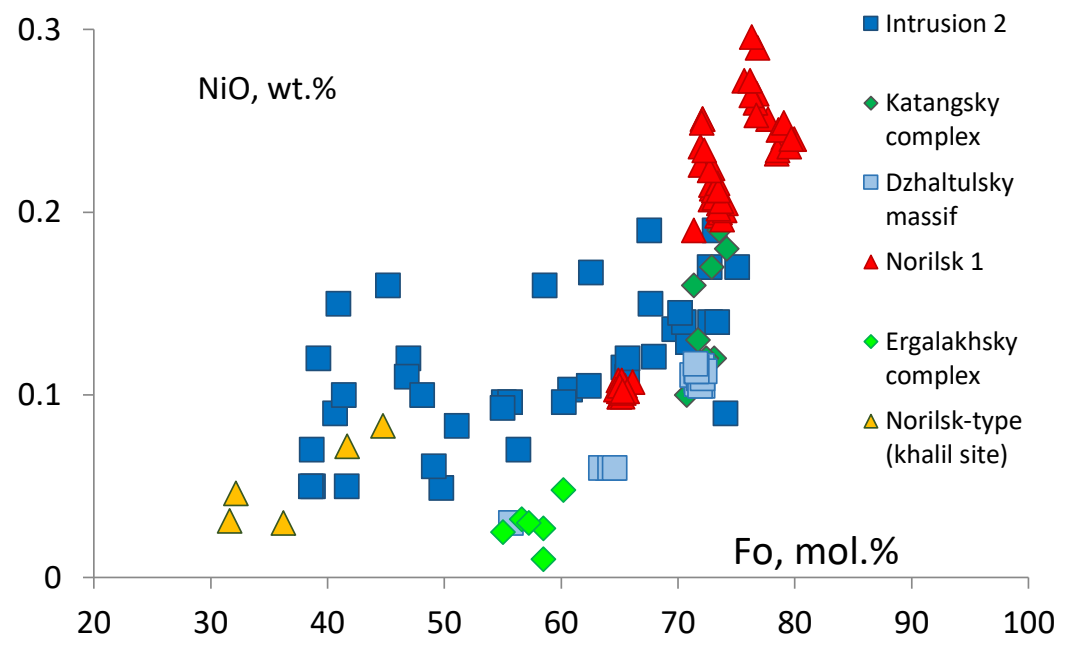

Figure 15. Diagram Fo-NiO for olivines from intrusive rocks. 


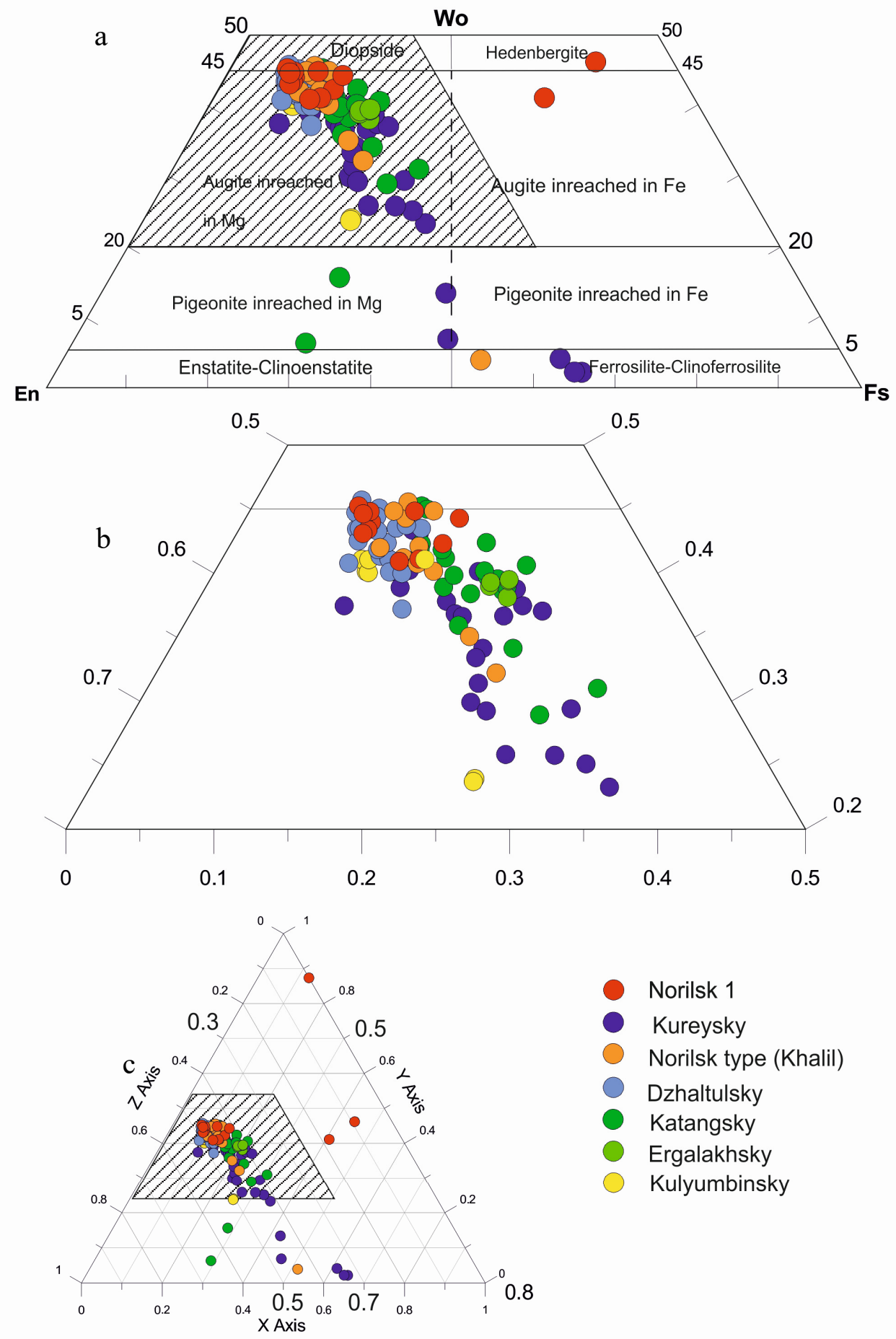

Figure 16. Pyroxenes' compositions from the intrusive rocks in diagram En-Wo-Fs, (a) trapezium with fields of pyroxens' compositions of the Kulyumber river valley, Norilsk 1, and Dzhaltulsky massifs; (b) the most widespread pyroxene compositions; (c) position of trapezium in diagram En-Wo-Fs.

\subsection{Magma Sources}

It should be assumed that the four groups of rocks mentioned above have various magma sources. The problem of the magma sources diversity has been considered for the Siberian platform for many years. Initially it was believed [1] that the diversity of all rocks in the province was due to the result 
of crystallization differentiation of a single magma. By the end of the 1980s, the diversity of rock compositions was explained by the fractionation of the primary picritic magma, resulting from the assimilation of crustal rocks and the influence of mantle fluids on the initial melt [5]. New geochemical data, including a distribution of rare elements in magmatic rocks and their isotopic characteristics, led to a new interpretation of the origin of igneous rocks in the Siberian platform. They were regarded as a product of crust contamination (up to $25-30 \%$ ) by primary mantle magma [35,36]. Later, the formation of different rocks was explained by simultaneous plume melting of at least two different lithospheric substrates $[79,80]$ or as a result of the action of two different plumes [41]. The variety of rocks was also explained by the different amount of pyroxenite component in the source, formed as a result of the interaction of peridotite with the substance of recycled oceanic crust [81].

The most contrasting compositions of rocks formed at different depths and located in the same place represent tholeiitic basalts and kimberlites that are believed to have been formed from multilevel conjugated sources [82].

The difference in sources at various depths was explained by an action of fluids as well [83]. Results of nonisothermal equilibrium physicochemical dynamics modeling show the occurrence of the following sequence of zones in over-asthenosphere continental mantle: (1) a zone where initial rocks were intensively sublimated and depleted by most petrogenic components; the restite in this case becomes carbonated, salinated, and graphitized; (2) a zone of $\mathrm{Si}$ and Fe enrichment and carbon deposition in initial rocks depleted in $\mathrm{Na}, \mathrm{K}, \mathrm{P}$, and $\mathrm{Mn}$; (3) a zone of diamond-bearing lherzolites enriched with Na; (4) a zone of hydrated rocks enriched with $\mathrm{K}$; (5) a zone of hydrated rocks not enriched with petrogenic components. Zone 2 can be responsible for the formation of kimberlite melts, zones 3 and 4 can be substrates of alkaline magma melting, and zone 5 can be the source of mafic tholeiitic magma. Unfortunately, amount of fluids was not estimated and their role in magma origin is disputable.

So, the spatial combination of rocks formed from different deep sources in the same blocks of crust remains completely unclear. We believe that the complex structure of the northwestern zone of the Siberian platform assumed the presence of faults of different depth which are the conduits for mantle and crustal magmas forming different igneous rocks.

On the basis of the geochemical and mineralogical data on igneous rocks at the Kulyumber river valley given in both parts of the article (Part I and Part II) we came to the following conclusions.

\section{Conclusions}

(1) The geochemical study of the volcanic rocks in the Kulyumber river valley allowed distinguishing the following formations similar to those of the Norilsk area: Syverminsky, Gudchikhinsky, Khakanchansky, and Nadezhdinsky. The absence of the Ivakinsky formation in the studied area implied that it pinched out from the north to the south. The tuffs of the Syverminsky formation were analyzed for the first time, and we have demonstrated how they differ from the Khakanchansky tuffs. Basalts of the Gudchikhinsky formation from the Kulyumber area were enriched in alkalis and depleted in $\mathrm{U}$ and Th, in comparison with the Gudchikhinsky basalts from the Norilsk area.

(2) The studied intrusive rocks could be subdivided into three groups on the basis of major and trace elements' contents: (1) Subalkaline rocks ( $\mathrm{MgO}=4 \mathrm{wt} . \%, \mathrm{TiO}_{2}=3.3 \mathrm{wt} . \%$ ) with $(\mathrm{La} / \mathrm{Sm}) \mathrm{n}=2.5-2.8$ and $\mathrm{Gd} / \mathrm{Yb}) \mathrm{n}=1.9-2.1$ (Ergalakhsky complex); (2) mafic rocks $\left(\mathrm{MgO}=6-9 \mathrm{wt} . \%, \mathrm{TiO}_{2}=0.8-1.7 \mathrm{wt} . \%\right)$ with strong negative $\mathrm{Ta}-\mathrm{Nb}$ and positive $\mathrm{Pb}$ anomalies and varying ratios $(\mathrm{La} / \mathrm{Sm}) \mathrm{n}=1.3-1.0,(\mathrm{Gd} / \mathrm{Yb}) \mathrm{n}=1.2-1.5$ (Katangsky, Kureysky, and Norilsk complexes, Kruglogorsky subcomplex); and (3) mafic rocks ( $\left.\mathrm{MgO}=6-7 \mathrm{wt} . \%, \mathrm{TiO}_{2}=1.4 \mathrm{wt} . \%\right)$ without $\mathrm{Ta}-\mathrm{Nb}$ and $\mathrm{Pb}$ anomalies with moderate $(\mathrm{La} / \mathrm{Sm}) \mathrm{n}=2.00$ and high $(\mathrm{Gd} / \mathrm{Yb}) \mathrm{n}=2.0$ ratios. The first two groups were similar to the rocks from the Norilsk area and Tunguska syneclese, while the rocks of the third group were unknown earlier in the NW Siberian platform and have been attributed by the authors to a new intrusive complex named Kulyumbinsky complex. 
(3) Intrusive rocks of the second group formed massifs ranging in composition. The Norilsk complex was characterized by strong rock differentiation and elevated $\mathrm{MgO}$ and $\mathrm{Cr}$, while Katangsky and Kureysky complexes of the Kulyumber river valley could not be distinguished from one another on the basis of geochemical data. A novel type of intrusion was recognized in the Khalil site. It takes an intermediate position between Norilsk and Katangsky complexes due to low $\mathrm{TiO}_{2}$ and elevated $\mathrm{Cr}$ contents. Intrusions of the Daldykansky and Ogonersky complexes have not been found in the Kukyumber river valley.

(4) Igneous rocks of the Kulyumber river valley originated from different sources: Mantle and crustal. The products of the first are the volcanic rocks of the Gudchikhinsky formation $\left({ }^{87} \mathrm{Sr} /{ }^{86} \mathrm{Sr}=0.706034\right.$ and $\left.\varepsilon \mathrm{Nd}=4.0\right)$ and gabbro-dolerites of the Kulyumbinsky complex $\left({ }^{86} \mathrm{Sr} /{ }^{87} \mathrm{Sr}=0.70675\right.$ and $\left.\varepsilon \mathrm{Nd}=-1.0\right)$. A separate source is typical of the Ergalakhsky trachydolerites $\left({ }^{87} \mathrm{Sr} /{ }^{86} \mathrm{Sr}=0.706034\right.$ and $\left.\varepsilon \mathrm{Nd}=-3.8\right)$, and an another source is typical of the rocks of many complexes (Norilsk, Katangsky, Kureysky, ${ }^{87} \mathrm{Sr} /{ }^{86} \mathrm{Sr}=0.70489-0.70702$, and $\varepsilon \mathrm{Nd}=0.4-2.2$, respectively).

(5) The diversity of igneous rocks within the Kulyumber river valley could be explained by the complex tectonic structure of the paleorift zone, which is characterized by a long tectonic evolution and the effect of deep faults.

Supplementary Materials: The following are available online at http://www.mdpi.com/2075-163X/10/5/415/s1, Table S1: Composition of rock-forming minerals, wt.\%.

Author Contributions: Conceptualization N.K., methodology B.B. and A.D.; investigation, B.G., A.L., formal analyses T.B.B. All authors have read and agreed to the published version of the manuscript.

Funding: This study was financially supported by the Russian Foundation for Basic Research, projects Nos. 18-05-70094 and 19-05-00654.

Acknowledgments: We are grateful to I. Sidorenko and M. Nesterenko for their help in the field trip and geologists of Norilskgeolgy Ltd. for the permission for cores' sampling. The authors thank N. Kononkova and V. Taskaev for microprobe analyses and N. Svirskaya and V. Turkov for samples' preparation.

Conflicts of Interest: The authors declare no conflict of interest.

\section{References}

1. Sobolev, V.S. Petrology of Traps from the Siberian Platform; Proceedings Arctic Insitiute: Leningrad, Russia, 1936; Volume 43, p. 224. (In Russian)

2. Malitch, N.S. Tectonic Development of the Cover of the Siberian Platform; Nedra: Moscow, Russia, 1975; p. 215. (In Russian)

3. Zolotukhin, V.V.; Vasil'ev, Y.R.; Dyuzhikov, O.A. Diversity of Traps and Initial Magmas: A Case of the Siberian Platform; Nauka: Novosibirsk, Russia, 1978; p. 289. (In Russian)

4. Masaitis, V.L. Permian and Triassic volcanism of Siberia. Zap. Vmo 1983, 4, 412-425. (In Russian)

5. Zolotukhin, V.V.; Vilensky, A.M.; Dyuzhikov, O.A. Basalts of the Siberian Platform; Nauka: Novosibirsk, Russia, 1986; p. 245. (In Russian)

6. Zolotukhin, V.V.; Al'mukhamedov, A.I. Traps of the Siberian Platform. In Continental Flood Basalts; Macdougall, J.D., Ed.; Kluwer Academic Publishers: Dordrecht, The Netherlands, 1988; pp. 273-310.

7. Zolotukhin, V.V.; Al'mukhamedov, A.I. Basalts of the Siberian platform: Composition and mechanism of formation. In Traps of Siberia and Deccan: Similarities and Differences; Nauka: Novosibirsk, Russia, 1991; pp. 7-39. (In Russian)

8. Oleynikov, B.V.; Tomshin, M.D. Evolution of basic intrusive magmatism of the Siberian platform in time. In Traps of Siberia and Deccan: Similarities and differences; Nauka: Novosibirsk, Russia, 1991; pp. 39-63. (In Russian)

9. Renne, P.R.; Basu, A.R. Rapid eruption of the Siberian Traps flood basalts at the Permo-Triassic boundary. Science 1991, 253, 176-179. [CrossRef]

10. Sharma, M.; Basu, A.R.; Nesterenko, G.V. Nd-Sr isotopes, petrochemistry, and origin of the Siberian flood basalts, Siberia. Geochim. Cosmochim. Acta 1991, 55, 1183-1192. [CrossRef] 
11. Sharma, M.; Basu, A.R.; Nesterenko, G.V. Temporal Sr-, Nd-, and Pb-isotopic variations in the Siberian flood basalts: Implications for the plume-source characteristics. Earth Planet. Sci. Lett. 1992, 113, 365-381. [CrossRef]

12. Coffin, M.F.; Eldholm, O. Large igneous provinces. Crustal structure, dimensions and external consequences. Rev. Geoghys. 1994, 32, 1-36. [CrossRef]

13. Reichow, M.K.; Saunders, A.D.; White, R.V.; Pringle, M.S.; Al'Mukhamedov, A.I.; Medvedev, A.I.; Kirda, N.P. ${ }^{40} \mathrm{Ar} /{ }^{39} \mathrm{Ar}$ dates from the West Siberian Basin: Siberian flood basalt province doubled. Science 2002, 296, 1846-1849. [CrossRef]

14. Al'mukhamedov, A.I.; Medvedev, A.Y.; Zolotukhin, V.V. Evolutions of Permo-Triassic basalts of the Siberian Platform in time and space. Petrology 2004, 12, 339-353.

15. Dobretsov, N.L.; Borisenko, A.S.; Izokh, A.E.; Zhmodik, S.M. Termochemical model of Permo-Triassic plume of Euroausia as a basement for genesis understanding and prospecting of copper-nickel, noble- and rare metals deposits. Rus. Geol. Geophys. 2010, 51, 1159-1180. [CrossRef]

16. Ernst, R.E. Large Igneous Provinces; Cambridge University Press: Cambridge, UK, 2014; 653p.

17. Schmidt, A.; Fristad, K.; Elkins-Tanton, L. (Eds.) Volcanism and Global Environmental Change; Special Volume; Cambridge University Press: Cambridge, UK, 2015.

18. Godlevsky, M.N. Traps and Ore-Bearing Intrusion; Gosgeoltekhizdat: Moscow, Russia, 1959; 61p. (In Russian)

19. Zolotukhin, V.V.; Ryabov, V.V.; Vasil'ev, Y.R.; Shatkov, V.A.; Шатков, B.A. Petrology of the Talnakh Differentiated Ore-Bearing Trap Intrusion; Nauka: Novosibirsk, Russia, 1975; 243p. (In Russian)

20. Genkin, A.D.; Distler, V.V.; Gladyshev, G.D. Sulfide Copper-Nickel Ores of the Norilsk Deposits; Nauka: Moscow, Russia, 1981; 295p. (In Russian)

21. Dyuzhikov, O.A.; Distler, V.V.; Strunin, B.M.; Mkrtychyan, A.K.; Sherman, M.L.; Sluzhenikin, S.F.; Lurye, A.M. Geology and Ore Potential of the Noril'sk Ore District; Nauka: Moscow, Russia, 1988. (In Russian)

22. Distler, V.V.; Grokhovskaya, T.L.; Evstigneeva, T.L.; Sluzhenikin, S.F.; Filimonova, A.A.; Dyuzhikov, O.A. Petrology of Magmatic Sulfide ore Formation; Nauka: Moscow, Russia, 1988; p. 232. (In Russian)

23. Naldrett, A.J. A model for the Ni-Cu-PGE ores of the Noril'sk region and its application to other areas of flood basalts. Econ. Geol. 1992, 87, 1945-1962. [CrossRef]

24. Ryabov, V.V. Olivines and Their Petrological Significance; Nauka: Novosibirsk, Russia, 1992. (In Russian)

25. Ryabov, V.V.; Shevko, A.Y.; Gora, M.P. Trap Magmatism and Ore Formation in the Siberian Noril'sk Region; Springer: Amsterdam, The Netherlands, 2014; Volume 1.2.

26. Likhachev, A.P. Kharaelakh intrusion and its Pt-Cu-Ni ores. Rudy I Met. 1998, 3, 48-62. (In Russian)

27. Likhachev, A.P. Platinum-Copper-Nickel and Platinum Deposits; Eslan: Moscow, Russia, 2006; p. 496. (In Russian)

28. Malitch, K.N.; Belousova, E.A.; Griffin, W.L.; Badanina, I.Y.; Latypov, R.M.; Sluzhenikin, S.F. Chapter 7. New insights on the origin of ultramafic-mafic intrusions and associated Ni-Cu-PGE sulfide deposits of the Noril'sk and Taimyr provinces, Russia: Evidence from radiogenic and stable isotope data. In Processes and ore Deposits of Ultramafic-Mafic Magmas through Space and Time; Elsevier: Chennai, India, 2018; pp. 197-238.

29. Krivolutskaya, N.; Gongalsky, B.; Kedrovskaya, T.; Kubrakova, I.; Tyutyunnik, O.; Chikatueva, V.; Bychkova, Y.; Kovalchuk, E.; Kononkova, N.; Yakushev, A. Geology of the Western Flanks of the Oktyabr'skoe Deposit, Noril'sk District, Russia: Evidence of a Closed Magmatic System. Minealium Depos. 2019, 54, 611-630. [CrossRef]

30. Basu, A.R.; Poreda, R.J.; Renne, P.R.; Teichmann, F.; Vasil'ev, Y.R.; Sobolev, N.V.; Turrin, B.D. High-3He plume origin and temporal-spatial evolution of the Siberian flood basalts. Science 1995, 269, 822-825. [CrossRef]

31. Dobretsov, N.L.; Borisenko, A.S.; Izokh, A.E. Termochemical deep mantle plums as a source of ore potencial on the planet. Nauka Iz Peroykh Ruk 2011, 6, 37-43. (In Russian)

32. Sobolev, S.V.; Sobolev, A.V.; Kuzmin, D.V.; Krivolutskaya, N.A.; Arndt, N.T.; Radko, V.A.; Petrunin, A.G.; Vasilev, Y.R. Linking mantle plumes, large igneous provinces and environment catastrophes. Nature 2011, 477, 312-316. [CrossRef]

33. Volkov, I.D. Distribution of trace elements in igneous rocks of the Norilsk region. In Problems of Magmatism, Metamorphism, and Mineralization; Geoltekhizdat: Moscow, Russia, 1963; pp. 29-38.

34. Balashov, Y.A.; Nesterenko, G.V. Distribution of trace elements in traps of the Siberian platform. Geochem. Int. 1966, 7, 854-860. 
35. Lightfoot, P.C.; Hawkesworth, C.J.; Hergt, J.; Naldrett, A.J.; Gorbachev, N.S.; Fedorenko, V.A. Remobilisation of the continental lithosphere by a mantle plume: Major-, trace-element, and $\mathrm{Sr}-, \mathrm{Nd}-$, and Pb-isotopic evidence from picritic and tholeiitic lavas of the Noril'sk District, Siberian Trap, Russia. Contrib. Miner. Pet. 1993, 114, 171-188. [CrossRef]

36. Wooden, J.L.; Czamanske, G.K.; Fedorenko, V.A.; Arndt, N.T.; Chauvel, C.; Bouse, R.M.; King, B.S.; Knight, R.J.; Siems, D.F. Isotopic and trace-element constraints on mantle and crustal contributions to Siberian continental flood basalts, Norilsk area, Siberia. Geochim. Cosmochim. Acta 1993, 57, 3677-3704. [CrossRef]

37. Arndt, N.; Lehnert, K.; Vasil'ev, Y. Meimechites: Highly magnesian lithosphere-contaminated alkaline magmas from deep subcontinental mantle. Lithos 1995, 34, 41-59. [CrossRef]

38. Dalrymple, B.G.; Czamanske, G.K.; Fedorenko, A.; Simonov, O.N.; Lanphere, M.A.; Likhachev, A.P. A reconnaisance ${ }^{40} \mathrm{Ar} /{ }^{39} \mathrm{Ar}$ geochronological study of ore-bearing and related rocks, Siberian Russia. Geochim. Cosmochim. Acta 1995, 59, 2071-2083. [CrossRef]

39. Vasil'ev, Y.R.; Zolotukhin, V.V. The Maimecha-Kotuy alkaline-ultramafic province of the northern Siberian platform, Russia. Episodes 1995, 18, 155-164.

40. Fedorenko, V.A.; Lightfoot, P.C.; Naldrett, A.J.; Czamanske, G.K.; Hawkesworth, C.J.; Wooden, J.L.; Ebel, D.S. Petrogenesis of the Siberian flood-basalt sequence at Noril'sk. Int. Geol. Rev. 1996, 38, 99-135. [CrossRef]

41. Arndt, N.; Chauvel, C.; Czamanske, G.; Fedorenko, V. Two mantle sources, two plumbing systems: Tholeiitic and alkaline magmatism of the Maymecha River basin, Siberian flood volcanic province. Contrib. Miner. Pet. 1998, 133, 297-313. [CrossRef]

42. Sobolev, A.V.; Krivolutskaya, N.A.; Kuzmin, D.V. Petrology of the Parental Melts and Mantle Sources of Siberian Trap Magmatism. Petrology 2009, 17, 253-286. [CrossRef]

43. Krivolutskaya, N.A. Siberian Traps and Pt-Cu-Ni Deposits in the Noril'sk Area; Springer: Amsterdam, The Netherlands, 2016; p. 361.

44. Naldrett, A.J.; Lightfoot, P.C.; Fedorenko, V.; Doherty, W.; Gorbachev, N.S. Geology and geochemistry of intrusions and flood basalts of the Noril'sk Region, USSR, with applications for the origin of the Ni-Cu ores. Econ. Geol. 1992, 87, 975-1004. [CrossRef]

45. Lul'ko, V.A.; Fedorenko, V.A.; Distler, V.V.; Sluzhenikin, S.F.; Kunilov, V.E.; Stekhin, A.I.; Ryabikin, V.A.; Simonov, O.N.; Zen'ko, T.E. Geology and Ore Deposits of the Noril'sk Region; Guidebook VII; IPS: Moscow, Russia, 1994; p. 67.

46. Naldrett, A.J.; Fedorenko, A.; Lightfoot, P.C.; Kunilov, E.; Gorbachev, N.S.; Doherty, W.; Johan, Z. Ni-Cu-PGE deposits of the Noril'sk region, Siberia: Their formation in conduits for flood basalt volcanism. Trans. Inst. Min. Metall. 1995, 104, B18-B36.

47. Arndt, N.T.; Czamanske, G.K.; Walker, R.J.; Chauvel, C.; Fedorenko, V.A. Geochemistry and origin of the intrusive hosts of the Noril'sk-Talnakh Cu-Ni-PGE sulfide deposits. Econ. Geol. 2003, 98, 495-515. [CrossRef]

48. Malitch, K.N.; Badanina, I.Y.; Tuganova, E.V. Ore-Bearing Ultramafic-Mafic Intrusions of Polar Siberia: New Insight on the Age, Origin and Predicted Criteria; IGG UB RAS: Ekaterinburg, Russia, 2018; 287p. (In Russian)

49. Krivolutskaya, N.; Latyshev, A.; Dolgal, A.; Gongalsky, B.; Makareva, E.; Makarev, A.; Svirskaya, N.; Bychkova, Y.; Yakushev, A.; Asavin, A. Unique PGE-Cu-Ni Noril'sk Deposits, Siberian Trap Province: Magmatic and Tectonic Factors in Their Origin. Minerals 2019, 9, 66. [CrossRef]

50. State Geological Map of Russian Federation. 1:1,000,000 Scale (New Version); Explanatory Note 2000, St-Pet.; VSEGEI: Norilsk, Russia, 2000; 479p.

51. Krivolutskaya, N.; Belyatsky, B.; Gongalsky, B.; Dolgal, A.; Lapkovsky, A.; Malitch, K.; Taskaev, V.; Svirskaya, N. Geochemistry of magmatic rocks in the northwestern Siberian Trap Province, Kulyumber river valley. Part I: Rocks of the Khalil area. Minerals 2020, 10, 409. [CrossRef]

52. Legend for 1:50,000 Scale Map, Noril'sk Group; Lyul'ko, V.A. (Ed.) Geoinformmark: Moscow, Russia, $1993 ;$ p. 53. (In Russian)

53. Sherman, M.L. (Ed.) Geological Map of the Noril'sk Ore Dstrict,1:200000 Scale; VSEGEI: Leningrad, Russia, 1991.

54. Krivolutskaya, N.; Kedrovskaya, T. Structure and composition of the Nadayansky lava flow: An example of homogeneity of lava flows of the Siberian trap province. Geochem. Int. 2020, 58, 363-376. [CrossRef]

55. Karandashev, V.K.; Khvostikov, V.A.; Nosenko, S.V.; Burmii, Z. Stable highly enriched isotopes in routine analyses of rocks, soils, grounds, and sediments by ICP-MS. Inorg. Mater. 2017, 53, 1432-1441. [CrossRef]

56. Stacey, J.S.; Kramers, J.D. Approximation of terrestrial lead isotope evolution by a two-stage model. Earth Planet. Sci. Lett. 1975, 26, 207-221. [CrossRef] 
57. Geological Map of the Norilsk Region and Adjacent Territories, R-45-XXIII, XXIV, Q-45; Explanatory Note; Datsenko, V.A.; Markov, F.B. (Eds.) Nedra: Moscow, Russia, 1969; 81p. (In Russian)

58. Hofmann, A.W. Chemical differentiation of the earth: The relationship between mantle, continental and ocean crust. Earth Planet Sci. Lett. 1988, 90, 297-314. [CrossRef]

59. Pavlov, V.E.; Galley, I. Upper Cambrian to Middle Ordovician magnitostratigraphy from the Kulyumbe river section (northwestern Siberia). Phys. Earth Planet. Int. 1998, 108, 49-59. [CrossRef]

60. Latyshev, A.V.; Krivolutskaya, N.A.; Ulyahina, P.S.; Bychkova, Y.V.; Gongalsky, B.I. Intrusions of the Kulyumber river valley, NW Siberian traps province: Paleomagnetism, magnetic fabric and geochemistry. In Recent Advances in Rock Magnetism, Environmental Magnetism and Paleomagnetism; Springer: Amsterdam, The Netherlands, 2018; pp. 67-83.

61. Krivolutskaya, N.A.; Sobolev, A.V.; Snisar, S.G.; Gongalskiy, B.I.; Hauff, B.; Kuzmin, D.V.; Tushentsova, I.N.; Svirskaya, N.M.; Kononkova, N.N.; Schlychkova, T.B. Mineralogy, geochemistry and stratigraphy of the Maslovsky Pt-Cu-Ni sulfide deposit, Noril'sk Region, Russia: Implications for relationship of ore-bearing intrusions and lavas. Miner. Depos. 2012, 47, 69-88. [CrossRef]

62. Sluzhenikin, S.F.; Malitch, K.N.; Grigorieva, A.V. Differentiated mafic-ultramafic intrusions of the Kruglogorsky type in the Norilsk area: Petrology and ore potential. Petrology 2018, 26, 280-313. [CrossRef]

63. Sluzhenikin, S.F.; Krivolutskaya, N.A. Pyasino-Vologochan intrusion: Geological structure and platinum-copper-nickel ores (Norilsk region). Geol. Ore Depos. 2015, 57, 424-444. [CrossRef]

64. Le Maitre, R.W.; Streckeisen, A.; Zanettin, B.; Le Bas, M.J.; Bonin, B.; Bateman, P.; Belieni, G.; Dudek, A.; Efremova, S.; Keller, J.; et al. Igneous Rocks: A Classification and Glossary of Terms; Le Maitre, R.W., Ed.; Cambridge University Press: Cambridge, UK, 2002; p. 37.

65. Krivolutskaya, N.A. Formation of PGE-Cu-Ni deposits in the process of evolution of flood basalt magmatism in the Noril'sk region. Geol. Ore Depos. 2011, 53, 303-339. [CrossRef]

66. Dneprovskaya, M.B.; Frenlel, M.Y.; Yaroshevsky, A.A. Quantitative 'model of layering of the Talnakh intrusion. In Constrants of Models for Ore-Forming Systems; Nauka: Novosibirsk, Russia, 1987; pp. 96-106. (In Russian)

67. Petrov, O.V. (Ed.) Isotope Geology of the Norilsk Deposits; VSEGEI: St-Peterburg, Russia, 2017; 348p. (In Russian)

68. Sudbury-Norils Symposium; Geological Survey: Ottawa, ON, Canada, 1994; Volume 5, 390p.

69. Sereda, E.; Belyatsky, B.; Krivolutskaya, N. Geochemistry and Geochronology of Southern Norilsk Intrusions, SW Siberian Traps. Minerals 2020, 10, 165. [CrossRef]

70. Zolotukhin, V.V.; Vilensky, A.M.; Vasil'ev, Y.R.; Mezhvilk, A.A.; Ryabov, V.V.; Stsherbakova, Z.V. Magnesium Basites of Western Part of the Siberian Platform and Its Nickel Mineralization; Nauka: Novosibirsk, Russia, 1984; 208p. (In Russian)

71. Malitch, N.S.; Grinson, A.S.; Tuganova, E.V.; Chernyshev, N.M. Rifting of the Siberian platform. In Proceedings of the 28th session of International Geological Congress, Tectonic Processes, Moscow, Russia, 4-14 August 1984; Nauka: Moscow, Russia, 1988; pp. 184-193.

72. Rad'ko, V.A. Facies of Intrusive and Effusive Magmatism in the Norilsk Area; VSEGEI: St-Petersburg, Russia, 2016; 225p. (In Russian)

73. Likhachev, A.P. Role of leucocratic gabbro in formation of the Norilsk ore-bearing intrusions. Izv. Acad. Sci. 1965, 12, 50-66. (In Russian)

74. Likhachev, A.P. The possibility of selt-enrichment of the ore substance and the heavy sulfur isotope $\left({ }^{34} \mathrm{~S}\right)$ of the mantle magmas that form $\mathrm{Cu}-\mathrm{Pt}-\mathrm{Ni}$ deposits and a promising area for ore localization in the Norilsk region. Otechestvennaya Geol. 2019, 3, 1-18. (In Russian)

75. Zolotukhin, V.V. Mafic pegmatiodes of the Norilsk Ore-Bearing Intrusions and Origin of the Norilsk Ores; OIGG: Novosibirsk, Russia, 1997; 90p. (In Russian)

76. Dogin, D.A.; Batuev, B.N. Atlas of Textures and Structure of the Rocks from the Norilsk Area; Nedra: Leningrad, Russia, 1971; 468p. (In Russian)

77. Krivolutskaya, N.A.; Ariskin, A.A.; Sluzhenikin, S.F.; Turovtsev, D.M. Geochemical thermometry of rocks of the Talnakh intrusion: Assessment of the melt composition and the crystallinity of the parental magma. Petrology 2001, 9, 389-414.

78. Krivolutskaya, N.A.; Sobolev, A.V.; Mikhailov, V.N.; Plechova, A.A.; Kostitsyn, Y.A.; Roschina, I.A.; Fekiacova, Z. Parental melt of the Nadezhdinsky Formation: Geochemistry, petrology and connection with Cu-Ni deposits (Noril'sk area, Russia). Chem. Geol. 2012, 302-303, 87-105. [CrossRef] 
79. Sharma, M. Siberian Traps. In Large Igneous Provinces: Continental, Oceanic, and Planetary Flood Volcanis; Mahoney, J.J., Coffin, M.F., Eds.; American Geophysical Union: Wshington, DC, USA, 1997; Volume 100, pp. 273-295.

80. Basu, A.R.; Hannigan, R.E.; Jacobsen, S.B. Melting of the Siberian mantle plume. Geophys. Res. Lett. 1998, 25, 2209-2212. [CrossRef]

81. Sobolev, A.V.; Hofmann, A.W.; Kuzmin, D.V.; Yaxley, G.M.; Arndt, N.T.; Chung, S.L.; Danyushevsky, L.V.; Elliott, T.; Frey, F.A.; Garcia, M.O.; et al. The amount of Recycled Crust in Sources of Mantle-Derived Melts. Science 2007, 316, 412-417. [CrossRef]

82. Rotman, A.Y. Subalkaline Basites of Kimberlite Controlling structures in the Eastern Siberia; Abstract of Dissertation; IGM: Novosibirsk, Russia, 2002; 42p.

83. Sharapov, V.N.; Chudnenko, K.V.; Mazurov, M.P.; Perepechko, Y.V. Metasomatic zoning of subcratonic lithosphere in Siberia: Physicochemical modeling. Russ. Geol. Geophys. 2009, 50, 1107-1118. [CrossRef]

(C) 2020 by the authors. Licensee MDPI, Basel, Switzerland. This article is an open access article distributed under the terms and conditions of the Creative Commons Attribution (CC BY) license (http://creativecommons.org/licenses/by/4.0/). 